JOURNAL OF

SYMPLECTIC GEOMETRY

Volume 11, Number 2, 179-230, 2013

\title{
NEW TECHNIQUES FOR OBTAINING SCHUBERT-TYPE FORMULAS FOR HAMILTONIAN MANIFOLDS
}

\author{
Silvia Sabatini and Susan Tolman
}

In $[\mathbf{G T}]$, Goldin and Tolman extend some ideas from Schubert calculus to the more general setting of Hamiltonian torus actions on compact symplectic manifolds with isolated fixed points. (See also $[\mathrm{Kn99,Kn08].)} \mathrm{The} \mathrm{main} \mathrm{goal} \mathrm{of} \mathrm{this} \mathrm{paper} \mathrm{is} \mathrm{to} \mathrm{build} \mathrm{on} \mathrm{this} \mathrm{work} \mathrm{by}$ finding more effective formulas. More explicitly, given a generic component of the moment map, they define a canonical class $\alpha_{p}$ in the equivariant cohomology of the manifold $M$ for each fixed point $p \in M$. When they exist, canonical classes form a natural basis of the equivariant cohomology of $M$. In particular, when $M$ is a flag variety, these classes are the equivariant Schubert classes. It is a long-standing problem in combinatorics to find positive integral formulas for the equivariant structure constants associated to this basis. Since computing the restriction of the canonical classes to the fixed points determines these structure constants, it is important to find effective formulas for these restrictions. In this paper, we introduce new techniques for calculating the restrictions of a canonical class $\alpha_{p}$ to a fixed point $q$. Our formulas are nearly always simpler, in the sense that they count the contributions over fewer paths. Moreover, our formula is manifestly positive and integral in certain important special cases.

\section{Contents}

0. Introduction

1. Canonical classes

2. GKM spaces

3. The most general theorem

4. The Hamiltonian cone 
6. Positive integral formulas for Schubert classes

6.1. The canonical graph of a generic coadjoint orbit

6.2. Maps between coadjoint orbits

6.3. Generic coadjoint orbits of type $A_{n}$

\section{Introduction}

In $[\mathbf{G T}]$, Goldin and Tolman extend some ideas from Schubert calculus to the more general setting of Hamiltonian torus actions on compact symplectic manifolds with isolated fixed points. (Knutson found closely related formulas for the Duistermaat-Heckman measure in the algebraic case in [Kn99,Kn08].) Given a generic component of the moment map, they define a canonical class $\alpha_{p}$ in the equivariant cohomology of the manifold $M$ for each fixed point $p \in M$ (see Definition 0.1 below). When they exist, these canonical classes form a natural basis of the equivariant cohomology of $M$. In particular, when $M$ is a flag variety, these classes are the equivariant Schubert classes (see $[\mathbf{K u}]$ and Proposition 6.5). It is a long-standing problem in combinatorics to find positive integral formulas for the equivariant structure constants associated to this basis. Since computing the restriction of the canonical classes to the fixed points determines these structure constants and hence the (equivariant) cohomology ring of $M$, it is important to find effective formulas for these restrictions. Building on ideas of Guillemin and Zara $[\mathbf{G Z}]$, Goldin and Tolman show that the restriction of a canonical class $\alpha_{p}$ to a fixed point $q$ can be calculated by a rational function that only depends on the following information: the value of the moment map at fixed points, and the restriction of other canonical classes to points of index exactly two higher. Moreover, the restriction formula in $[\mathbf{G T}]$ is manifestly positive whenever the restrictions themselves are all positive, including when $M$ is a coadjoint orbit.

However, the results in $[\mathbf{G T}]$ differ from Schubert calculus in several important ways. For example, the individual summands in that formula are almost never integral; essentially, this only holds when $M$ is $\mathbb{C P}^{n}$. In contrast, in the combinatorics literature, a manifestly positive integral formula for the restriction of equivariant Schubert classes on $M=G / B$ is already known (see Appendix D.3 in [AJS, B]). The main goal of this paper is to bridge this gap by giving formulas which, like the formula in $[\mathbf{G T}]$, are valid in 
the much broader Hamiltonian category, but which are simpler in the sense that they count the contribution over fewer paths. Indeed, we want these contributions to be manifestly positive and integral whenever possible, and to understand geometrically when this occurs. This project was inspired by an early version of $[\mathbf{Z} \mathbf{a}]$, where $\mathrm{C}$. Zara used combinatorial tools to re-derive the integral formula in $[\mathbf{A J S}, \mathbf{B}]$ for the case of a coadjoint orbit of type $A_{n}$ from the formula in $[\mathbf{G T}]$, by taking limits as the cohomology class of the symplectic form varies.

Before giving more precise statements, let us define a few terms. Let $T$ be a (compact) torus with Lie algebra $\mathfrak{t}$ and lattice $\ell \subset \mathfrak{t}$, and $(\cdot, \cdot)$ the natural pairing between $\mathfrak{t}^{*}$ and $\mathfrak{t}$. Let $T$ act on a compact symplectic manifold $(M, \omega)$ with moment map $\psi: M \rightarrow \mathfrak{t}^{*}$. By definition,

$$
\iota_{X_{\xi}} \omega=-d \psi^{\xi} \quad \text { for all } \xi \in \mathfrak{t},
$$

where $X_{\xi}$ denotes the vector field on $M$ generated by the action and $\psi^{\xi}(x)=$ $(\psi(x), \xi)$. In this case, we say that the triple $(M, \omega, \psi)$ is a Hamiltonian T-manifold. Now, assume that $M$ has a discrete-fixed set and fix a generic $\xi \in \mathfrak{t}$, that is, assume that $(\eta, \xi) \neq 0$ for each weight $\eta \in \ell^{*} \subset \mathfrak{t}^{*}$ in the isotropy representation of $T$ on $T_{p} M$ for every fixed point $p$. Given a fixed point $p \in M^{T}$, let $\lambda(p)$ be the number of positive weights of the isotropy action on $T_{p} M$. Let $\Lambda_{p}^{-} \in \operatorname{Sym}\left(\mathfrak{t}^{*}\right)$ be the product of these weights, where $\operatorname{Sym}\left(\mathfrak{t}^{*}\right)$ denotes the symmetric algebra on $\mathfrak{t}^{*}$. (Here, we say that $f \in \operatorname{Sym}\left(\mathfrak{t}^{*}\right)$ is positive if $(f, \xi)>0$.)

Definition 0.1 Let $(M, \omega, \psi)$ be a Hamiltonian $T$-manifold with discretefixed set, and let $\varphi=\psi^{\xi}$ be a generic component of the moment map. A cohomology class $\alpha_{p} \in H_{T}^{2 \lambda(p)}(M ; A)$ is a canonical class at a fixed point $p$ (with respect to $\varphi$ ) if

(1) $\alpha_{p}(p)=\Lambda_{p}^{-}$.

(2) $\alpha_{p}(q)=0$ for all $q \in M^{T} \backslash\{p\}$ such that $\lambda(q) \leq \lambda(p)$.

Canonical classes do not always exist, but if they exist then they are unique [GT, Lemma 2.7]. Moreover, if there exist canonical classes $\alpha_{p} \in$ $H_{T}^{2 \lambda(p)}(M ; A)$ for all $p \in M^{T}$, then by Lemmas 1.1 and 1.2 below, the classes $\left\{\alpha_{p}\right\}_{p \in M^{T}}$ are a basis for $H_{T}^{*}(M ; A)$ as a module over $H^{*}(B T ; A)$; see also [GT, Proposition 2.3]. In this case, our goal will be to compute the restrictions $\alpha_{p}(q)$ for all $p$ and $q \in M^{T}$ in terms of paths in the canonical graph.

Definition 0.2 Let $(M, \omega, \psi)$ be a Hamiltonian $T$-manifold with discretefixed set, and let $\varphi=\psi^{\xi}$ be a generic component of the moment map. Assume that canonical classes $\alpha_{p} \in H_{T}^{2 \lambda(p)}(M ; A)$ exist for all $p \in M^{T}$. 
There is a labelled directed graph $(V, E)$ associated to $(M, \omega, \psi, \varphi)$, called the canonical graph, defined as follows.

- The vertex set $V$ is the fixed set $M^{T}$; we label each vertex $p \in V$ by its moment image $\psi(p) \in \mathfrak{t}^{*}$.

- The edge set is

$$
E=\left\{\left(r, r^{\prime}\right) \in M^{T} \times M^{T} \mid \lambda\left(r^{\prime}\right)-\lambda(r)=1 \text { and } \alpha_{r}\left(r^{\prime}\right) \neq 0\right\} ;
$$

we label each edge $\left(r, r^{\prime}\right) \in E$ by $\frac{\alpha_{r}\left(r^{\prime}\right)}{\Lambda_{r^{\prime}}^{-}}$.

For example, if $M$ is a Goresky-Kottwitz-MacPherson (GKM) space, then a pair of distinct fixed points $(p, q)$ is an edge in the canonical graph exactly if $\lambda(q)=\lambda(p)+1$ and they are contained in a two-sphere that is fixed by a codimension one subgroup of $T$; see Section 2. Given any directed graph with vertex set $V$ and edge set $E \subset V \times V$, a path of length $k$ from $p$ to $q$ is a $(k+1)$-tuple $\gamma=\left(\gamma_{1}, \ldots, \gamma_{k+1}\right) \in V^{k+1}$, so that $\gamma_{1}=p, \gamma_{k+1}=q$, and $\left(\gamma_{i}, \gamma_{i+1}\right) \in E$ for all $1 \leq i \leq k$. For any path $\gamma$, we let $|\gamma|$ denote its length.

We can now give our most general theorem, which is proved in Section 3. It gives a formula for the restriction of a canonical class $\alpha_{p}$ to a fixed point $q$.

Theorem 0.3. Let $(M, \omega, \psi)$ be a Hamiltonian T-manifold with discretefixed set, and let $\varphi=\psi^{\xi}$ be a generic component of the moment map. Assume that canonical classes $\alpha_{p} \in H_{T}^{2 \lambda(p)}(M ; A)$ exist for all $p \in M^{T}$. Given fixed points $p$ and $q$, let $\Sigma(p, q)$ denote the set of paths from $p$ to $q$ in the associated canonical graph $(V, E)$. Given classes $\mathrm{w}_{r} \in H_{T}^{2}(M ; \mathbb{R})$ for all $r \in M^{T}$,

$$
\alpha_{p}(q)=\Lambda_{q}^{-} \sum_{\gamma \in \Sigma(p, q)} \prod_{i=1}^{|\gamma|} \frac{\mathrm{w}_{\gamma_{i}}\left(\gamma_{i+1}\right)-\mathrm{w}_{\gamma_{i}}\left(\gamma_{i}\right)}{\mathrm{w}_{\gamma_{i}}(q)-\mathrm{w}_{\gamma_{i}}\left(\gamma_{i}\right)} \frac{\alpha_{\gamma_{i}}\left(\gamma_{i+1}\right)}{\Lambda_{\gamma_{i+1}}^{-}},
$$

whenever the right-hand side is well-defined, i.e., $\mathrm{w}_{\gamma_{i}}(q) \neq \mathrm{w}_{\gamma_{i}}\left(\gamma_{i}\right)$ for all $\gamma \in \Sigma(p, q)$ and $1 \leq i \leq|\gamma|$.

This generalizes the formula in $[\mathbf{G T}]$ whenever $H^{2}(M ; \mathbb{R}) \neq \mathbb{R}$; cf. Remark 3.1. This case includes, for example, any generic coadjoint orbit of dimension greater than two. At first glance, the formula above does not look simpler than the one in $[\mathbf{G T}]$ - they both involve sums over the same set of paths. However, a path $\gamma \in \Sigma(p, q)$ contributes 0 to the formula if $\mathbf{w}_{\gamma_{i}}\left(\gamma_{i}\right)=\mathbf{w}_{\gamma_{i}}\left(\gamma_{i+1}\right)$ for some $1 \leq i \leq|\gamma|-1$. Most of our paper (the proof of Theorem 0.3 itself takes less than a page) is dedicated to explaining how to choose the cohomology classes $\mathrm{w}_{r}$ so that only a few paths contribute, and proving that in these cases the formula is manifestly positive whenever the restrictions themselves are all positive (see Remark 3.3).

In Section 4, we show that we can reduce the number of paths whenever there exists a cohomology class w whose restriction to $H^{2}(M ; \mathbb{R})$ is in the 
closure of the Hamiltonian cone (see Definition 4.1) and has the property that $\mathrm{w}(p)=\mathrm{w}(q) \neq \mathrm{w}(r)$ for some edges $(p, q)$ and $(q, r)$ in the canonical graph. For example, if $M$ is a GKM manifold that admits an invariant Kähler structure, then it is enough to find a cohomology class in the closure of the Kähler cone that vanishes on the two-sphere that corresponds to the edge $(p, q)$, but not on the two-sphere that corresponds to $(q, r)$.

In Section 5, we show that our technique is particularly powerful when the manifold is a "strong symplectic fibration" over another Hamiltonian manifold; this class includes, for example, equivariant fiber bundles with the property that the projection map intertwines compatible invariant complex structures. Explicit computations are especially easy in this case. In particular, in Corollary 5.8 we give an inductive formula for the restrictions $\alpha_{p}(q)$ in terms of the paths in the base and the canonical classes on the fiber. Finally, by Theorem 5.5, our formula is integral whenever $M$ is a "tower" of complex projective spaces, that is, a fiber bundle over $\mathbb{C P}^{n}$ whose fiber is also a tower of complex projective spaces. More generally, if the fibers $F_{j}$ are not projective spaces, but do satisfy $H^{*}\left(F_{j} ; A\right) \simeq H^{*}\left(\mathbb{C P}^{n_{j}} ; A\right)$ for some subring $A \subset \mathbb{R}$, then the contributions are all polynomials in the weights with coefficients in $A$.

Since coadjoint orbits of type $A_{n}$ and $C_{n}$ are both towers of complex projective spaces, we immediately get manifestly positive integral formulas for the restrictions in these cases. Similarly, since coadjoint orbits of type $B_{n}$ are towers whose fibers satisfy $H^{*}\left(F_{j} ; \mathbb{Z}\left[\frac{1}{2}\right]\right) \simeq H^{*}\left(\mathbb{C P}^{n_{j}} ; \mathbb{Z}\left[\frac{1}{2}\right]\right)$, the contribution of each path is integral when multiplied by a sufficiently large power of 2. (In a more recent version of $[\mathbf{Z} \mathbf{a}]$, Zara also independently obtained formulas for $C_{n}$ and $B_{n}$ of this type as well.) Finally, coadjoint orbits of type $B_{n}$ and $D_{n}$ are sufficiently close to being towers of complex projective spaces that we can manipulate the terms to get manifestly positive integral formulas in these cases as well.

\section{Canonical classes}

The main goal of this section is to review the properties of canonical classes. However, we also need to prove a slight variation of these results: Lemma 1.4.

Let's begin by recalling a definition. Let $A \subset \mathbb{R}$ be a subring (with unit). The equivariant cohomology of $M$ with coefficients in $A$ is

$$
H_{T}^{*}(M ; A)=H^{*}\left(M \times_{T} E T ; A\right) ;
$$

it is a module over $H^{*}(B T ; A)$. Moreover, the inclusion $M \rightarrow M \times_{T} E T$ induces a natural restriction map $H_{T}^{*}(M ; A) \rightarrow H^{*}(M ; A)$. Here, ET is a contractible space on which $T$ acts freely, and $B T=E T / T$. 
Let $(M, \omega, \psi)$ be a Hamiltonian $T$-manifold with a discrete-fixed set. Given a generic $\xi \in \mathfrak{t}$, the function $\varphi=\psi^{\xi}: M \rightarrow \mathbb{R}$ is an invariant Morse function; the critical set of $\varphi$ is exactly the fixed set $M^{T}$. Our convention for the moment map implies that, for each $p \in M^{T}$, the weights in the negative normal bundle $\nu^{-}(p)$ of $\varphi$ at $p$ are exactly the positive weights of the isotropy action on $T_{p} M$, that is, the weights $\eta$ such that $(\eta, \xi)>0$. Hence, the index of $\varphi$ at $p$ is $2 \lambda(p)$, where $\lambda(p)$ is the number of such weights. In particular, $H^{1}(M ; \mathbb{R})=0$. Finally, given $\alpha \in H_{T}^{*}(M ; A)$ and $q \in M^{T}$, let $\alpha(q)$ denote the image of $\alpha$ under the natural restriction map $H_{T}^{*}(M ; A) \rightarrow H_{T}^{*}(\{q\} ; A)$.

Throughout this paper, we will frequently need the following lemma, which is identical to $[\mathbf{G T}$, Lemma 2.8] except that here we consider coefficients in any subring $A \subset \mathbb{R}$ instead of just $\mathbb{Z}$. The proof goes through without any change.

Lemma 1.1. Let $(M, \omega, \psi)$ be a Hamiltonian T-manifold with discrete-fixed set, and let $\varphi=\psi^{\xi}$ be a generic component of the moment map. Given a canonical class $\alpha_{p} \in H_{T}^{2 \lambda(p)}(M ; A)$ at $p \in M^{T}$,

$$
\alpha_{p}(q)=0 \quad \text { for all } q \in M^{T} \backslash\{p\} \text { such that } \varphi(q) \leq \varphi(p) .
$$

Lemma 1.1 implies that $\varphi(r)<\varphi\left(r^{\prime}\right)$ for all $\left(r, r^{\prime}\right) \in E$. Hence, if $\gamma=$ $\left(\gamma_{1}, \ldots, \gamma_{|\gamma|+1}\right)$ is a path from $p$ to $q$ in $(V, E)$, then $\varphi\left(\gamma_{i}\right)<\varphi(q)$ for all $1 \leq i \leq|\gamma|$.

The following result is due to Kirwan $[\mathbf{K i}]$; see also $[\mathbf{G T}, \mathbf{T W}]$.

Lemma 1.2 (Kirwan). Let $(M, \omega, \psi)$ be a Hamiltonian T-manifold with discrete-fixed set, and let $\varphi=\psi^{\xi}$ be a generic component of the moment map. For every fixed point $p$ there exists a class $\gamma_{p} \in H_{T}^{2 \lambda(p)}(M ; \mathbb{Z})$ so that

(1) $\gamma_{p}(p)=\Lambda_{p}^{-}$, and

(2) $\gamma_{p}(q)=0$ for every $q \in M^{T} \backslash\{p\}$ such that $\varphi(q) \leq \varphi(p)$.

Moreover, for any such classes, the $\left\{\gamma_{p}\right\}_{p \in M^{T}}$ are a basis for $H_{T}^{*}(M ; \mathbb{Z})$ as a module over $H^{*}(B T ; \mathbb{Z})$.

This has the following corollary, which we have adapted from $[\mathbf{G T}$, Corollary 2.6] and [T, Corollary 2.3].

Corollary 1.3. Let $(M, \omega, \psi)$ be a Hamiltonian T-manifold with discretefixed set, and let $\varphi=\psi^{\xi}$ be a generic component of the moment map. Fix $p \in M^{T}$ and $\beta \in H_{T}^{2 i}(M ; A)$ such that $\beta(q)=0$ for all $q \in M^{T}$ satisfying $\varphi(q)<\varphi(p)$.

- $\beta(p)=x \Lambda_{p}^{-}$for some $x \in H^{2 i-2 \lambda(p)}(B T ; A)$; in particular, if $\lambda(p)>i$ then $\beta(p)=0$. 
- Fix cohomology classes $\left\{\gamma_{q}\right\}_{q \in M^{T}}$ so that $\gamma_{q}$ satisfies conditions (1) and (2') above for each $q \in M^{T}$. Then

$$
\beta=\sum_{\varphi(q) \geq \varphi(p)} x_{q} \gamma_{q}, \quad \text { where } x_{q} \in H^{2 i-2 \lambda(q)}(B T ; A) \text { for all } q \text {. }
$$

Here, the sum is over all $q \in M^{T}$ such that $\varphi(q) \geq \varphi(p)$.

We also need the following closely related fact.

Lemma 1.4. Let $(M, \omega, \psi)$ be a Hamiltonian $T$-manifold with discrete-fixed set, and let $\varphi=\psi^{\xi}$ be a generic component of the moment map. Assume that canonical classes $\alpha_{p} \in H_{T}^{2 \lambda(p)}(M ; A)$ exist for all $p \in M^{T}$. Fix $p \in M^{T}$ and $\beta \in H_{T}^{2 i}(M ; A)$ such that $\beta(q)=0$ for all $q \in M^{T}$ so that $\lambda(q)<\lambda(p)$. Then

$$
\beta=\sum_{\lambda(q) \geq \lambda(p)} x_{q} \alpha_{q}, \quad \text { where } x_{q} \in H^{2 i-2 \lambda(q)}(B T ; A) \text { for all } q .
$$

Here the sum is over all $q \in M^{T}$ such that $\lambda(q) \geq \lambda(p)$.

Proof. Since $\left\{\alpha_{q}\right\}_{q \in M^{T}}$ is a basis for $H_{T}^{*}(M ; A)$ as a module over $H^{*}(B T ; A)$, we can write $\beta=\sum_{q \in M^{T}} x_{q} \alpha_{q}$, where $x_{q} \in H^{2 i-2 \lambda(q)}(B T ; A)$ for all $q$. If the claim does not hold, then there exists $q \in M^{T}$ so that $\lambda(q)<\lambda(p)$ and $x_{q} \neq 0$, but $x_{r}=0$ for all $r$ such that $\lambda(r)<\lambda(q)$. Hence, by the definition of canonical class $\beta(q)=x_{q} \Lambda_{q}^{-}$. Since $\beta(q)=0$ this is impossible.

\section{GKM spaces}

We now restrict our attention to an important special case where it is especially easy to calculate canonical classes. A Hamiltonian T-manifold $(M, \omega, \psi)$ is a GKM space if $M$ has isolated fixed points and if, for every codimension one subgroup $K \subset T$, the fixed submanifold $M^{K}$ has dimension at most two $[\mathbf{G K M}]$. Equivalently, $M$ is a GKM space if the weights of the isotropy representation of $T$ on $T_{p} M$ are pairwise linearly independent for every fixed point $p \in M^{T}$.

Definition 2.1 The GKM graph of a $\operatorname{GKM}$ space $(M, \omega, \psi)$ is the labelled directed graph $\left(V, E_{\mathrm{GKM}}\right)$, defined as follows.

- The vertex set $V$ is the fixed set $M^{T}$; we label each $p \in M^{T}$ by its moment image $\psi(p) \in \mathfrak{t}^{*}$.

- Given $p \neq q$ in $V$, there is a directed edge $(p, q) \in E_{\text {GKM }}$ exactly if there exists a codimension one subgroup $K \subset T$ so that $p$ and $q$ are contained in the same connected component $N$ of $M^{K}$. We label each edge $(p, q)$ by the weight $\eta(p, q)$ associated to the isotropy representation of $T$ on $T_{q} N \simeq \mathbb{C}$. 
Observe that $(p, q) \in E_{\mathrm{GKM}}$ exactly if $(q, p) \in E_{\mathrm{GKM}}$. Moreover, $\eta(p, q)=$ $-\eta(q, p)$, and $\psi(q)-\psi(p)$ is a positive multiple of $\eta(p, q)$ for all $(p, q) \in E_{\mathrm{GKM}}$. Additionally, the set of weights of the isotropy representation on the tangent space at any point $p \in V$ is

$$
\Pi_{p}=\Pi_{p}(M)=\left\{\eta(r, p) \mid(r, p) \in E_{\mathrm{GKM}}\right\} .
$$

Example 2.2 The complex projective space $\mathbb{C} P^{n}$. The natural action of $\left(S^{1}\right)^{n+1}$ on $\mathbb{C}^{n+1}$ descends to an effective Hamiltonian action of $T=$ $\left(S^{1}\right)^{n+1} / S^{1}$ on $\mathbb{C} P^{n}$. The associated GKM graph is the complete graph on $n+1$ fixed points: $p_{1}=[1,0, \ldots, 0], p_{2}=[0,1, \ldots, 0], \ldots, p_{n+1}=$ $[0,0, \ldots, 1]$. Finally, the moment image of $p_{i}$ is $\frac{1}{n+1} \sum_{j=1}^{n+1}\left(x_{j}-x_{i}\right)$, and the weight associated to the edge $\left(p_{i}, p_{j}\right)$ is $x_{i}-x_{j}$. Here, we let $x_{1}, \ldots, x_{n+1}$ be the standard basis for $\left(\mathbb{R}^{n+1}\right)^{*}$ and identify $\mathfrak{t}^{*}$ with $\left\{\mu \in\left(\mathbb{R}^{n+1}\right)^{*} \mid \sum \mu_{i}=0\right\}$.

Now fix a generic component of the moment map $\varphi=\psi^{\xi}$. As we mentioned in the previous section, the set of weights in the isotropy representation on the negative normal bundle of $\varphi$ at $p$ is the set of positive weights in $\Pi_{p}(M)$. Hence, $\lambda(p)$ is the number of edges $(r, p) \in E_{\mathrm{GKM}}$ such that $\varphi(r)<\varphi(p)$, and

$$
\Lambda_{p}^{-}=\prod_{\substack{\eta \in \Pi_{p}(M) \\(\eta, \xi)>0}} \eta .
$$

It is possible to strengthen Lemma 1.1 when $M$ is a GKM space. We say that a path $\gamma=\left(\gamma_{1}, \ldots, \gamma_{|\gamma|+1}\right)$ in $\left(V, E_{\mathrm{GKM}}\right)$ is ascending if $\varphi\left(\gamma_{i}\right)<\varphi\left(\gamma_{i+1}\right)$ for all $i$.

Lemma 2.3. Let $(M, \omega, \psi)$ be a GKM space, and let $\varphi=\psi^{\xi}$ be a generic component of the moment map. Given a canonical class $\alpha_{p} \in H_{T}^{2 \lambda(p)}(M ; A)$ at $p \in M^{T}, \alpha_{p}(q)=0$ for all $q \in M^{T}$ such that there are no ascending paths from $p$ to $q$ in $\left(V, E_{\mathrm{GKM}}\right)$.

Proof. Consider any $q \in M^{T}$ so that $\alpha_{p}(q) \neq 0$ but $\alpha_{p}(r)=0$ for each edge $(r, q) \in E_{\mathrm{GKM}}$ such that $\varphi(r)<\varphi(q)$. Then $\alpha_{p}(q)$ is a non-zero multiple of $\eta(r, q)$ for all $(r, q) \in E_{\mathrm{GKM}}$ such that $\varphi(r)<\varphi(q)$. (To see this, recall that for each $(r, q) \in E_{\mathrm{GKM}}$ there exists a sphere $N \subset M$ containing $r$ and $q$ which is fixed by the codimension one subgroup associated to $\eta$.) Since these weights are pairwise linearly independent, this implies that $\alpha_{p}$ has degree at least $2 \lambda(q)$, that is, $\lambda(q) \leq \lambda(p)$. By the definition of canonical class, this is impossible unless $p=q$. The claim follows.

We say that $\varphi$ is index increasing if $\lambda(p)<\lambda(q)$ for every edge $(p, q) \in$ $E_{\mathrm{GKM}}$ such that $\varphi(p)<\varphi(q)$. In this case, integral canonical classes exist and it is straightforward to compute the restriction of a canonical class $\alpha_{p}$ 
to $q$ for any $p$ and $q$ in $M^{T}$ such that $\lambda(q)-\lambda(p)=1$. Conversely, if there exist canonical classes $\alpha_{p} \in H_{T}^{*}(M ; \mathbb{Q})$ for all $p \in M^{T}$, then $\varphi$ is index increasing [GT, Remark 4.2].

More specifically, let $\xi^{\circ}=\left\{\beta \in \mathfrak{t}^{*} \mid(\beta, \xi)=0\right\}$. Given $\eta \in \mathfrak{t}^{*}$, let $\varrho_{\eta}: \operatorname{Sym}\left(\mathfrak{t}^{*}\right) \rightarrow \operatorname{Sym}\left(\mathfrak{t}^{*}\right)$ be the homomorphism of symmetric algebras induced by the projection map which sends $X \in \mathfrak{t}^{*}$ to $X-\frac{(X, \xi)}{(\eta, \xi)} \eta \in \xi^{\circ} \subset \mathfrak{t}^{*}$. Following $[\mathbf{G Z}]$, for any $(p, q) \in E_{\mathrm{GKM}}$ we define

$$
\Theta(p, q)=\frac{\varrho_{\eta(p, q)}\left(\Lambda_{p}^{-}\right)}{\varrho_{\eta(p, q)}\left(\frac{\Lambda_{q}^{-}}{\eta(p, q)}\right)} \in \operatorname{Sym}\left(\mathfrak{t}^{*}\right)_{0},
$$

where $\operatorname{Sym}\left(\mathfrak{t}^{*}\right)_{0}$ denotes the ring of fractions of $\operatorname{Sym}\left(\mathfrak{t}^{*}\right)$. Observe that $\varrho_{\eta(p, q)}\left(\frac{\Lambda_{q}^{-}}{\eta(p, q)}\right)$ is not zero, since by the GKM assumption the weights at each fixed point are pairwise linearly independent. The theorem below was proved in $[\mathbf{G Z}]$ over the rationals and then extended to the integers in $[\mathbf{G T}]$.

Theorem 2.4. Let $(M, \omega, \psi)$ be a GKM space, and let $\left(V, E_{\mathrm{GKM}}\right)$ be the associated GKM graph. Let $\varphi=\psi^{\xi}$ be a generic component of the moment map; assume that $\varphi$ is index increasing. Then

(1) There exist canonical classes $\alpha_{p} \in H_{T}^{2 \lambda(p)}(M ; \mathbb{Z})$ for all $p \in M^{T}$.

(2) Given fixed points $p$ and $q$ such that $\lambda(q)-\lambda(p)=1$,

$$
\alpha_{p}(q)= \begin{cases}\Lambda_{q}^{-} \frac{\Theta(p, q)}{\eta(p, q)} & \text { if }(p, q) \in E_{\mathrm{GKM}}, \text { and } \\ 0 & \text { if }(p, q) \notin E_{\mathrm{GKM}} .\end{cases}
$$

(3) $\Theta(p, q) \in \mathbb{Z} \backslash\{0\}$ for all $(p, q) \in E_{\mathrm{GKM}}$ such that $\lambda(q)-\lambda(p)=1$.

In particular, the associated canonical graph has vertex set $V=M^{T}$ and edge set

$$
E=\left\{\left(r, r^{\prime}\right) \in E_{\mathrm{GKM}} \mid \lambda\left(r^{\prime}\right)-\lambda(r)=1\right\} .
$$

\section{The most general theorem}

In this section, we will prove our most general theorem, Theorem 0.3. As we mentioned in the introduction, it is a generalization of [GT, Theorem 1.2]. More precisely, it is more general whenever $H^{2}(M ; \mathbb{R}) \neq \mathbb{R}$; see Remark 3.1. The main advantage of our formula is that it usually allows us to express $\alpha_{p}(q)$ as a sum over fewer paths. For the reader's convenience, we will recall the statement of Theorem 0.3.

Let $(M, \omega, \psi)$ be a Hamiltonian T-manifold with discrete-fixed set, and let $\varphi=\psi^{\xi}$ be a generic component of the moment map. 
Assume that canonical classes $\alpha_{p} \in H_{T}^{2 \lambda(p)}(M ; A)$ exist for all $p \in M^{T}$. Given fixed points $p$ and $q$, let $\Sigma(p, q)$ denote the set of paths from $p$ to $q$ in the associated canonical graph $(V, E)$. Given classes $\mathrm{w}_{r} \in H_{T}^{2}(M ; \mathbb{R})$ for all $r \in M^{T}$,

$$
\alpha_{p}(q)=\Lambda_{q}^{-} \sum_{\gamma \in \Sigma(p, q)} \prod_{i=1}^{|\gamma|} \frac{\mathrm{w}_{\gamma_{i}}\left(\gamma_{i+1}\right)-\mathrm{w}_{\gamma_{i}}\left(\gamma_{i}\right)}{\mathrm{w}_{\gamma_{i}}(q)-\mathrm{w}_{\gamma_{i}}\left(\gamma_{i}\right)} \frac{\alpha_{\gamma_{i}}\left(\gamma_{i+1}\right)}{\Lambda_{\gamma_{i+1}}^{-}},
$$

whenever the right-hand side is well-defined, i.e., $\mathrm{w}_{\gamma_{i}}(q) \neq$ $\mathrm{w}_{\gamma_{i}}\left(\gamma_{i}\right)$ for all $\gamma \in \Sigma(p, q)$ and $1 \leq i \leq|\gamma|$.

Remark 3.1. By Lemma 1.1, $\varphi\left(\gamma_{i}\right)<\varphi(q)$ for all $\gamma \in \Sigma(p, q)$ and $1 \leq i \leq$ $|\gamma|$; a fortiori, $\psi\left(\gamma_{i}\right) \neq \psi(q)$. Therefore, the right-hand side of the equation above is well-defined if $\mathrm{w}_{r}$ is a non-zero multiple of $[\omega+\psi]$ for all $r \in M^{T}$. (Here we are using the Cartan model for the equivariant cohomology of $M$.) In this case, the theorem agrees with [GT, Theorem 1.2].

Note that a path $\gamma \in \Sigma(p, q)$ contributes 0 to the formula above exactly if there exists $1 \leq i \leq|\gamma|-1$ such that $\mathrm{w}_{\gamma_{i}}\left(\gamma_{i}\right)=\mathrm{w}_{\gamma_{i}}\left(\gamma_{i+1}\right)$ but $\mathrm{w}_{\gamma_{i}}(q) \neq$ $\mathrm{w}_{\gamma_{i}}\left(\gamma_{i}\right)$. Generally speaking, the best result will come from choosing each class $\mathrm{w}_{r}$ so that $\mathrm{w}_{r}(r) \neq \mathrm{w}_{r}(q)$, but $\mathrm{w}_{r}(r)=\mathrm{w}_{r}(s)$ for as many edges $(r, s) \in$ $E$ as possible. In practice, instead of trying to pick the optimal class at each fixed point, we will often fix an ordered list of classes. For each fixed point we will just pick the first class that satisfies the hypotheses of Theorem 0.3. As we show below, as long as the forms satisfy the technical condition (1), this technique gives an elegant answer. In the next two sections, we will explain natural geometric conditions which guarantee that (1) is satisfied.

Corollary 3.2. Let $(M, \omega, \psi)$ be a Hamiltonian T-manifold with discretefixed set, and let $\varphi=\psi^{\xi}$ be a generic component of the moment map. Assume that canonical classes $\alpha_{p} \in H_{T}^{2 \lambda(p)}(M ; A)$ exist for all $p \in M^{T}$. Pick classes ${ }^{1}$ $\mathrm{w}_{1}, \mathrm{w}_{2}, \ldots, \mathrm{w}_{k}$ in $H_{T}^{2}(M ; \mathbb{R})$ such that, for each $j$,

(1) $\alpha_{p}(q)=0 \forall p, q \in M^{T}$ such that $\mathrm{w}_{j}(q) \neq \mathrm{w}_{j}(p)$ and $\mathrm{w}_{j}^{\xi}(q) \leq \mathrm{w}_{j}^{\xi}(p)$,

where for each $p \in M^{T}, \mathrm{w}_{j}^{\xi}(p)$ denotes $\left(\mathrm{w}_{j}(p), \xi\right)$.

Assume that for each $\left(r, r^{\prime}\right) \in E$, there exists $j \in\{1, \ldots, k\}$ such that $\mathrm{w}_{j}(r) \neq \mathrm{w}_{j}\left(r^{\prime}\right)$, and define

$$
h\left(r, r^{\prime}\right)=\min \left\{j \mid \mathrm{w}_{j}(r) \neq \mathrm{w}_{j}\left(r^{\prime}\right)\right\} \quad \text { for all }\left(r, r^{\prime}\right) \in E .
$$

\footnotetext{
${ }^{1}$ In practice, there often exists a symplectic form $\omega_{j} \in \Omega^{2}(M)$ with moment map $\psi_{j}: M \rightarrow \mathfrak{t}^{*}$ such that $\left[\omega_{j}+\psi_{j}\right]=\mathrm{w}_{j} \in H_{T}^{2}(M ; \mathbb{R})$ for each $j$. In this case, $\mathrm{w}_{j}(p)=\psi_{j}(p)$ for all $p \in M^{T}$; in particular, $\mathbf{w}_{j}^{\xi}(p)=\psi_{j}^{\xi}(p)$. However, we do not insist that such symplectic forms exist; we allow the general case.
} 
Given $p$ and $q$ in $M^{T}$, let $\Sigma(p, q)$ denote the set of paths in the associated canonical graph $(V, E)$ from $p$ to $q$. Then

$$
\begin{aligned}
\alpha_{p}(q) & =\Lambda_{q}^{-} \sum_{\gamma \in C(p, q)} \prod_{i=1}^{|\gamma|} \frac{\mathrm{w}_{h\left(\gamma_{i}, \gamma_{i+1}\right)}\left(\gamma_{i+1}\right)-\mathrm{w}_{h\left(\gamma_{i}, \gamma_{i+1}\right)}\left(\gamma_{i}\right)}{\mathrm{w}_{h\left(\gamma_{i}, \gamma_{i+1}\right)}(q)-\mathrm{w}_{h\left(\gamma_{i}, \gamma_{i+1}\right)}\left(\gamma_{i}\right)} \frac{\alpha_{\gamma_{i}}\left(\gamma_{i+1}\right)}{\Lambda_{\gamma_{i+1}}^{-}}, \text {where } \\
C(p, q) & =\left\{\gamma \in \Sigma(p, q) \mid h\left(\gamma_{1}, \gamma_{2}\right) \leq h\left(\gamma_{2}, \gamma_{3}\right) \leq \cdots \leq h\left(\gamma_{|\gamma|}, \gamma_{|\gamma|+1}\right)\right\} .
\end{aligned}
$$

Remark 3.3 (Positivity). Note that, if $\alpha_{r}\left(r^{\prime}\right)$ is positive for every edge $\left(r, r^{\prime}\right)$ in $E$, then the equation above is manifestly positive, in the sense that every non-zero term is positive; a fortiori, the restriction $\alpha_{p}(q)$ is positive for all fixed points $p$ and $q$. To see this, note that $\Lambda_{r}^{-}$is positive by definition for all $r \in M^{T}$, while $\mathrm{w}_{i}\left(r^{\prime}\right)-\mathrm{w}_{i}(r)$ is either positive or zero for each edge $\left(r, r^{\prime}\right) \in E$ by assumption (1). Therefore, the formulas in Theorem 4.2 and Theorem 5.5. that are both proved using the above corollary, a manifestly positive whenever the restrictions themselves are manifestly positive. In contrast, in general the restriction $\alpha_{p}(q)$ might not be positive (cf. [GT, Example 5.2]).

We are now ready to prove our claims.

Proof of Theorem 0.3 . Since $\left(\mathrm{w}_{p}-\mathrm{w}_{p}(p)\right)(p)=0$ and $\alpha_{p}$ is a canonical class at $p$, the restriction $\alpha_{p}\left(\mathrm{w}_{p}-\mathrm{w}_{p}(p)\right)(r)$ is trivial for all $r \in M^{T}$ such that $\lambda(r) \leq \lambda(p)$. By Lemma 1.4, this implies that we can write

$$
\alpha_{p}\left(\mathrm{w}_{p}-\mathrm{w}_{p}(p)\right)=\sum_{\lambda(r)>\lambda(p)} x_{r} \alpha_{r}, \text { where } x_{r} \in H^{2 \lambda(p)-2 \lambda(r)+2}(B T ; \mathbb{R}) \forall r .
$$

By the definition of canonical class, evaluating the above equation at $r$ implies that

$$
\left(\mathrm{w}_{p}(r)-\mathrm{w}_{p}(p)\right) \frac{\alpha_{p}(r)}{\Lambda_{r}^{-}}=x_{r} \in \mathbb{R} \text { for all } r \in M^{T} \text { such that } \lambda(r)=\lambda(p)+1
$$

Moreover, by dimensional arguments, $x_{r}=0$ for all $r \in M^{T}$ such that $\lambda(r)>\lambda(p)+1$. Hence,

$$
\alpha_{p}\left(\mathrm{w}_{p}-\mathrm{w}_{p}(p)\right)=\sum_{(p, r) \in E}\left(\mathrm{w}_{p}(r)-\mathrm{w}_{p}(p)\right) \frac{\alpha_{p}(r)}{\Lambda_{r}^{-}} \alpha_{r}
$$

Restricting to $q$ and dividing by $\mathrm{w}_{p}(q)-\mathrm{w}_{p}(p)$ (which is not zero by assumption), we have

$$
\alpha_{p}(q)=\sum_{(p, r) \in E} \frac{\mathrm{w}_{p}(r)-\mathrm{w}_{p}(p)}{\mathrm{w}_{p}(q)-\mathrm{w}_{p}(p)} \frac{\alpha_{p}(r)}{\Lambda_{r}^{-}} \alpha_{r}(q) .
$$

Since the claim is obvious if $\lambda(q)-\lambda(p) \leq 1$, the claim now follows by induction. 
Proof of Corollary 3.2. Definition 0.2 and hypothesis (1) in Corollary 3.2 imply that

either $\mathrm{w}_{j}^{\xi}(r)<\mathrm{w}_{j}^{\xi}\left(r^{\prime}\right)$ or $\mathrm{w}_{j}(r)=\mathrm{w}_{j}\left(r^{\prime}\right)$ for all $\left(r, r^{\prime}\right) \in E$ and $1 \leq j \leq k$.

Therefore, if $\gamma$ is a path from $r$ to $q$ with at least one edge then either $\mathrm{w}_{j}^{\xi}\left(\gamma_{i}\right)<\mathrm{w}_{j}^{\xi}(q)$ or $\mathrm{w}_{j}\left(\gamma_{i}\right)=\mathrm{w}_{j}\left(\gamma_{i+1}\right)=\mathrm{w}_{j}(q)$ for each $j$ and for each $i \leq|\gamma|$. Since, by assumption, there exists $j \in\{1, \ldots, k\}$ such that $\mathrm{w}_{j}\left(\gamma_{1}\right) \neq \mathrm{w}_{j}\left(\gamma_{2}\right)$, this implies that $\mathrm{w}_{j}^{\xi}(r)<\mathrm{w}_{j}^{\xi}(q)$. A fortiori, $\mathrm{w}_{j}(r) \neq \mathrm{w}_{j}(q)$, and so we can define

$$
h(r, q)=\min \left\{j \mid \mathrm{w}_{j}(r) \neq \mathrm{w}_{j}(q)\right\} \forall r \in M^{T} \backslash\{q\} \text { such that } \Sigma(r, q) \neq \emptyset .
$$

Moreover, if $\mathbf{w}_{j}\left(\gamma_{i}\right)=\mathbf{w}_{j}(q)$ for some $\gamma \in \Sigma(p, q)$ and $i \leq|\gamma|$, then $\mathbf{w}_{j}\left(\gamma_{i}\right)=$ $\mathrm{w}_{j}\left(\gamma_{i+1}\right)=\mathrm{w}_{j}(q)$ as well. Therefore,

(2) $h\left(\gamma_{i}, q\right) \leq h\left(\gamma_{i+1}, q\right)$ and $h\left(\gamma_{i}, q\right) \leq h\left(\gamma_{i}, \gamma_{i+1}\right)$ for all $1 \leq i \leq|\gamma|-1$.

The hypotheses of Theorem 0.3 will be satisfied if we let the class associated to $r \in M^{T}$ be

$$
\begin{cases}\mathrm{w}_{h(r, q)} & \text { if } r \neq q \text { and } \Sigma(r, q) \neq \emptyset, \text { and } \\ 0 & \text { otherwise. }\end{cases}
$$

Therefore,

$$
\alpha_{p}(q)=\Lambda_{q}^{-} \sum_{\gamma \in \Sigma(p, q)} \prod_{i=1}^{|\gamma|} \frac{\mathrm{w}_{h\left(\gamma_{i}, q\right)}\left(\gamma_{i+1}\right)-\mathrm{w}_{h\left(\gamma_{i}, q\right)}\left(\gamma_{i}\right)}{\mathrm{w}_{h\left(\gamma_{i}, q\right)}(q)-\mathrm{w}_{h\left(\gamma_{i}, q\right)}\left(\gamma_{i}\right)} \frac{\alpha_{\gamma_{i}}\left(\gamma_{i+1}\right)}{\Lambda_{\gamma_{i+1}}^{-}} .
$$

Moreover, a path $\gamma \in \Sigma(p, q)$ contributes 0 to the formula above if $\mathrm{w}_{h\left(\gamma_{i}, q\right)}\left(\gamma_{i}\right)=\mathrm{w}_{h\left(\gamma_{i}, q\right)}\left(\gamma_{i+1}\right)$ for some $i<|\gamma|$. Therefore, we only need to consider paths $\gamma$ from $p$ to $q$ so that

$$
h\left(\gamma_{i}, \gamma_{i+1}\right) \leq h\left(\gamma_{i}, q\right) \quad \text { for all } 1 \leq i \leq|\gamma| .
$$

Combining this fact together with (2), we may restrict to paths $\gamma$ so that

$$
\begin{gathered}
h\left(\gamma_{1}, \gamma_{2}\right) \leq h\left(\gamma_{2}, \gamma_{3}\right) \leq \cdots \leq h\left(\gamma_{|\gamma|}, \gamma_{|\gamma|+1}\right) \quad \text { and } \\
h\left(\gamma_{i}, \gamma_{i+1}\right)=h\left(\gamma_{i}, q\right), \quad \text { for all } 1 \leq i \leq|\gamma| .
\end{gathered}
$$

Finally, the lemma below, which we will use in Section 5, follows from an argument nearly identical to the first three sentences of the proof of Theorem 0.3.

Lemma 3.4. Let $(M, \omega, \psi)$ be a Hamiltonian T-manifold with discrete-fixed set, and let $\varphi=\psi^{\xi}$ be a generic component of the moment map. Assume 
that canonical classes $\alpha_{p} \in H_{T}^{2 \lambda(p)}(M ; A)$ exist for all $p \in M^{T}$; let $(V, E)$ be the canonical graph. Given a class $\mathrm{w} \in H_{T}^{2}(M ; A)$,

$$
(\mathrm{w}(r)-\mathrm{w}(p)) \frac{\alpha_{p}(r)}{\Lambda_{r}^{-}} \in A \quad \text { for all }(p, r) \in E
$$

\section{The Hamiltonian cone}

In this section, we give our first application of Theorem 0.3. Here, we use the Hamiltonian cone to pick the closed equivariant two-forms and characterize which paths contribute to the formula.

Definition 4.1 Let a torus $T$ act on a manifold $M$. The Hamiltonian cone is the set of classes $\mathcal{H} \subset H^{2}(M ; \mathbb{R})$, which can be represented by an invariant symplectic form that has a moment map.

Theorem 4.2. Let $(M, \omega, \psi)$ be a Hamiltonian T-manifold with discretefixed set, and let $\varphi=\psi^{\xi}$ be a generic component of the moment map. Assume that canonical classes $\alpha_{p} \in H_{T}^{2 \lambda(p)}(M ; A)$ exist for all $p \in M^{T}$. Pick classes $\mathrm{w}_{1}, \ldots, \mathrm{w}_{k} \in H_{T}^{2}(M ; \mathbb{R})$ that restrict to classes in the closure of the component of the Hamiltonian cone $\mathcal{H} \subset H^{2}(M ; \mathbb{R})$ containing $[\omega]$. Assume that for each $\left(r, r^{\prime}\right) \in E$ there exists $j$ such that $\mathrm{w}_{j}(r) \neq \mathrm{w}_{j}\left(r^{\prime}\right)$, and define

$$
h\left(r, r^{\prime}\right)=\min \left\{j \mid \mathrm{w}_{j}(r) \neq \mathrm{w}_{j}\left(r^{\prime}\right)\right\} \quad \text { for all }\left(r, r^{\prime}\right) \in E .
$$

Given $p$ and $q$ in $M^{T}$, let $\Sigma(p, q)$ denote the set of paths from $p$ to $q$ in the associated canonical graph $(V, E)$. Then

$$
\begin{aligned}
\alpha_{p}(q) & =\Lambda_{q}^{-} \sum_{\gamma \in C(p, q)} \prod_{i=1}^{|\gamma|} \frac{\mathrm{w}_{h\left(\gamma_{i}, \gamma_{i+1}\right)}\left(\gamma_{i+1}\right)-\mathrm{w}_{h\left(\gamma_{i}, \gamma_{i+1}\right)}\left(\gamma_{i}\right)}{\mathrm{w}_{h\left(\gamma_{i}, \gamma_{i+1}\right)}(q)-\mathrm{w}_{h\left(\gamma_{i}, \gamma_{i+1}\right)}\left(\gamma_{i}\right)} \frac{\alpha_{\gamma_{i}}\left(\gamma_{i+1}\right)}{\Lambda_{\gamma_{i+1}}^{-}}, \text {where } \\
C(p, q) & =\left\{\gamma \in \Sigma(p, q) \mid h\left(\gamma_{1}, \gamma_{2}\right) \leq h\left(\gamma_{2}, \gamma_{3}\right) \leq \cdots \leq h\left(\gamma_{|\gamma|}, \gamma_{|\gamma|+1}\right)\right\} .
\end{aligned}
$$

Remark 4.3. Assume that the following conditions hold:

(X) the restrictions of the $\mathrm{w}_{i}$ form a basis for $H^{2}(M ; \mathbb{R})$; and

(Y) the restriction of $\sum_{i} a_{i} \mathrm{w}_{i}$ lies in $\mathcal{H}$ for every positive $k$-tuple $a \in \mathbb{R}_{+}^{k}$.

In this case, Theorem 4.2 can also be proved using the limit techniques found in $[\mathbf{Z a}$ ], instead of Theorem 0.3. (The argument still relies on Lemmas 4.5 and 4.6.) Note that, in this case, we do not need to assume that for each $\left(r, r^{\prime}\right) \in E$ there exists $j$ such that $\mathrm{w}_{j}(r) \neq \mathrm{w}_{j}\left(r^{\prime}\right)$; this holds automatically.

Remark 4.4. Assume that the torus $T$ acts on a compact manifold $M$, preserving a complex structure $J: T M \rightarrow T M$. If the fixed set is empty the Hamiltonian cone $\mathcal{H}$ is empty, so assume that $M^{T} \neq \emptyset$. The Kähler cone of 
$M$ is the set of classes in $H^{2}(M ; \mathbb{R})$ which can be represented by a compatible symplectic form. Since $T$ is compact, we can represent every such class by an invariant symplectic form by averaging. Moreover, by Frankel's theorem, the action is always Hamiltonian. Hence, the Kähler cone is a subset of the Hamiltonian cone. (Note that Lemma 4.5 is obvious if $\left[\omega^{\prime}\right]$ is in the Kähler cone containing $[\omega]$.) Analogous statements hold if $J$ is an almost complex structure and $H^{1}(M ; \mathbb{R})=0$.

Note also that the Kähler cone is convex because any convex combination of compatible symplectic forms is itself a compatible symplectic form. In contrast, a convex combination of arbitrary symplectic forms may or may not be symplectic.

Lemma 4.5. Let $(M, \omega, \psi)$ be a Hamiltonian $T$-manifold, and let $\varphi=\psi^{\xi}$ be a generic component of the moment map. Let $\omega^{\prime}$ be a symplectic form on $M$ with moment $\psi^{\prime}$ so that $\left[\omega^{\prime}\right]$ lies in the component of $\mathcal{H} \subset H^{2}(M ; \mathbb{R})$ containing $[\omega]$. Then $\left(\Lambda_{p}^{\prime}\right)^{-}$, the product of the positive weights of the isotropy representation of $T$ on $\left(T_{p} M, \omega^{\prime}\right)$, is $\Lambda_{p}^{-}$for all $p \in M^{T}$.

Proof. Let $\varphi^{\prime}=\left(\psi^{\prime}\right)^{\xi}$. It is sufficient to prove the claim for all $\omega^{\prime}$ such that $\left[\omega^{\prime}\right]$ lies in some neighborhood of $[\omega]$. Therefore, we may assume that

$$
\varphi(r)<\varphi(s) \Rightarrow \varphi^{\prime}(r)<\varphi^{\prime}(s), \quad \text { for all } r \text { and } s \in M^{T} .
$$

Fix $p \in M^{T}$. By applying Lemma 1.2 to $\varphi$, there exists a class $\gamma_{p} \in$ $H_{T}^{2 \lambda(p)}(M ; \mathbb{Z})$ so that $\gamma_{p}(p)=\Lambda_{p}^{-}$and $\gamma_{p}(q)=0$ for every $q \in M^{T} \backslash\{p\}$ such that $\varphi(q) \leq \varphi(p)$. By the assumption above, this implies that $\gamma_{p}(q)=0$ for every $q \in M^{T} \backslash\{p\}$ such that $\varphi^{\prime}(q)<\varphi^{\prime}(p)$. By applying Corollary 1.3 to $\varphi^{\prime}$, this implies that $\Lambda_{p}^{-}=\gamma_{p}(p)$ is a multiple of $\left(\Lambda_{p}^{\prime}\right)^{-}$. Since a nearly identical argument shows that $\left(\Lambda_{p}^{\prime}\right)^{-}$is a multiple of $\Lambda_{p}^{-}$, the claim follows from the fact that these are both positive.

Lemma 4.6. Let $(M, \omega, \psi)$ be a Hamiltonian $T$-manifold with discrete-fixed set, and let $\varphi=\psi^{\xi}$ be a generic component of the moment map. Fix a class $\mathrm{w} \in H_{T}^{2}(M ; \mathbb{R})$ that restricts to a class in the closure of the component of $\mathcal{H} \subset H^{2}(M ; \mathbb{R})$ containing $[\omega]$. Given a canonical class $\alpha_{p} \in H_{T}^{2 \lambda(p)}(M ; A)$ at $p \in M^{T}$ (with respect to $\varphi$ ),

$\alpha_{p}(q)=0 \quad$ for all $p$ and $q \in M^{T}$ such that $\mathrm{w}(q) \neq \mathrm{w}(p)$ and $\mathrm{w}^{\xi}(q) \leq \mathrm{w}^{\xi}(p)$.

Proof. By perturbing $\xi$ slightly, if necessary, we may assume that $\mathrm{w}(p)=$ $\mathrm{w}(q)$ exactly if $\mathrm{w}^{\xi}(p)=\mathrm{w}^{\xi}(q)$ for all $p$ and $q$ in $M^{T}$. Hence, there exists $\epsilon>0$ so that $\mathrm{w}^{\xi}(q)<\mathrm{w}^{\xi}(p)-\epsilon$ for all $p$ and $q \in M^{T}$ such that $\mathrm{w}(q) \neq \mathrm{w}(p)$ and $w^{\xi}(q) \leq w^{\xi}(p)$. By assumption, there exists a symplectic form $\omega^{\prime}$ with 
moment map $\psi^{\prime}$ so that

$$
\left|\left(\psi^{\prime}\right)^{\xi}(p)-\mathrm{w}^{\xi}(p)\right|<\frac{1}{2} \epsilon \text { for all } p \in M^{T}
$$

and $\left[\omega^{\prime}\right]$ lies in the closure of the component of $\mathcal{H} \subset H^{2}(M ; \mathbb{R})$ containing $[\omega]$. By Lemma 4.5, the latter fact implies that the product of the positive weights for the isotropy action on $\left(T_{p} M, \omega^{\prime}\right)$ is $\Lambda_{p}^{-}$for all $p \in M^{T}$. Hence, by the definition of canonical class, $\alpha_{p}$ is also the canonical class at $p$ with respect to $\varphi^{\prime}$. By Lemma 1.1, this implies that

$$
\alpha_{p}(q)=0 \text { for all } p \text { and } q \in M^{T} \text { such that }\left(\psi^{\prime}\right)^{\xi}(q)<\left(\psi^{\prime}\right)^{\xi}(p) \text {. }
$$

Finally, (3) implies that $\left(\psi^{\prime}\right)^{\xi}(q)<\left(\psi^{\prime}\right)^{\xi}(p)$ for all $q \in M^{T}$ such that $\mathrm{w}^{\xi}(q)<$ $\mathrm{w}^{\xi}(p)-\epsilon$.

Proof of Theorem 4.2. The claim follows immediately from Lemma 4.6 and Corollary 3.2.

Finally, we make the following observation, which we will not need in this paper.

Lemma 4.7. Let $(M, \omega, \psi)$ be a Hamiltonian T-manifold. Let $\omega^{\prime}$ be a symplectic form on $M$ with moment map $\psi^{\prime}$ so that $\left[\omega^{\prime}\right]$ lies in the component of $\mathcal{H} \subset H^{2}(M ; \mathbb{R})$ containing $[\omega]$. Then $c_{1}(M)=c_{1}^{\prime}(M)$, where $c_{1}(M)$ and $c_{1}^{\prime}(M) \in H_{T}^{2}(M ; \mathbb{Z})$ are the first equivariant Chern class associated to $\omega$ and $\omega^{\prime}$, respectively.

Proof. Let $\varphi=\psi^{\xi}$ be a generic component of the moment map. Since the weights in the representations $\left(T_{q} M, \omega\right)$ and $\left(T_{q} M, \omega^{\prime}\right)$ agree up to sign, Lemma 4.5 implies immediately that $c_{1}(M)(q)=c_{1}^{\prime}(M)(q)$ for all $q \in M^{T}$ such that $\lambda(q) \leq 1$. By Lemma 1.4, this implies that $c_{1}(M)-c_{1}^{\prime}(M)=0$.

\section{Fiber bundles}

In this section, we show how to use Theorem 0.3 (and Corollary 3.2) to get effective formulas for the restrictions $\alpha_{p}(q)$ in the case that our Hamiltonian $T$-manifold is a fiber bundle over a Hamiltonian $T$-manifold (and certain technical restrictions hold). In certain very nice cases, such as when $M$ is a "tower of complex projective spaces" (see Definition 5.4) and the restrictions of the canonical classes are positive, the contribution from each path will be a positive integer multiple of the product of positive weights. More precisely, let $(M, \omega, \psi)$ and $(\widetilde{M}, \widetilde{\omega}, \widetilde{\psi})$ be Hamiltonian $T$-manifolds. We will consider the following maps. 
Definition 5.1 A map $\pi: M \rightarrow \widetilde{M}$ is a strong symplectic fibration ${ }^{2}$ if

(1) the map $\pi$ is an equivariant fiber bundle with symplectic fibers, that is, the restriction of $\omega$ to the fiber $\widehat{M}_{p}=\pi^{-1}(\pi(p))$ is symplectic for all $p \in M ;$ and

(2) as symplectic representations $\left(T_{p} M, \omega\right) \simeq\left(T_{p} \widehat{M}_{p},\left.\omega\right|_{\widehat{M}_{p}}\right) \oplus\left(T_{\pi(p)} \widetilde{M}, \widetilde{\omega}\right)$ for all $p \in M^{T}$.

Example 5.2 There are several situations where an equivariant fiber bundle $\pi: M \rightarrow \widetilde{M}$ is automatically a strong symplectic fibration.

(i) Let $J$ and $\widetilde{J}$ be compatible almost complex structures on $M$ and $\widetilde{M}$, respectively. If $\pi: M \rightarrow \widetilde{M}$ intertwines $J$ and $\widetilde{J}$, i.e. $d \pi \circ J=\widetilde{J} \circ d \pi$, then $\pi$ is a strong symplectic fibration. The fibers are symplectic because $T_{p} \widehat{M}_{p}$ is $J$ invariant for all $p \in M$. For all $p \in M^{T}$, the symplectic perpendicular $H_{p}=\left(T_{p} \widehat{M}_{p}\right)^{\omega}$ is a complex subspace and $T_{p} M=T_{p} \widehat{M}_{p} \oplus H_{p}$ as complex representations. Finally, $\pi$ induces an isomorphism of $\left(H_{p},\left.\omega\right|_{H_{p}}\right)$ and $\left(T_{\pi(p)} \widetilde{M}, \widetilde{\omega}\right)$ as symplectic representations.

(ii) If $\pi$ has symplectic fibers and $\omega_{\mu}=\mu \omega+(1-\mu) \pi^{*}(\widetilde{\omega})$ is symplectic for all $\mu \in(0,1]$, then $\pi$ is a strong symplectic fibration. Since $(0,1]$ is connected, $\left(T_{p} M, \omega_{\mu}\right) \simeq\left(T_{p} M, \omega\right)$ as symplectic representations for all $\mu \in(0,1]$ and all $p \in M^{T}$. But by Lemma 5.14 , for any sufficiently small $\mu>0,\left(T_{p} M, \omega_{\mu}\right) \simeq\left(T_{p} \widehat{M}_{p},\left.\omega\right|_{\widehat{M}_{p}}\right) \oplus\left(T_{\pi(p)} \widetilde{M}, \widetilde{\omega}\right)$ for all $p \in M^{T}$.

Remark 5.3. Let $\pi: M \rightarrow \widetilde{M}$ be any equivariant fiber bundle with symplectic fibers. Then $\left(T_{p} M, \omega\right) \simeq\left(T_{p} \widehat{M}_{p},\left.\omega\right|_{\widehat{M}_{p}}\right) \oplus\left(H_{p},\left.\omega\right|_{H_{p}}\right)$ for all $p \in M^{T}$, where $H_{p} \subset T_{p} M$ is the symplectic perpendicular to $T_{p} \widehat{M}_{p}$. Moreover, $d \pi: H_{p} \rightarrow T_{\pi(p)} \widetilde{M}$ is an equivariant isomorphism, and so the weights in the symplectic representations $\left(H_{p},\left.\omega\right|_{H_{p}}\right)$ and $\left(T_{\pi(p)} \widetilde{M}, \widetilde{\omega}\right)$ necessarily agree up to sign. The map $\pi$ is a strong symplectic fibration if they agree exactly.

Definition 5.4 Let $\left\{\left(M_{j}, \omega_{j}, \psi_{j}\right)\right\}_{j=0}^{k}$ be Hamiltonian $T$-manifolds with discrete-fixed sets and let $\left\{\rho_{j}: M_{j+1} \rightarrow M_{j}\right\}_{j=0}^{k-1}$ be strong symplectic fibrations. Assume that $M_{0}$ is a point. Then, given a subring $A \subseteq \mathbb{R}, M_{k}$ is a tower of complex projective spaces over $\mathbf{A}$ if the fiber $F_{j}$ of $\rho_{j}$ satisfies $H^{*}\left(F_{j} ; A\right) \simeq H^{*}\left(\mathbb{C P}^{\frac{1}{2} \operatorname{dim}\left(F_{j}\right)} ; A\right)$ as rings for all $j$.

\footnotetext{
${ }^{2}$ Every map satisfying (1) is a symplectic fibration; see [MS, Lemma 6.2].
} 
Notation: Given a strong symplectic fibration $\pi: M \rightarrow \widetilde{M}$ and a generic component of the moment map $\varphi=\psi^{\xi}: M \rightarrow \mathbb{R}$, let $\widehat{\Lambda}_{p}^{-}$denote the equivariant Euler class of the negative normal bundle of the restriction $\left.\varphi\right|_{\widehat{M}_{p}}$ at $p \in \widehat{M}_{p}$, and let $2 \widehat{\lambda}(p)$ denote the index of $p$ in $\widehat{M}_{p}$, for all $p \in M^{T}$. (Since the restriction of $\omega$ to $\widehat{M}_{p}$ is symplectic, the restriction of $\psi$ to $\widehat{M}_{p}$ is a moment map.) Similarly, let $\widetilde{\Lambda}_{q}^{-}$denote the equivariant Euler class of the negative normal bundle of $\widetilde{\varphi}=\widetilde{\psi}^{\xi}$ at $q$ and let $2 \widetilde{\lambda}(q)$ denote the index of $q \in \widetilde{M}$, for all $q \in \widetilde{M}^{T}$.

Finally, given a subring $A \subseteq \mathbb{R}$, let $A_{+}=\{t \in A \mid t>0\}$ and let $A^{\times} \subset A$ denote the set of units.

We can now state our main theorem in this section.

Theorem 5.5. Let $\left\{\left(M_{j}, \omega_{j}, \psi_{j}\right)\right\}_{j=0}^{k}$ be Hamiltonian T-manifolds with discrete-fixed sets and let $\left\{\rho_{j}: M_{j+1} \rightarrow M_{j}\right\}_{j=0}^{k-1}$ be strong symplectic fibrations. Let $\varphi_{k}=\psi_{k}^{\xi}$ be a generic component of the moment map. Fix a subring $A \subseteq \mathbb{R}$. Assume that $M_{0}$ is a point and that canonical classes $\alpha_{p} \in$ $H_{T}^{2 \lambda(p)}\left(M_{k} ; A\right)$ exist for all $p \in M_{k}^{T}$. Let $^{3} \pi_{j}=\rho_{j} \circ \rho_{j+1} \circ \cdots \circ \rho_{k-1}: M_{k} \rightarrow M_{j}$ and let $\bar{\psi}_{j}=\pi_{j}^{*}\left(\psi_{j}\right): M_{k} \rightarrow \mathfrak{t}^{*}$ for all $j$. Finally, define

$$
h\left(r, r^{\prime}\right)=\min \left\{j \in\{1, \ldots, k\} \mid \pi_{j}(r) \neq \pi_{j}\left(r^{\prime}\right)\right\} \text { for all distinct } r, r^{\prime} \text { in } M^{T} .
$$

1. Given $p$ and $q$ in $M_{k}^{T}$, let $\Sigma(p, q)$ denote the set of paths from $p$ to $q$ in the associated canonical graph $(V, E)$; then

$$
\begin{gathered}
\alpha_{p}(q)=\sum_{\gamma \in C(p, q)} \Xi(\gamma), \text { where } \\
\Xi(\gamma)=\Lambda_{q}^{-} \prod_{i=1}^{|\gamma|} \frac{\bar{\psi}_{h\left(\gamma_{i}, \gamma_{i+1}\right)}\left(\gamma_{i+1}\right)-\bar{\psi}_{h\left(\gamma_{i}, \gamma_{i+1}\right)}\left(\gamma_{i}\right)}{\bar{\psi}_{h\left(\gamma_{i}, \gamma_{i+1}\right)}(q)-\bar{\psi}_{h\left(\gamma_{i}, \gamma_{i+1}\right)}\left(\gamma_{i}\right)} \frac{\alpha_{\gamma_{i}}\left(\gamma_{i+1}\right)}{\Lambda_{\gamma_{i+1}}^{-}} \forall \gamma \in C(p, q), \text { and } \\
C(p, q)=\left\{\gamma=\left(\gamma_{1}, \ldots, \gamma_{|\gamma|+1}\right) \in \Sigma(p, q) \mid h\left(\gamma_{1}, \gamma_{2}\right) \leq \cdots \leq h\left(\gamma_{|\gamma|}, \gamma_{|\gamma|+1}\right)\right\} .
\end{gathered}
$$

2. Assume that $M_{k}$ is a tower of complex projective spaces over $A$. Then for each path $\gamma \in C(p, q)$,

- $\Xi(\gamma)$ can be written as the product of positive weights in $\ell^{*}$ and a constant $C$ in $A$; moreover, $C>0$ if $\alpha_{r}\left(r^{\prime}\right)$ is positive for all $\left(r, r^{\prime}\right) \in E$.

- If $(M, \omega, \psi)$ is a GKM space, then $\Xi(\gamma)$ can be written as the product of distinct positive weights in $\Pi_{q}(M)$ and a constant $C$ in A. Finally, if

\footnotetext{
${ }^{3}$ In this paper, our convention is that an empty composition or product is the identity. Hence, $\pi_{k}=\operatorname{id}_{M_{k}}$.
} 
$\Theta\left(r, r^{\prime}\right)>0$ for all $\left(r, r^{\prime}\right) \in E$, then $C>0$; similarly, if $\Theta\left(r, r^{\prime}\right) \in A^{\times}$ for all $\left(r, r^{\prime}\right) \in E$, then $C \in A^{\times}$.

Remark 5.6. In fact, if $M_{k}$ is a GKM space, then our proof demonstrates that claim (1) holds whenever $\rho_{j}: M_{j+1} \rightarrow M_{j}$ is a weight preserving map for all $j$; (see Definition 5.10).

Remark 5.7. If $M_{k}$ has a discrete-fixed set (or is a GKM space), then $M_{j}$ has a discrete-fixed set (or is a GKM space) for all $j$. To see this, consider any $q \in M_{j}^{T}$. Since the fiber $\rho_{j}^{-1}(q)$ is a Hamiltonian $T$-manifold, there exists $r \in M_{j+1}^{T}$ such that $\rho_{j}(r)=q$. Since the differential $d \rho_{j}$ is surjective, the set of weights in the representation $T_{q} M_{j}$ is a subset of the set of weights in $T_{r} M_{j+1}$.

Theorem 5.5 has the following useful corollary.

Corollary 5.8. Let $(M, \omega, \psi)$ and $(\widetilde{M}, \widetilde{\omega}, \widetilde{\psi})$ be Hamiltonian T-manifolds with discrete-fixed sets, and let $\pi: M \rightarrow \widetilde{M}$ be a strong symplectic fibration. Let $\varphi=\psi^{\xi}$ be a generic component of the moment map. Assume that canonical classes $\alpha_{p} \in H_{T}^{2 \lambda(p)}(M ; A)$ exist for all $p \in M^{T}$. Fix $p$ and $q$ in $M^{T}$.

1. There exist canonical classes $\widehat{\alpha}_{s} \in H_{T}^{2 \widehat{\lambda}(s)}\left(\widehat{M}_{q} ; A\right)$ on the fiber $\widehat{M}_{q}=$ $\pi^{-1}(\pi(q))$ for all $s \in \widehat{M}_{q}^{T}$.

2. Given $s \in \widehat{M}_{q}^{T}$, let $\bar{\Sigma}(p, s)$ denote the set of paths $\gamma=\left(\gamma_{1}, \ldots, \gamma_{k+1}\right)$ from $p$ to $s$ in the associated canonical graph $(V, E)$ such that $\pi\left(\gamma_{i}\right) \neq \pi\left(\gamma_{i+1}\right)$ for all $i$. Then

$$
\alpha_{p}(q)=\sum_{s \in \widehat{M}_{q}^{T}}\left(\sum_{\gamma \in \bar{\Sigma}(p, s)} P(\gamma)\right) \widehat{\alpha}_{s}(q),
$$

where for all $s \in \widehat{M}_{q}^{T}$ and $\gamma \in \bar{\Sigma}(p, s)$,

$$
P(\gamma)=\widetilde{\Lambda}_{\pi(s)}^{-} \prod_{i=1}^{|\gamma|} \frac{\widetilde{\psi}\left(\pi\left(\gamma_{i+1}\right)\right)-\widetilde{\psi}\left(\pi\left(\gamma_{i}\right)\right)}{\widetilde{\psi}(\pi(s))-\widetilde{\psi}\left(\pi\left(\gamma_{i}\right)\right)} \frac{\alpha_{\gamma_{i}}\left(\gamma_{i+1}\right)}{\Lambda_{\gamma_{i+1}}^{-}} .
$$

3. Assume that $H^{*}(\widetilde{M} ; A) \simeq H^{*}\left(\mathbb{C P}^{\frac{1}{2} \operatorname{dim}(\widetilde{M})} ; A\right)$ as rings. Then for all $s \in$ $\widehat{M}_{q}^{T}$ and each path $\gamma \in \bar{\Sigma}(p, s)$

- $P(\gamma)$ can be written as the product of positive weights in $\ell^{*}$ and a constant $C$ in $A$; moreover, $C>0$ if $\alpha_{r}\left(r^{\prime}\right)$ is positive for all $\left(r, r^{\prime}\right) \in E$.

- If $(M, \omega, \psi)$ is a GKM space, then $P(\gamma)$ can be written as the product of distinct positive weights in $\Pi_{q}(M)$ and a constant $C$ in A. Finally, if $\Theta\left(r, r^{\prime}\right)>0$ for all $\left(r, r^{\prime}\right) \in E$, then $C>0$; similarly, if $\Theta\left(r, r^{\prime}\right) \in A^{\times}$ for all $\left(r, r^{\prime}\right) \in E$, then $C \in A^{\times}$. 
In Lemma 5.24, we give a different explicit description of $P(\gamma)$ in the case that $(M, \omega, \psi)$ is a GKM space and $H^{*}(\widetilde{M} ; A) \simeq H^{*}\left(\mathbb{C P}^{\frac{1}{2} \operatorname{dim} \widetilde{M}} ; A\right)$.

Proof of Claim 1 of Theorem 5.5. We are now ready to begin the proof of the first part of our main theorem. We will begin with the special case of GKM spaces, where the proof is easier and the main ideas are more transparent. However, the proof in the general case on page 198 is selfcontained; the reader may skip directly to that case.

The case of GKM spaces. Let $(M, \omega, \psi)$ and $(\widetilde{M}, \widetilde{\omega}, \widetilde{\psi})$ be GKM spaces, and let $\left(V, E_{\mathrm{GKM}}\right)$ and $\left(\widetilde{V}, \widetilde{E}_{\mathrm{GKM}}\right)$ be the associated GKM graphs. If $\pi: M \rightarrow$ $\widetilde{M}$ is an equivariant map, the following statements hold:

- Given a vertex $p \in V, \pi(p) \in \widetilde{V}$.

- Given an edge $e=(p, q) \in E_{\mathrm{GKM}}$, either $\pi(p)=\pi(q) \in \widetilde{V}$ or $\pi(e)=$ $(\pi(p), \pi(q)) \in \widetilde{E}_{\mathrm{GKM}}$ and $\eta(\pi(e))$ is a non-zero multiple of $\eta(e)$.

To see this, let $K \subset T$ be the maximal subgroup so that $p$ and $q$ are contained in the same connected component $N \subset M^{K}$. Since $\pi$ is equivariant, either $\pi(N)$ is a fixed point in $\widetilde{M}$, or $\pi(N)$ is the connected component of $\widetilde{M}^{K^{\prime}}$ for some subgroup $K^{\prime} \varsubsetneqq T$ which contains $K$.

Definition 5.9 We will say that an edge $(p, q) \in\left(V, E_{\mathrm{GKM}}\right)$ is horizontal (with respect to $\pi$ ) if $\pi(p) \neq \pi(q)$; moreover, we will say that a path $\gamma$ in $\left(V, E_{\mathrm{GKM}}\right)$ is horizontal if all its edges are horizontal.

If $\pi: M \rightarrow \widetilde{M}$ is an equivariant fiber bundle and $e \in E_{\mathrm{GKM}}$ is a horizontal edge, then $\eta(e)= \pm \eta(\pi(e))$. However, this need not hold for arbitrary equivariant maps.

Definition 5.10 We will say that a map $\pi: M \rightarrow \widetilde{M}$ is weight preserving if it is equivariant and $\eta(e)=\eta(\pi(e))$ for all horizontal edges $(p, q) \in E_{\mathrm{GKM}}$.

Note that the composition of two weight preserving maps is itself weight preserving. In contrast, the composition of two strong symplectic fibrations may not be a strong symplectic fibration; indeed, it may not have symplectic fibers. However, the following assertion is clear; cf. Remark 5.3.

Lemma 5.11. Let $(M, \omega, \psi)$ and $(\widetilde{M}, \widetilde{\omega}, \widetilde{\psi})$ be GKM spaces. If $\pi: M \rightarrow \widetilde{M}$ is a strong symplectic fibration then $\pi$ is weight preserving.

To prove Claim 1., we need to check that the pull-back of a symplectic form and moment map by a weight preserving map satisfies criterion (1) of Corollary 3.2. We will do this in two steps.

Lemma 5.12. Let $(M, \omega, \psi)$ and $(\widetilde{M}, \widetilde{\omega}, \widetilde{\psi})$ be GKM spaces, and let $\pi: M \rightarrow$ $\widetilde{M}$ be a weight preserving map. Let $\varphi=\psi^{\xi}$ be a generic component of the 
moment map. Given a horizontal edge $(p, q)$ in the GKM graph associated to $M$,

$$
\psi^{\xi}(q)-\psi^{\xi}(p)>0, \quad \text { if and only if } \widetilde{\psi}^{\xi}(\pi(q))-\widetilde{\psi}^{\xi}(\pi(p))>0 .
$$

Proof. Since $(p, q)$ is a horizontal edge and $\pi$ is a weight preserving map, $\eta(\pi(p), \pi(q))=\eta(p, q)$. Therefore, $\psi^{\xi}(q)-\psi^{\xi}(p)$ and $\widetilde{\psi}^{\xi}(\pi(q))-\widetilde{\psi}^{\xi}(\pi(p))$ are both positive multiples of $\eta(p, q)$.

Lemma 5.13. Let $(M, \omega, \psi)$ and $(\widetilde{M}, \widetilde{\omega}, \widetilde{\psi})$ be GKM spaces, and let $\pi: M \rightarrow$ $\widetilde{M}$ be a weight preserving map. Let $\varphi=\psi^{\xi}$ be a generic component of the moment map. Given a canonical class $\alpha_{p} \in H_{T}^{2 \lambda(p)}(M ; A)$ at $p \in M^{T}$,

$\alpha_{p}(q)=0 \quad$ for all $q \in M^{T}$ such that $\pi(q) \neq \pi(p)$ and $\widetilde{\psi}^{\xi}(\pi(q)) \leq \widetilde{\psi}^{\xi}(\pi(p))$.

Proof. Assume that $\alpha_{p}(q) \neq 0$ for some $q \in M^{T}$. By Lemma 2.3, there exists an ascending path $\gamma$ from $p$ to $q$ in $\left(V, E_{\mathrm{GKM}}\right)$. By Lemma 5.12 and the definition of ascending,

$$
\widetilde{\psi}^{\xi}\left(\pi\left(\gamma_{i}\right)\right)<\widetilde{\psi}^{\xi}\left(\pi\left(\gamma_{i+1}\right)\right) \quad \text { or } \quad \pi\left(\gamma_{i}\right)=\pi\left(\gamma_{i+1}\right) \quad \text { for each } i .
$$

We are now ready to prove Claim 1 . Let $\mathrm{w}_{j}=\pi_{j}^{*}\left(\omega_{j}+\psi_{j}\right) \in H_{T}^{2}\left(M_{k} ; \mathbb{R}\right)$ for each $j \in\{1, \ldots, k\}$. Since $\pi_{k}=\mathrm{id}_{M_{k}}$, it is obvious that $\mathrm{w}_{k}(r) \neq \mathrm{w}_{k}\left(r^{\prime}\right)$ for all $\left(r, r^{\prime}\right) \in E_{\mathrm{GKM}}$. By Lemma 5.11, each $\rho_{i}$ is a weight preserving map, and so $\pi_{j}$ is a weight preserving map for all $j$. Therefore, in the case of GKM spaces, Claim 1. of Theorem 5.5 is an immediate consequence of Corollary 3.2 and Lemma 5.13.

The general case. The proof in the general case is nearly identical, except that it takes more work to prove Lemma 5.16, the analog of Lemma 5.13.

Lemma 5.14. Let $(M, \omega, \psi)$ and $(\widetilde{M}, \widetilde{\omega}, \widetilde{\psi})$ be Hamiltonian T-manifolds, and let $\pi: M \rightarrow \widetilde{M}$ be a equivariant fiber bundle with symplectic fibers. For all sufficiently small $t>0$,

(1) the two-form $\omega_{t}=\pi^{*}(\widetilde{\omega})+t \omega$ is symplectic; moreover,

(2) as symplectic representations $\left(T_{p} M, \omega_{t}\right) \simeq\left(T_{p} \widehat{M}_{p}, \omega{\widehat{M_{p}}}_{p}\right) \oplus\left(T_{\pi(p)} \widetilde{M}, \widetilde{\omega}\right)$ for all $p \in M^{T}$, where $\widehat{M}_{p}$ denotes the fiber $\pi^{-1}(\pi(p))$.

Proof. Let $V \subset T M$ be the kernel of the map $d \pi: T M \rightarrow T \widetilde{M}$. By assumption, $\pi$ is a submersion; hence, $V \subset T M$ is a subbundle. Since we have assumed that $\pi$ has symplectic fibers, the restriction $\left.\omega\right|_{V}$ is symplectic. Since $\left.\pi^{*}(\widetilde{\omega})\right|_{V}=0$, this implies that the restriction $\left.\omega_{t}\right|_{V}=\left.t \omega\right|_{V}$ is symplectic and that $\left(V_{p}, \omega_{t}\right) \simeq\left(V_{p}, \omega\right)$ for all $p \in M^{T}$ and $t>0$. 
Let $H=V^{\omega} \subset T M$ be the symplectic perpendicular to $V$ with respect to $\omega$. Since $\left.\pi^{*}(\widetilde{\omega})\right|_{V}=0, H$ is also symplectically perpendicular to $V$ with respect to $\omega_{t}$ for all $t \geq 0$. Moreover, since $\left.\omega\right|_{V}$ is symplectic, $H \subset T M$ is a subbundle and $T M=V \oplus H$. Thus, the map $d \pi: H \rightarrow T \widetilde{M}$ is an isomorphism. Since $\widetilde{\omega}$ is symplectic, this implies that the restriction $\left.\pi^{*}(\widetilde{\omega})\right|_{H}$ is symplectic and that $\left(H_{p},\left.\pi^{*}(\widetilde{\omega})\right|_{H_{p}}\right) \simeq\left(T_{\pi(p)} \widetilde{M}, \widetilde{\omega}\right)$ for all $p \in M^{T}$. Since being symplectic is an open condition and $M$ is compact, analogous statements hold for $\omega_{t}$ for all sufficiently small $t>0$. The claim follows immediately.

Lemma 5.15. Let $(M, \omega, \psi)$ be a Hamiltonian T-manifold with discretefixed set. Let $\varphi=\psi^{\xi}$ be a generic component of the moment map, and let $\bar{\varphi}: M \rightarrow \mathbb{R}$ be an invariant Morse-Bott function. Assume that for all $\epsilon>0$ there exists a symplectic form $\omega^{\prime} \in \Omega^{2}(M)$ with moment map $\psi^{\prime}$ such that:

(a) $\left|\left(\psi^{\prime}\right)^{\xi}(x)-\bar{\varphi}(x)\right|<\epsilon$ for all $x \in M$; and

(b) the product of the positive weights for the isotropy action of $T$ on $\left(T_{p} M, \omega^{\prime}\right)$ is $\Lambda_{p}^{-}$for all $p \in M^{T}$.

If $\alpha_{p} \in H_{T}^{2 \lambda(p)}(M ; A)$ is the canonical class (with respect to $\varphi$ ) at $p \in M^{T}$, and $\widehat{M}_{p}$ is the critical component of $\bar{\varphi}$ that contains $p$, then

$$
\alpha_{p}(q)=0 \quad \text { for all } q \in M^{T} \text { so that } q \notin \widehat{M}_{p} \text { and } \bar{\varphi}(q) \leq \bar{\varphi}(p) \text {. }
$$

Moreover, the restriction of $\alpha_{p}$ to $\bar{\varphi}^{-1}(-\infty, \bar{\varphi}(p)-\delta)$ vanishes for all $\delta>0$.

Proof. We may assume without loss of generality that $\bar{\varphi}(p)=0$. By assumption, for any $\epsilon>0$ there exists a symplectic form $\omega^{\prime}$ with moment map $\psi^{\prime}$ such that (a) and (b) hold. Let $\varphi^{\prime}$ be $\left(\psi^{\prime}\right)^{\xi}$. By (b) it follows that $\alpha_{p}$ is also the canonical class at $p$ with respect to $\varphi^{\prime}$; so Lemma 1.1 implies that

$$
\alpha_{p}(q)=0 \quad \text { for all } q \in M^{T} \text { such that } \varphi^{\prime}(q)<\varphi^{\prime}(p) .
$$

By injectivity, this implies that the restriction of $\alpha_{p}$ to $\left(\varphi^{\prime}\right)^{-1}\left(-\infty, \varphi^{\prime}(p)\right)$ vanishes. Finally, (a) implies that

$$
(\bar{\varphi})^{-1}(-\infty,-2 \epsilon) \subset\left(\varphi^{\prime}\right)^{-1}\left(-\infty, \varphi^{\prime}(p)\right),
$$

and so the restriction of $\alpha_{p}$ to $\bar{\varphi}^{-1}(-\infty,-2 \epsilon)$ vanishes.

Since $\bar{\varphi}$ is a Morse-Bott function there exists $\epsilon>0$ so that 0 is the only critical value of $\bar{\varphi}$ in $[-2 \epsilon, 2 \epsilon]$. Since the restriction of $\alpha_{p}$ to $\bar{\varphi}^{-1}(-\infty,-2 \epsilon)$ vanishes, the restriction of $\alpha_{p}$ to $\widehat{M}_{p}$ is a multiple of the equivariant Euler class of the negative normal bundle of $\bar{\varphi}$ at $\widehat{M}_{p}$, and so there exists $\alpha_{p}^{\prime} \in$ $H_{T}^{2 \lambda(p)}\left((\bar{\varphi})^{-1}(-\infty, 2 \epsilon) ; A\right)$ so that $\left.\alpha_{p}^{\prime}\right|_{\widehat{M}_{p}}=\left.\alpha_{p}\right|_{\widehat{M}_{p}}$, but $\left.\alpha_{p}^{\prime}\right|_{C}=0$ for every 
other critical set $C$ of $\bar{\varphi}$ so that $\bar{\varphi}(C) \leq 2 \epsilon$. Moreover, since $\bar{\varphi}$ is invariant and the fixed set is discrete, every fixed point is critical. Hence,

$$
\begin{aligned}
& \alpha_{p}^{\prime}(q)=\alpha_{p}(q) \text { for all } q \in \widehat{M}_{p} \cap M^{T}, \quad \text { and } \\
& \alpha_{p}^{\prime}(q)=0 \quad \text { for all } q \in M^{T} \text { such that } q \notin \widehat{M}_{p} \text { and } \bar{\varphi}(q) \leq 2 \epsilon .
\end{aligned}
$$

By $\left(\right.$ a), $\left(\varphi^{\prime}\right)^{-1}(-\infty, \epsilon) \subset(\bar{\varphi})^{-1}(-\infty, 2 \epsilon)$. Hence, we can restrict $\alpha_{p}^{\prime}$ to $\left(\varphi^{\prime}\right)^{-1}(-\infty, \epsilon)$; moreover, this restriction satisfies (4) and

$$
\alpha_{p}^{\prime}(q)=0 \quad \text { for all } q \in M^{T} \text { such that } q \notin \widehat{M}_{p} \text { and } \varphi^{\prime}(q)<\epsilon .
$$

By surjectivity, we can extend $\alpha_{p}^{\prime}$ to a class (which we still call $\alpha_{p}^{\prime}$ ) on $M$ with the same properties. Moreover, by the definition of canonical class,

$$
\alpha_{p}(q)=0 \text { for all } q \in M^{T} \backslash\{p\} \text { such that } \lambda(q) \leq \lambda(p) .
$$

Therefore, by (4) and (6),

$$
\alpha_{p}(q)=\alpha_{p}^{\prime}(q) \text { for all } q \in M^{T} \text { such that } \varphi^{\prime}(q)<\epsilon \text { and } \lambda(q) \leq \lambda(p) .
$$

Assume that there exists $r \in M^{T}$ such that $\alpha_{p}(r) \neq \alpha_{p}^{\prime}(r)$ and $\varphi^{\prime}(r)<\epsilon$ but $\alpha_{p}(s)=\alpha_{p}^{\prime}(s)$ for all $s \in M^{T}$ such that $\varphi^{\prime}(s)<\varphi^{\prime}(r)$. By the equation above, this implies that $\lambda(r)>\lambda(p)$. Since $\beta=\alpha_{p}-\alpha_{p}^{\prime}$ has degree $2 \lambda(p)$, this contradicts Lemma 1.3. Therefore,

$$
\alpha_{p}(q)=\alpha_{p}^{\prime}(q) \text { for all } q \in M^{T} \text { such that } \varphi^{\prime}(q)<\epsilon .
$$

Finally, since $(\bar{\varphi})^{-1}((-\infty, 0]) \subset\left(\varphi^{\prime}\right)^{-1}(-\infty, \epsilon)$ by $(\mathrm{a})$, this implies that

$$
\alpha_{p}(q)=\alpha_{p}^{\prime}(q) \text { for all } q \in M^{T} \text { such that } \bar{\varphi}(q) \leq 0 .
$$

Therefore, the claim follows immediately from (5).

Lemma 5.16. Let $\left\{\left(M_{j}, \omega_{j}, \psi_{j}\right)\right\}_{j=1}^{k}$ be Hamiltonian T-manifolds with discrete-fixed sets and let $\left\{\rho_{j}: M_{j+1} \rightarrow M_{j}\right\}_{j=1}^{k-1}$ be strong symplectic fibrations. Let $\varphi_{k}=\psi_{k}^{\xi}$ be a generic component of the moment map. Let $\pi=\rho_{1} \circ \rho_{2} \circ \cdots \circ \rho_{k-1}: M_{k} \rightarrow M_{1}$. Given a canonical class $\alpha_{p} \in H_{T}^{2 \lambda(p)}\left(M_{k} ; A\right)$ at $p \in M_{k}^{T}$,

$$
\alpha_{p}(q)=0 \text { for all } q \in M_{k}^{T} \text { so that } \pi(q) \neq \pi(p) \text { and } \psi_{1}^{\xi}(\pi(q)) \leq \psi_{1}^{\xi}(\pi(p)) .
$$

Proof. Given $q \in M_{k}^{T}$, let $q_{j}=\left(\rho_{j} \circ \rho_{j+1} \circ \cdots \circ \rho_{k-1}\right)(q) \in M_{j}$ for all $j$, let $X_{j}=\rho_{j-1}^{-1}\left(q_{j-1}\right) \subset M_{j}$ for $j>1$, and let $X_{1}=M_{1}$. By the definition of 
strong symplectic fibration and induction on $k$, as symplectic representations

$$
\left(T_{q} M_{k}, \omega_{k}\right) \simeq\left(T_{q_{1}} X_{1},\left.\omega_{1}\right|_{X_{1}}\right) \oplus \cdots \oplus\left(T_{q_{k}} X_{k},\left.\omega_{k}\right|_{X_{k}}\right) .
$$

Therefore, by Lemma 5.14 and induction on $k$, for any $\epsilon>0$ there exists a symplectic form $\omega_{k}^{\prime} \in \Omega^{2}\left(M_{k}\right)$ with moment map $\psi_{k}^{\prime}$ such that:

- $\left|\left(\psi_{k}^{\prime}\right)^{\xi}(x)-\pi^{*}\left(\psi_{1}\right)^{\xi}(x)\right|<\epsilon$ for all $x \in M_{k}$; and

- as symplectic representations $\left(T_{q} M_{k}, \omega_{k}\right) \simeq\left(T_{q} M_{k}, \omega_{k}^{\prime}\right)$ for all $q \in M_{k}^{T}$.

Since $M_{1}$ has a discrete-fixed set, $\psi_{1}^{\xi}: M_{1} \rightarrow \mathbb{R}$ is a Morse function on $M_{1}$ with critical set $M_{1}^{T}$. Since $\pi$ is an equivariant fiber bundle, this implies that $\pi^{*}\left(\psi_{1}\right)^{\xi}$ is an invariant Morse-Bott function on $M$, and that the critical component of $\pi^{*}\left(\psi_{1}\right)^{\xi}$ that contains $p \in M_{k}^{T}$ is the fiber $\pi^{-1}(\pi(p))$. Therefore, the claim follows from Lemma 5.15.

We are now ready to prove Claim 1 . Let $\mathrm{w}_{j}=\left[\pi_{j}^{*}\left(\omega_{j}+\psi_{j}\right)\right] \in H_{T}^{2}\left(M_{k} ; \mathbb{R}\right)$ for each $j \in\{1, \ldots, k\}$. Since $\pi_{k}=\operatorname{id}_{M_{k}}$, Lemma 1.1 implies that $\mathrm{w}_{k}(r) \neq$ $\mathrm{w}_{k}\left(r^{\prime}\right)$ for all $\left(r, r^{\prime}\right) \in E$. Therefore, Claim 1. of Theorem 5.5 is an immediate consequence of Corollary 3.2 and Lemma 5.16.

Proof of Claim 2. of Theorem 5.5. Let a maximal subtorus $T \subset S U(n+$ $1)$ act on $\left(\mathbb{C P}^{n}, \omega\right)$, and let $\varphi$ be a generic component of the moment map $\psi: \mathbb{C P}^{n} \rightarrow \mathfrak{t}^{*}$. If $[\omega]$ generates $H^{2}\left(\mathbb{C P}^{n} ; \mathbb{Z}\right)$ then

$$
\Lambda_{p}^{-}=\prod_{\varphi(y)<\varphi(p)} \psi(p)-\psi(y)
$$

where the sum is over all $y \in\left(\mathbb{C P}^{n}\right)^{T}$ such that $\varphi(y)<\varphi(p)$. The next lemma, which is the key ingredient in the proof of Claim 2. of Theorem 5.5 and Claim 3. of Corollary 5.8, generalizes this fact to other manifolds with isomorphic cohomology rings.

Definition 5.17 Fix a GKM space $(M, \omega, \psi)$ with GKM graph $\left(V, E_{\mathrm{GKM}}\right)$. The magnitude of an edge $(r, s) \in E_{\mathrm{GKM}}$ is

$$
m(r, s)=\frac{\psi(s)-\psi(r)}{\eta(r, s)} .
$$

Lemma 5.18. Let $(M, \omega, \psi)$ be a Hamiltonian T-manifold with discrete-fixed set, and let $\varphi=\psi^{\xi}$ be a generic component of the moment map. Assume that $[\omega]$ generates $H^{2}(M ; \mathbb{Z})$ and that $H^{*}(M ; A) \simeq H^{*}\left(\mathbb{C P}^{\frac{1}{2} \operatorname{dim} M} ; A\right)$ as rings. Given $p \in M^{T}$, fix a subset $S \subset\left\{y \in M^{T} \mid \varphi(y)<\varphi(p)\right\}$. Then

- $\Lambda_{p}^{-} \prod_{y \in S} \frac{1}{\psi(p)-\psi(y)}$ can be written as the product of positive weights in $\ell^{*}$ and a constant in $A_{+}$. 
- If $(M, \omega, \psi)$ is a GKM space with $G K M$ graph $\left(V, E_{\mathrm{GKM}}\right)$, then $m(r, s)$ is a unit in $A_{+}$for all $(r, s) \in E_{\mathrm{GKM}}$ such that $\varphi(r)<\varphi(s)$. In particular $\Lambda_{p}^{-} \prod_{y \in S} \frac{1}{\psi(p)-\psi(y)}$ can be written as the product of distinct positive weights in $\Pi_{p}(M)$ and a unit in $A_{+}$.

Proof. Since the fixed set is discrete and $\varphi$ is a perfect Morse function, there is exactly one fixed point of index $2 i$ for all $i \in\left\{0, \ldots, \frac{1}{2} \operatorname{dim}(M)\right\}$. Therefore, there are exactly $\lambda(p)$ fixed points $y$ with $\lambda(y)<\lambda(p)$. Moreover, by $[\mathbf{L T}$, Lemma 2.7], the fact that $[\omega]$ is integral implies that $[\omega+\psi-\psi(y)] \in$ $H_{T}^{2}(M ; \mathbb{Z})$ for all $y \in M^{T}$. Therefore, we may define a class

$$
\beta=\prod_{\lambda(y)<\lambda(p)}[\omega+\psi-\psi(y)] \in H_{T}^{2 \lambda(p)}(M ; \mathbb{Z}),
$$

where the product is over all $y \in M^{T}$ such that $\lambda(y)<\lambda(p)$.

Since $H^{2 i}(M ; \mathbb{R})=H^{2 i}\left(\mathbb{C P}^{\frac{1}{2} \operatorname{dim}(M)} ; \mathbb{R}\right)$ for all $i,[\mathbf{T}$, Proposition 3.4] (and the fact that rational $\xi \in \mathfrak{t}$ are dense) implies that

$$
\varphi(y)<\varphi(p) \quad \text { exactly if } \lambda(y)<\lambda(p) \quad \text { for all } y \in M^{T} .
$$

Since $\beta(y)=0$ for all $y \in M^{T}$ such that $\lambda(y)<\lambda(p)$, Lemma 1.2, Corollary 1.3, and (7) together imply that we can write

$$
\beta=\sum_{\lambda(y) \geq \lambda(p)} x_{y} \gamma_{y}
$$

where the sum is over $y \in M^{T}$ such that $\lambda(y) \geq \lambda(p), \gamma_{y} \in H_{T}^{2 \lambda(y)}(M ; \mathbb{Z})$, $x_{y} \in H^{2 \lambda(p)-2 \lambda(y)}(B T ; \mathbb{Z})$ for all $y \in M^{T}$, and $\left\{\gamma_{y}\right\}_{y \in M^{T}}$ is a basis for $H_{T}^{*}(M ; \mathbb{Z})$ as a $H^{*}(B T ; \mathbb{Z})$ module. Since $p$ is the only fixed point with index $2 \lambda(p)$, by degree considerations this implies that

$$
\beta=x_{p} \gamma_{p}, \quad \text { where } x_{p} \in \mathbb{Z} .
$$

Since $[\omega]^{\lambda(p)}$ is the image of $\beta$ under the natural restriction map from $H_{T}^{*}(M ; \mathbb{Z})$ to $H^{*}(M ; \mathbb{Z})$, this implies that

$$
[\omega]^{\lambda(p)}=x_{p} \widetilde{\gamma}_{p} \text { for all } p \in M^{T},
$$

where $\widetilde{\gamma}_{p}$, the restriction of $\gamma_{p}$, generates $H^{2 \lambda(p)}(M ; \mathbb{Z})$. Moreover, since we have assumed that $[\omega]$ generates $H^{2}(M ; \mathbb{Z})$ and that $H^{*}(M ; A) \simeq$ $H^{*}\left(\mathbb{C P}^{\frac{1}{2} \operatorname{dim} M} ; A\right)$ as rings, $[\omega]^{\lambda(p)}$ generates $H^{2 \lambda(p)}(M ; A)$. Hence, the equation above implies that $x_{p}$ must be invertible in $A$. Therefore, evaluating both sides of (8) at $p$,

$$
\Lambda_{p}^{-} \prod_{\varphi(y)<\varphi(p)} \frac{1}{\psi(p)-\psi(y)}=\frac{1}{x_{p}} \quad \in A .
$$


Now observe that $\psi(p)-\psi(y)$ is the product of a positive integer and a positive weight in $\ell^{*}$ for all $y \in M^{T}$ such that $\varphi(y)<\varphi(p)$. This proves the first claim.

If $M$ is a GKM space, then since the GKM graph is $\frac{1}{2} \operatorname{dim}(M)$-valent and has $\frac{1}{2} \operatorname{dim}(M)+1$ vertices, it is a complete graph. Therefore $\psi(p)-\psi(y)=$ $m(y, p) \eta(y, p)$ for all $y \in M^{T}$; moreover $m(y, p) \in \mathbb{Z}_{+}$and $\eta(y, p)$ is a positive weight in $\Pi_{p}(M)$ for all $y \in M^{T}$ such that $\varphi(y)<\varphi(p)$. By $(9)$ we have that

$$
x_{p}=\prod_{\varphi(y)<\varphi(p)} m(y, p)
$$

is a unit in $A_{+}$, which implies that $m(y, p)$ is a unit in $A_{+}$for all $y, p \in M^{T}$ such that $\varphi(y)<\varphi(p)$. Finally, observe that the weights in $\Pi_{p}(M)$ are all distinct since $M$ is a GKM space, and the second claim follows immediately. (Notice that in the GKM case (7) directly follows from the fact that the GKM graph is complete.)

Remark 5.19. Fix a GKM space $(M, \omega, \psi)$ with $\operatorname{GKM}$ graph $\left(V, E_{\mathrm{GKM}}\right)$; assume that $H^{*}(M ; \mathbb{R}) \simeq H^{*}\left(\mathbb{C} \mathbb{P}^{\frac{1}{2} \operatorname{dim} M} ; \mathbb{R}\right)$ and that $[\omega]$ generates $H^{2}$ $(M ; \mathbb{Z})$. Then by $(10)$, the magnitudes of the edges of $\left(V, E_{\mathrm{GKM}}\right)$ determine the ring structure of $H^{*}(M ; \mathbb{Z})$.

We also need a technical lemma.

Lemma 5.20. Let $(M, \omega, \psi)$ and $(\widetilde{M}, \widetilde{\omega}, \widetilde{\psi})$ be Hamiltonian T-manifolds, and let $\pi: M \rightarrow \widetilde{M}$ be a strong symplectic fibration. The natural restriction map $H_{T}^{*}(M ; \mathbb{Z}) \rightarrow H^{*}\left(\widehat{M}_{p} ; \mathbb{Z}\right)$ is surjective for all $p \in M$, where $\widehat{M}_{p}$ is the fiber $\pi^{-1}(\pi(p))$.

Proof. Let $\varphi=\psi^{\xi}$ be a generic component of the moment map. Since $\widetilde{M} \times_{T}$ $E T$ is connected and $\pi$ induces a fiber bundle $M \times_{T} E T \rightarrow \widetilde{M} \times_{T} E T$ with fiber $\widehat{M}_{p}$, we may assume that $\pi(p) \in \widetilde{M}^{T}$ is the minimal fixed point. Hence, $\widetilde{\Lambda}_{\pi(p)}^{-}=1$.

Consider any point $q \in \widehat{M}_{p}^{T}$. By Lemma 1.2, there exists a class $\gamma_{q} \in$ $H_{T}^{2 \lambda(q)}(M ; \mathbb{Z})$ such that $\gamma_{q}(q)=\Lambda_{q}^{-}$and $\gamma_{q}(r)=0$ for all $r \in M^{T} \backslash\{q\}$ such that $\varphi(r) \leq \varphi(q)$. By definition of strong symplectic fibration and the paragraph above, $\Lambda_{q}^{-}=\widetilde{\Lambda}_{\pi(q)}^{-} \widehat{\Lambda}_{q}^{-}=\widetilde{\Lambda}_{\pi(p)} \widehat{\Lambda}_{q}^{-}=\widehat{\Lambda}_{q}^{-}$. Therefore, if $\widehat{\beta}_{q}$ denotes the restriction of $\gamma_{q}$ to $\widehat{M}_{p}$, then $\widehat{\beta}_{q}(q)=\widehat{\Lambda}_{q}^{-}$, and $\widehat{\beta}_{q}(r)=0$ for all $r \in \widehat{M}_{q}^{T} \backslash\{q\}$ such that $\varphi(r) \leq \varphi(q)$. By Lemma 1.2, this implies that $\left\{\widehat{\beta}_{q}\right\}_{q \in \widehat{M}_{p}^{T}}$ is a basis for $H_{T}^{*}\left(\widehat{M}_{p} ; \mathbb{Z}\right)$ as a $H^{*}(B T ; \mathbb{Z})$ module. Hence, the map $H_{T}^{*}(M ; \mathbb{Z}) \rightarrow H_{T}^{*}\left(\widehat{M}_{p} ; \mathbb{Z}\right)$ is surjective. Finally, since the fixed set is discrete, 
$H^{*}\left(M^{T} ; \mathbb{Z}\right)$ is torsion-free, and so the natural restriction map $H_{T}^{*}\left(\widehat{M}_{p} ; \mathbb{Z}\right) \rightarrow$ $H^{*}\left(\widehat{M}_{p} ; \mathbb{Z}\right)$ is surjective; see, for example, [T, Section 2$]$.

Proof of Claim 2. of Theorem 5.5. Let $q_{j}=\pi_{j}(q)$ for all $j$, and let $X_{j}=$ $\rho_{j-1}^{-1}\left(q_{j-1}\right) \subset M_{j}$ be the fiber over $q_{j-1}$. Note that the value of $\Xi(\gamma)$ does not change if we multiply $\omega_{j}+\psi_{j}$ by a non-zero constant or add any constant to it. Moreover, by Lemma 5.20 the restriction map from $H_{T}^{2}\left(M_{j} ; \mathbb{Z}\right)$ to $H^{2}\left(X_{j} ; \mathbb{Z}\right)$ is surjective. Therefore, since $H^{2}\left(X_{j} ; \mathbb{R}\right)=\mathbb{R}$, we may assume that $\left[\omega_{j}+\psi_{j}\right]$ lies $\operatorname{in}^{4} H_{T}^{2}\left(M_{j} ; \mathbb{Z}\right)$ and that $\left[\left.\omega_{j}\right|_{X_{j}}\right]$ generates $H^{2}\left(X_{j} ; \mathbb{Z}\right)$.

Let $\widehat{\Lambda}_{q_{j}}^{-}$denote the equivariant Euler class of the negative normal bundle of $\left.\psi_{j}^{\xi}\right|_{X_{j}}$ at $q_{j} \in X_{j}$, and let $\Lambda_{q_{j}}^{-}$denote the equivariant Euler class of the negative normal bundle of $\psi_{j}^{\xi}$ at $q_{j} \in M_{j}$. By the definition of strong symplectic fibration, $\Lambda_{q_{j}}^{-}=\widehat{\Lambda}_{q_{j}}^{-} \Lambda_{q_{j-1}}^{-}$for all $j$. Since $M_{0}$ is a point, this implies by induction that

$$
\Lambda_{q}^{-}=\prod_{j=1}^{k} \widehat{\Lambda}_{q_{j}}^{-} .
$$

Therefore, to prove the claim it is enough to prove that given $h \in$ $\{1, \ldots, k\}$ such that the fiber $X_{h}$ satisfies $H^{*}\left(X_{h} ; A\right) \simeq H^{*}\left(\mathbb{C P}^{\frac{1}{2} \operatorname{dim} X_{h}} ; A\right)$ as rings, $r$ and $s$ in $M_{k}^{T}$ such that $\pi_{h}(s)=\pi_{h}(q)=q_{h}$, and a path $\gamma$ from $r$ to $s$ such that $h\left(\gamma_{i}, \gamma_{i+1}\right)=h$ for all $i \in\{1, \ldots,|\gamma|\}$, if we define

$$
\Xi_{h}(\gamma)=\widehat{\Lambda}_{q_{h}}^{-} \prod_{i=1}^{|\gamma|} \frac{\bar{\psi}_{h}\left(\gamma_{i+1}\right)-\bar{\psi}_{h}\left(\gamma_{i}\right)}{\bar{\psi}_{h}(q)-\bar{\psi}_{h}\left(\gamma_{i}\right)} \frac{\alpha_{\gamma_{i}}\left(\gamma_{i+1}\right)}{\Lambda_{\gamma_{i+1}}^{-}}
$$

then

(a1) $\Xi_{h}(\gamma)$ can be written as the product of positive weights in $\ell^{*}$ and a constant $C$ in $A$; moreover, $C>0$ if $\alpha_{r}\left(r^{\prime}\right)$ is positive for all $\left(r, r^{\prime}\right) \in E$.

(b1) If $\left(M_{k}, \omega_{k}, \psi_{k}\right)$ is a GKM space, then $\Xi_{h}(\gamma)$ can be written as the product of distinct positive weights in $\Pi_{q}(M)$ and a constant $C$ in $A$. Finally, if $\Theta\left(r, r^{\prime}\right)>0$ for all $\left(r, r^{\prime}\right) \in E$, then $C>0$; similarly, if $\Theta\left(r, r^{\prime}\right) \in A^{\times}$ for all $\left(r, r^{\prime}\right) \in E$, then $C \in A^{\times}$.

To prove this, first note that since $h\left(\gamma_{i}, \gamma_{i+1}\right)=h$ for all $i$ and $\pi_{h}(s)=$ $\pi_{h}(q), \pi_{h}\left(\gamma_{i}\right) \in X_{h}$ and $\pi_{h}\left(\gamma_{i}\right) \neq \pi_{h}\left(\gamma_{i+1}\right)$ for all $i$. So by Lemma 5.16 (or Lemmas 5.11 and 5.13 if $M_{k}$ is GKM)

$$
\bar{\psi}_{h}^{\xi}\left(\gamma_{i}\right)<\bar{\psi}_{h}^{\xi}\left(\gamma_{i+1}\right) \text { for all } 1 \leq i \leq|\gamma| \text {. }
$$

\footnotetext{
${ }^{4}$ Since the fixed set is discrete $H_{T}^{2}\left(M_{j} ; \mathbb{Z}\right)$ and $H^{2}\left(X_{j} ; \mathbb{Z}\right)$ are torsion-free. Therefore, we can identify these groups with their images in $H_{T}^{2}\left(M_{j} ; \mathbb{R}\right)$ and $H^{2}\left(X_{j} ; \mathbb{R}\right)$, respectively.
} 
Hence, $\pi_{h}\left(\gamma_{i}\right) \neq \pi_{h}\left(\gamma_{j}\right)$ for all $i \neq j$ and $\bar{\psi}_{h}^{\xi}\left(\gamma_{i}\right)<\bar{\psi}_{h}^{\xi}(s)$ for all $1 \leq$ $i \leq|\gamma|$. Therefore, since $\left[\left.\omega_{j}\right|_{X_{j}}\right]$ generates $H^{2}\left(X_{j} ; \mathbb{Z}\right)$ and $H^{*}\left(X_{h} ; A\right) \simeq$ $H^{*}\left(\mathbb{C} P^{\frac{1}{2} \operatorname{dim} X_{h}} ; A\right)$, Lemma 5.18 implies that

(a2) $\widehat{\Lambda}_{q_{h}}^{-} \prod_{i=1}^{|\gamma|} \frac{1}{\bar{\psi}_{h}(q)-\bar{\psi}_{h}\left(\gamma_{i}\right)}$ can be written as the product of positive weights in $\ell^{*}$ and a constant in $A_{+}$.

(b2) If $\left(M_{k}, \omega_{k}, \psi_{k}\right)$ is a GKM space then $\widehat{\Lambda}_{q_{h}}^{-} \prod_{i=1}^{|\gamma|} \frac{1}{\bar{\psi}_{h}(q)-\bar{\psi}_{h}\left(\gamma_{i}\right)}$ can be written as the product of distinct positive weights in $\Pi_{q}\left(M_{k}\right)$ and a unit in $A_{+}$.

Here, in the case that $M_{k}$ is a GKM space, we use the fact that by Remark 5.7 $M_{j}$ is also a GKM space for all $j$; moreover by Lemma $5.11 \rho_{j}$ is a weight preserving map for all $j$, hence $\pi_{h}$ is weight preserving as well and $\Pi_{q_{h}}\left(X_{h}\right) \subset$ $\Pi_{q_{h}}\left(M_{h}\right)$ is a subset of $\Pi_{q}\left(M_{k}\right)$.

Since $\left[\omega_{h}+\psi_{h}\right]$ is an integral class, Lemma 3.4 and (11) together imply that for all $1 \leq i \leq|\gamma|$,

$$
\begin{aligned}
& \left(\bar{\psi}_{h}\left(\gamma_{i+1}\right)-\bar{\psi}_{h}\left(\gamma_{i}\right)\right) \frac{\alpha_{\gamma_{i}}\left(\gamma_{i+1}\right)}{\Lambda_{\gamma_{i+1}}^{-}} \\
& \quad \in \begin{cases}A, & \text { and } \\
A_{+} & \text {if } \alpha_{r}\left(r^{\prime}\right) \text { is positive } \forall\left(r, r^{\prime}\right) \in E .\end{cases}
\end{aligned}
$$

If $M_{k}$ is a GKM space then by Theorem 2.4, $\frac{\alpha_{r}\left(r^{\prime}\right)}{\Lambda_{r^{\prime}}^{-}}=\frac{\Theta\left(r, r^{\prime}\right)}{\eta\left(r, r^{\prime}\right)}$ for all $\left(r, r^{\prime}\right) \in$ $E$. Moreover Lemma 1.1 implies that $\psi_{k}^{\xi}(r)<\psi_{k}^{\xi}\left(r^{\prime}\right)$ and so $\eta\left(r, r^{\prime}\right)$ is positive because it is a positive multiple of $\psi_{k}\left(r^{\prime}\right)-\psi_{k}(r)$. Therefore, if $M_{k}$ is GKM, then

$$
\alpha_{r}\left(r^{\prime}\right) \text { is positive exactly if } \Theta\left(r, r^{\prime}\right)>0 \text { for all }\left(r, r^{\prime}\right) \in E \text {. }
$$

Moreover, $\frac{\bar{\psi}_{h}\left(\gamma_{i+1}\right)-\bar{\psi}_{h}\left(\gamma_{i}\right)}{\eta\left(\gamma_{i}, \gamma_{i+1}\right)}=m\left(\pi_{h}\left(\gamma_{i}\right), \pi_{h}\left(\gamma_{i+1}\right)\right)$ because $\pi_{h}$ is a weight preserving map. Hence by Lemma 5.18, we have that

$$
\left(\bar{\psi}_{h}\left(\gamma_{i+1}\right)-\bar{\psi}_{h}\left(\gamma_{i}\right)\right) \frac{\alpha_{\gamma_{i}}\left(\gamma_{i+1}\right)}{\Lambda_{\gamma_{i+1}}^{-}} \in A_{+}^{\times} \Leftrightarrow \Theta\left(\gamma_{i}, \gamma_{i+1}\right) \in A_{+}^{\times},
$$

where $A_{+}^{\times}$denotes the set of positive units in $A$. The claim now follows from (a2), (b2), (12), (13) and (14).

Proof of Corollary 5.8. The proof uses the following lemma.

Lemma 5.21. Let $(M, \omega, \psi)$ and $(\widetilde{M}, \widetilde{\omega}, \widetilde{\psi})$ be Hamiltonian T-manifolds with discrete-fixed sets, and let $\pi: M \rightarrow \widetilde{M}$ be a strong symplectic fibration. Let $\varphi=\psi^{\xi}$ be a generic component of the moment map. Given $q \in M^{T}$, 
consider the fiber $\widehat{M}_{q}=\pi^{-1}(\pi(q))$. If $\alpha_{s} \in H_{T}^{2 \lambda(s)}(M ; A)$ is a canonical class at $s \in \widehat{M}_{q}^{T}$, then there exists $\widehat{\alpha}_{s} \in H_{T}^{*}\left(\widehat{M}_{q} ; A\right)$ such that

$$
\widetilde{\Lambda}_{\pi(q)}^{-} \widehat{\alpha}_{s}=\left.\alpha_{s}\right|_{\widehat{M}_{q}} .
$$

Proof. Define $\bar{\varphi}=\pi^{*}(\widetilde{\psi})^{\xi}: M \rightarrow \mathbb{R}$. Since $\widetilde{M}$ has a discrete-fixed set, $\widetilde{\psi}^{\xi}: \widetilde{M} \rightarrow \mathbb{R}$ is a Morse function with critical set $\widetilde{M}^{T}$. Since $\pi$ is a fiber bundle, this implies that $\bar{\varphi}$ is an invariant Morse-Bott function on $M$ and that the critical component of $\bar{\varphi}$ that contains $q$ is the fiber $\widehat{M}_{q}$. Moreover, the index of $\bar{\varphi}$ at $\widehat{M}_{q}$ is $2 \widetilde{\lambda}(\pi(q))$, and the equivariant Euler class of the negative normal bundle of $\bar{\varphi}$ at $\widehat{M}_{q}$ is $\widetilde{\Lambda}_{\pi(q)}^{-}$. By the definition of strong symplectic fibration, Lemma 5.14 and Lemma 5.15 imply that for any $s \in \widehat{M}_{q}^{T}$ the restriction of $\alpha_{s}$ to $\bar{\varphi}^{-1}(-\infty, \bar{\varphi}(q)-\delta)$ vanishes for all $\delta>0$. Thus, by a standard Morse theory argument, there exists $\widehat{\alpha}_{s} \in H_{T}^{2 \lambda(s)-2 \tilde{\lambda}(\pi(q))}\left(\widehat{M}_{q} ; A\right)$ such that $\widetilde{\Lambda}_{\pi(q)}^{-} \widehat{\alpha}_{s}=\left.\alpha_{s}\right|_{\widehat{M}_{q}}$.

Proof of Corollary 5.8. Since $\pi$ is a strong symplectic fibration, $\Lambda_{s}^{-}=$ $\widetilde{\Lambda}_{\pi(s)}^{-} \widehat{\Lambda}_{s}^{-}=\widetilde{\Lambda}_{\pi(q)}^{-} \widehat{\Lambda}_{s}^{-}$and $\lambda(s)=\widetilde{\lambda}(\pi(q))+\widehat{\lambda}(s)$ for all $s \in \widehat{M}_{q}^{T}$. Hence, $\widehat{\lambda}(r) \leq \widehat{\lambda}(s)$ exactly if $\lambda(r) \leq \lambda(s)$ for all $r$ and $s$ in $\widehat{M}_{q}^{T}$.

By Lemma 5.21, for all $s \in \widehat{M}_{q}^{T}$ there exists a class $\widehat{\alpha}_{s} \in H_{T}^{2 \widehat{\lambda}(s)}\left(\widehat{M}_{q} ; A\right)$ such that $\widetilde{\Lambda}_{\pi(q)}^{-} \widehat{\alpha}_{s}=\left.\alpha_{s}\right|_{\widehat{M}_{q}}$. Since $\alpha_{s} \in H_{T}^{2 \lambda(s)}(M ; A)$ is a canonical class, the paragraph above implies that $\widehat{\alpha}_{s}$ is a canonical class at $s$ on $\widehat{M}_{q}$ with respect to the restriction $\left.\varphi\right|_{\widehat{M}_{q}}$. This proves the first claim. Moreover, applying Theorem 0.3 (and Remark 3.1) to $\widehat{M}_{q}$, we have

$$
\widehat{\alpha}_{s}(q)=\widehat{\Lambda}_{q}^{-} \sum_{\gamma \in \widehat{\Sigma}(s, q)} \prod_{i=1}^{|\gamma|} \frac{\psi\left(\gamma_{i+1}\right)-\psi\left(\gamma_{i}\right)}{\psi(q)-\psi\left(\gamma_{i}\right)} \frac{\alpha_{\gamma_{i}}\left(\gamma_{i+1}\right)}{\Lambda_{\gamma_{i+1}}^{-}} \quad \text { for all } s \in \widehat{M}_{q}^{T},
$$

where $\widehat{\Sigma}(s, q)$ is the set of paths from $s$ to $q$ in the canonical graph associated to $\widehat{M}_{q}$.

Now, we can apply Theorem 5.5 to $\pi: M \rightarrow \widetilde{M}$. Observe that a path $\gamma=\left(\gamma_{1}, \ldots, \gamma_{|\gamma|+1}\right)$ from $p$ to $q$ lies in $C(p, q)$ exactly if there exists $j \in$ $\{1, \ldots,|\gamma|+1\}$ such that $\pi\left(\gamma_{i}\right) \neq \pi\left(\gamma_{i+1}\right)$ for all $i<j$ and $\pi\left(\gamma_{i}\right)=\pi\left(\gamma_{i+1}\right)$ for all $i \geq j$, that is, so that $\left(\gamma_{1}, \ldots, \gamma_{j}\right)$ belongs to $\bar{\Sigma}\left(p, \gamma_{j}\right)$, and $\left(\gamma_{j}, \ldots, \gamma_{|\gamma|+1}\right)$ belongs to $\widehat{\Sigma}\left(\gamma_{j}, q\right)$. Hence, since $\Lambda_{q}^{-}=\widehat{\Lambda}_{q}^{-} \widetilde{\Lambda}_{\pi(q)}^{-}$, the second claim follows immediately from (15) and Theorem 5.5.

Finally, the third claim follows from (a1) and (b1). 
Remark 5.22. Claim 1. of Corollary 5.8 is much easier to prove for GKM spaces. To see this, let $(M, \omega, \psi)$ and $(\widetilde{M}, \widetilde{\omega}, \widetilde{\psi})$ be GKM spaces, and let $\pi: M \rightarrow \widetilde{M}$ be a strong symplectic fibration. Let $\varphi=\psi^{\xi}$ be a generic component of the moment map. Assume that canonical classes $\alpha_{p} \in H_{T}^{2 \lambda(p)}(M ; A)$ exist for all $p \in M^{T}$. By Remark 4.3 in $[\mathbf{G T}]$, this implies that $\varphi$ is index increasing on $M$. Since $\pi$ is a strong symplectic fibration, $\left(\widehat{M}_{q},\left.\omega\right|_{\widehat{M}_{q}},\left.\psi\right|_{\widehat{M}_{q}}\right)$ is a GKM space for all $q \in M^{T}$, and its GKM graph is just the restriction of the GKM graph of $M$ to $\widehat{M}_{q}^{T}$. Moreover, $\lambda(s)-\lambda(r)=\widehat{\lambda}(s)-\widehat{\lambda}(r)$ for all $r, s \in \widehat{M}_{q}^{T}$; so $\left.\varphi\right|_{\widehat{M}_{q}}$ is also index increasing. Therefore, the claim follows from Theorem 2.4.

In our final lemma, we show how to express the polynomials $P(\gamma)$ appearing in Corollary 5.8 in terms of the magnitudes of the edges of the GKM graph associated to the base; see Definition 5.17.

Definition 5.23 Fix a GKM space $(\widetilde{M}, \widetilde{\omega}, \widetilde{\psi})$ with GKM graph $\left(\widetilde{V}, \widetilde{E}_{\mathrm{GKM}}\right)$, and let $\widetilde{\psi}^{\xi}$ be a generic component of the moment map. Given an ascending path $\widetilde{\gamma}=\left(\widetilde{\gamma}_{1}, \ldots, \widetilde{\gamma}_{|\gamma|+1}\right)$, the set of skipped vertices of $\widetilde{\gamma}$ is defined to be

$$
S V(\widetilde{\gamma})=\left\{r \in \widetilde{V} \mid \widetilde{\psi}^{\xi}(r)<\widetilde{\psi}^{\xi}\left(\widetilde{\gamma}_{|\widetilde{\gamma}|+1}\right)\right\} \backslash\left\{\widetilde{\gamma}_{1}, \ldots, \widetilde{\gamma}_{\mid} \mid+1\right\} .
$$

Lemma 5.24. Let $(M, \omega, \psi)$ and $(\widetilde{M}, \widetilde{\omega}, \widetilde{\psi})$ be GKM spaces with GKM graphs $\left(V, E_{\mathrm{GKM}}\right)$ and $\left(\widetilde{V}, \widetilde{E}_{\mathrm{GKM}}\right)$ and let $\pi: M \rightarrow \widetilde{M}$ be a strong symplectic fibration. Let $\varphi=\psi^{\xi}$ be a generic component of the moment map. Assume that $\varphi$ is index increasing. Also assume that $H^{*}(\widetilde{M} ; A) \simeq$ $H^{*}\left(\mathbb{C P}^{\frac{1}{2} \operatorname{dim}(\widetilde{M})} ; A\right)$.

Given $p$ and $s \in M^{T}$ and a horizontal path $\gamma=\left(\gamma_{1}, \ldots, \gamma_{|\gamma|+1}\right)$ from $p$ to $s$ in the canonical graph $(V, E)$, define

$$
P(\gamma)=\widetilde{\Lambda}_{\pi(s)}^{-} \prod_{i=1}^{|\gamma|} \frac{\widetilde{\psi}\left(\pi\left(\gamma_{i+1}\right)\right)-\widetilde{\psi}\left(\pi\left(\gamma_{i}\right)\right)}{\widetilde{\psi}(\pi(s))-\widetilde{\psi}\left(\pi\left(\gamma_{i}\right)\right)} \frac{\alpha_{\gamma_{i}}\left(\gamma_{i+1}\right)}{\Lambda_{\gamma_{i+1}}^{-}} .
$$

Then

$$
P(\gamma)=\prod_{i=1}^{|\gamma|} \frac{m\left(\pi\left(\gamma_{i}\right), \pi\left(\gamma_{i+1}\right)\right) \Theta\left(\gamma_{i}, \gamma_{i+1}\right)}{m\left(\pi\left(\gamma_{i}\right), \pi(s)\right)} \prod_{r \in S V(\pi(\gamma))} \eta(r, \pi(s)) .
$$

Proof. Observe that by Theorem 2.4, canonical classes $\alpha_{p}$ exist for all $p \in M^{T}$ and $(V, E) \subset\left(V, E_{\mathrm{GKM}}\right)$. By Lemma $5.11, \pi$ is weight preserving; hence $\eta\left(\gamma_{i}, \gamma_{i+1}\right)=\eta\left(\pi\left(\gamma_{i}\right), \pi\left(\gamma_{i+1}\right)\right)$ for all $i$, and by the definition of magnitude $\widetilde{\psi}\left(\pi\left(\gamma_{i+1}\right)\right)-\widetilde{\psi}\left(\pi\left(\gamma_{i}\right)\right)=\eta\left(\gamma_{i}, \gamma_{i+1}\right) m\left(\pi\left(\gamma_{i}\right), \pi\left(\gamma_{i+1}\right)\right)$. Since $H^{*}(\widetilde{M} ; A) \simeq$ 
$H^{*}\left(\mathbb{C P}^{\frac{1}{2} \operatorname{dim}(\widetilde{M})} ; A\right),\left(\widetilde{V}, \widetilde{E}_{\mathrm{GKM}}\right)$ is a complete graph (see the proof of Lemma $5.18)$, and so $\widetilde{\psi}(\pi(s))-\widetilde{\psi}\left(\pi\left(\gamma_{i}\right)\right)=\eta\left(\pi\left(\gamma_{i}\right), \pi(s)\right) m\left(\pi\left(\gamma_{i}\right), \pi(s)\right)$ for all $i \leq|\gamma|$. Moreover, by Lemma 1.1, $\gamma$ is an ascending path; hence Lemma 5.12 implies that $\pi(\gamma)$ is ascending as well (with respect to $\widetilde{\psi}^{\xi}$ ), and so $\prod_{i=1}^{|\gamma|} \frac{\widetilde{\Lambda}_{\pi(s)}^{-}}{\eta\left(\pi\left(\gamma_{i}\right), \pi(s)\right)}=$ $\prod_{r \in S V(\pi(\gamma))} \eta(r, \pi(s))$. Finally observe that by Theorem $2.4 \frac{\alpha_{\gamma_{i}}\left(\gamma_{i+1}\right)}{\Lambda_{\gamma_{i+1}}^{-}}=$ $\frac{\Theta\left(\gamma_{i}, \gamma_{i+1}\right)}{\eta\left(\gamma_{i}, \gamma_{i+1}\right)}$ for all $i$.

\section{Positive integral formulas for Schubert classes}

We are now ready to apply our results to the important special case of coadjoint orbits. Our main goal is to get positive integral formulas for equivariant Schubert classes on generic coadjoint orbits of type $A_{n}, B_{n}, C_{n}$, and $D_{n}$.

Let $G$ be a compact simple Lie group with Lie algebra $\mathfrak{g}$, and let $(\cdot, \cdot)$ denote the natural pairing between $\mathfrak{g}^{*}$ and $\mathfrak{g}$. Let $T \subset G$ be a maximal torus with Lie algebra $\mathfrak{t}, R \subset \mathfrak{t}^{*}$ denote the set of roots, and $W$ the Weyl group of $G$. Let $\langle\cdot, \cdot\rangle$ be a positive definite symmetric bilinear form on $\mathfrak{g}$ which is $G$-invariant; we use it to embed $\mathfrak{t}^{*}$ in $\mathfrak{g}^{*}$.

Given a point $p_{0} \in \mathfrak{t}^{*}$, consider the coadjoint orbit $\mathcal{O}_{p_{0}}=G \cdot p_{0}$. Let $P_{p_{0}} \subset G$ be the stabilizer of $p_{0}$; the map that takes $g \in G$ to $g \cdot p_{0} \in \mathcal{O}_{p_{0}}$ induces an identification $\mathcal{O}_{p_{0}}=G / P_{p_{0}}$. There is natural $G$-invariant complex structure $J$ and a compatible symplectic form $\omega$ (the Kostant-Kirillov form) on $\mathcal{O}_{p_{0}}$; the moment map is the inclusion map $\mathcal{O}_{p_{0}} \hookrightarrow \mathfrak{g}^{*}$. Hence, the moment map $\psi: \mathcal{O}_{p_{0}} \rightarrow \mathfrak{t}^{*}$ for the $T$ action is the composition of this inclusion with the natural projection from $\mathfrak{g}^{*}$ to $\mathfrak{t}^{*}$. Moreover, $\left(\mathcal{O}_{p_{0}}, \omega, \psi\right)$ is a GKM space. (See $[\mathbf{G H Z}]$.) Finally, we will say that $\mathcal{O}_{p_{0}}$ is generic if $p_{0} \in \mathfrak{t}^{*}$ lies in the interior of a Weyl chamber.

6.1. The canonical graph of a generic coadjoint orbit. Fix a generic coadjoint orbit $\mathcal{O}_{p_{0}}$. As we will see, in this special case, the canonical classes exist and are exactly the equivariant Schubert classes. The main goal of this subsection is to give an explicit description of the associated canonical graph, including all labels.

Proposition 6.1. Let the maximal torus $T$ of a compact simple Lie group $G$ act on a generic coadjoint orbit $\mathcal{O}_{p_{0}} \subset \mathfrak{g}^{*}$ with moment map $\psi: \mathcal{O}_{p_{0}} \rightarrow \mathfrak{t}^{*}$. Let $\varphi=\psi^{\xi}$ be a generic component of the moment map that achieves its minimal value at $p_{0} \in \mathfrak{t}^{*}$, and let $R^{+}=\{\alpha \in R \mid(\alpha, \xi)>0\}$. There exist canonical classes $\alpha_{p} \in H_{T}^{2 \lambda(p)}\left(\mathcal{O}_{p_{0}} ; \mathbb{Z}\right)$ for all $p \in \mathcal{O}_{p_{0}}^{T}$. Under the identification of the Weyl group $W$ with $\mathcal{O}_{p_{0}}^{T}$ given by $w \mapsto w\left(p_{0}\right)$, the canonical graph is $(W, E)$, 
where

$$
\begin{gathered}
\qquad(w)=w\left(p_{0}\right) \quad \text { for all } w \in W, \\
E=\left\{\left(w, w s_{\beta}\right) \in W \times W \mid l\left(w s_{\beta}\right)=l(w)+1 \text { and } \beta \in R\right\}, \quad \text { and } \\
\frac{\alpha_{w\left(p_{0}\right)}\left(w^{\prime}\left(p_{0}\right)\right)}{\Lambda_{w^{\prime}\left(p_{0}\right)}^{-}}=\frac{1}{w(\beta)} \quad \text { for all }\left(w, w^{\prime}\right) \in E \text {, where } w^{\prime}=w s_{\beta} \text { and } \beta \in R^{+} .
\end{gathered}
$$

We begin by describing the GKM graph.

Lemma 6.2. Let the maximal torus $T$ of a compact simple Lie group $G$ act on a generic coadjoint orbit $\mathcal{O}_{p_{0}} \subset \mathfrak{g}^{*}$ with moment map $\psi: \mathcal{O}_{p_{0}} \rightarrow \mathfrak{t}^{*}$. Let $\varphi=\psi^{\xi}$ be a generic component of the moment map that achieves its minimal value at $p_{0} \in \mathfrak{t}^{*}$, and let $R^{+}=\{\alpha \in R \mid(\alpha, \xi)>0\}$. Under the identification of the Weyl group $W$ with $\mathcal{O}_{p_{0}}^{T}$ given by $w \mapsto w\left(p_{0}\right)$, the $G K M$ graph is $\left(W, E_{\mathrm{GKM}}\right)$, where

$$
\begin{gathered}
\psi(w)=w\left(p_{0}\right) \quad \text { for all } w \in W, \\
E_{\mathrm{GKM}}=\left\{\left(w, w s_{\beta}\right) \in W \times W \mid \beta \in R\right\}, \text { and }
\end{gathered}
$$

$\eta\left(w, w^{\prime}\right)=w(\beta)=-w^{\prime}(\beta)$ for all $\left(w, w^{\prime}\right) \in E$, where $w^{\prime}=w s_{\beta}$ and $\beta \in R^{+}$.

Proof. As proved in $[\mathbf{G H Z}]$, the GKM graph $\left(V, E_{\mathrm{GKM}}\right)$ of the coadjoint orbit $\mathcal{O}_{p_{0}}$ can be described as follows:

- The map from the Weyl group $W$ to $\mathfrak{t}^{*}$ which takes $w$ to $w\left(p_{0}\right)$ induces a bijection between the elements of the Weyl group and the vertices $V=\mathcal{O}_{p_{0}}^{T} \subset \mathfrak{t}^{*} \subset \mathfrak{g}^{*}$. The restriction of the moment map $\psi$ to $V$ is the inclusion map, that is, $\psi(p)=p$ for all $p \in V$.

- There exists an edge $e \in E_{\mathrm{GKM}}$ between two vertices $p_{1}=w_{1}\left(p_{0}\right)$ and $p_{2}=w_{2}\left(p_{0}\right)$ if and only if $w_{2}=s_{\alpha} w_{1}$, where $s_{\alpha}$ is the reflection associated to some $\alpha \in R$. In this case, the weight $\eta\left(p_{1}, p_{2}\right)$ is the unique $\alpha \in R$ such that $w_{2}=s_{\alpha} w_{1}$ and $\left\langle p_{2}, \alpha\right\rangle>0$.

In particular, the set of weights of the isotropy representation on $\left(T_{p} \mathcal{O}_{p_{0}}, \omega\right)$ is

$$
\Pi_{p}\left(\mathcal{O}_{p_{0}}\right)=\{\alpha \in R \mid\langle p, \alpha\rangle>0\} \quad \text { for all } p \in V .
$$

Since $p_{0}$ is the minimum, $(\alpha, \xi)<0$ for every weight $\alpha \in \Pi_{p_{0}}\left(\mathcal{O}_{p_{0}}\right)$. By (16), this implies that

$$
\left\langle p_{0}, \alpha\right\rangle<0 \text { for all } \alpha \in R^{+} .
$$

Moreover, it is easy to check that

$$
s_{w(\beta)} w=w s_{\beta} \quad \text { for all } w \in W \text { and } \beta \in R .
$$

Since the Weyl group takes $R$ to itself, this implies that there exists an edge $e \in E_{\mathrm{GKM}}$ between two vertices $p_{1}=w_{1}\left(p_{0}\right)$ and $p_{2}=w_{2}\left(p_{0}\right)$ if and 

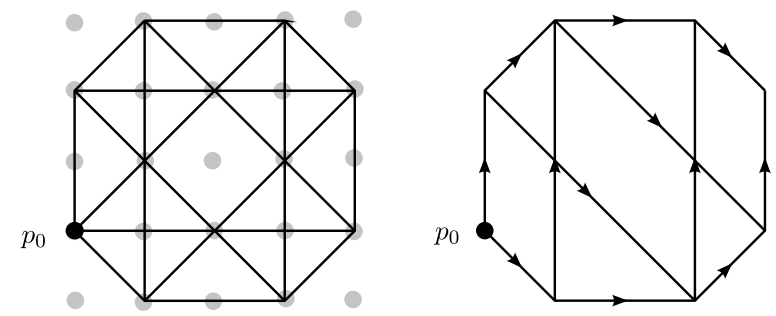

Figure 1. The GKM graph and the canonical graph of a generic coadjoint orbit of $S O(5)$.

only if $w_{2}=w_{1} s_{\beta}$ for some $\beta \in R^{+}$. In this case, since $\langle\cdot, \cdot\rangle$ is $G$-invariant, (17) implies that $\left\langle p_{2}, w_{2}(\beta)\right\rangle=\left\langle w_{2}\left(p_{0}\right), w_{2}(\beta)\right\rangle=\left\langle p_{0}, \beta\right\rangle<0$. Therefore, $\eta\left(p_{1}, p_{2}\right)=-w_{2}(\beta)=-w_{1} s_{\beta}(\beta)=w_{1}(\beta)$.

In Figure 1, we draw the GKM graph of a generic coadjoint orbit of $S O(5)$ through the point $p_{0}=-2 x_{1}-x_{2}$ (here every pair of edges $(p, q)$ and $(q, p)$ is represented by one single edge), together with the canonical graph associated to a component of the moment map, which achieves its minimum at $p_{0}$.

We will need the following standard facts about root systems [Hum]. Given a set of positive roots $R^{+}$, let $R_{0} \subset R^{+}$be the associated simple roots. Every element $w$ of the Weyl group $W$ can be written as a product of simple reflections, i.e., $w=s_{1}, \ldots, s_{r}$, where $s_{i}=s_{\alpha_{i}}$ and $\alpha_{i} \in R_{0}$ for all $i=1, \ldots, r$ [Hum, Section 1.5]. The length of $w$, denoted $l(w)$, is the smallest $r$ for which such an expression exists. Any such expression with $r=l(w)$ is a reduced expression for $w$.

1. Given $w \in W$ and $\beta \in R^{+}, l\left(w s_{\beta}\right)>l(w)$ exactly if $w(\beta) \in R^{+}$[Hum, Section 5.7].

2. If $w=s_{1} \cdots s_{r}$ is a reduced expression for $w \in W$, where $s_{i}=s_{\alpha_{i}}$ for some $\alpha_{i} \in R_{0}$ for all $i$, then (see [Hum, page 14])

$$
R^{+} \cap w^{-1}\left(-R^{+}\right)=\left\{\beta_{1}, \ldots, \beta_{r}\right\} \quad \text { where } \beta_{i}=s_{r} \cdots s_{i+1}\left(\alpha_{i}\right) .
$$

Moreover, the $\beta_{i}$ are distinct.

Lemma 6.3. Let the maximal torus $T$ of a compact simple Lie group $G$ act on a generic coadjoint orbit $\mathcal{O}_{p_{0}} \subset \mathfrak{g}^{*}$ with moment map $\psi: \mathcal{O}_{p_{0}} \rightarrow \mathfrak{t}^{*}$. Let $\varphi=\psi^{\xi}$ be a generic component of the moment map that achieves its minimal value at $p_{0} \in \mathfrak{t}^{*}$, and let $R^{+}=\{\alpha \in R \mid(\alpha, \xi)>0\}$. Then for any $w \in W$ with reduced expression $w=s_{1} \ldots s_{r}$,

$$
\Pi_{w\left(p_{0}\right)}^{-}\left(\mathcal{O}_{p_{0}}\right)=\left\{\eta_{1}, \ldots, \eta_{r}\right\}, \quad \text { where } \eta_{i}=s_{1} \cdots s_{i-1}\left(\alpha_{i}\right) ;
$$

moreover, the $\eta_{i}$ are distinct. Therefore,

$$
\lambda(p)=l(w), \quad \text { for all } p=w\left(p_{0}\right) \in V .
$$


Proof. By Lemma 6.2, $\Pi_{w\left(p_{0}\right)}\left(\mathcal{O}_{p_{0}}\right)=w\left(-R^{+}\right)$. Thus, since the set of weights $\Pi_{p}^{-}\left(\mathcal{O}_{p_{0}}\right)$ in the negative normal bundle at $p$ is the set of positive weights in the representation $\left(T_{p} \mathcal{O}_{p_{0}}, \omega\right)$,

$$
\begin{gathered}
\Pi_{p}^{-}\left(\mathcal{O}_{p_{0}}\right)=R^{+} \cap w\left(-R^{+}\right)=-w\left(R^{+} \cap w^{-1}\left(-R^{+}\right)\right) \\
\text {for all } p=w\left(p_{0}\right) \in V .
\end{gathered}
$$

Hence, the first claim follows directly from Fact 2. above. Since $\lambda(p)=$ $\left|\Pi_{p}^{-}\left(\mathcal{O}_{p_{0}}\right)\right|$, the next claim is immediate.

By Theorem 2.4, the next lemma demonstrates that canonical classes exist on $\mathcal{O}_{p_{0}}$, thus proving the first claim of Proposition 6.1. Recall that $\varphi$ is index increasing exactly if $\lambda(p)<\lambda(q)$ for every $(p, q) \in E_{\text {GKM }}$ such that $\varphi(p)<\varphi(q)$.

Lemma 6.4. Let the maximal torus $T$ of a compact simple Lie group $G$ act on a generic coadjoint orbit $\mathcal{O}_{p_{0}} \subset \mathfrak{g}^{*}$ with moment map $\psi: \mathcal{O}_{p_{0}} \rightarrow \mathfrak{t}^{*}$. Then each generic component of the moment map, $\varphi=\psi^{\xi}$, is index increasing.

Proof. Assume that $\varphi$ achieves its minimum value at $p_{0} \in \mathfrak{t}^{*}$, and define $R^{+}=\{\alpha \in R \mid(\alpha, \xi)>0\}$. Consider an edge $\left(p_{1}, p_{2}\right)=\left(w_{1}\left(p_{0}\right), w_{2}\left(p_{0}\right)\right) \in$ $E_{\mathrm{GKM}}$ so that $\varphi\left(p_{2}\right)>\varphi\left(p_{1}\right)$. By Lemma 6.2 , there exists $\beta \in R^{+}$so that $w_{2}=w_{1} s_{\beta}$ and $\eta\left(p_{1}, p_{2}\right)=w_{1}(\beta)$. Since $\psi\left(p_{2}\right)-\psi\left(p_{1}\right)$ is a positive multiple of $\eta\left(p_{1}, p_{2}\right)$, the fact that $\varphi\left(p_{2}\right)>\varphi\left(p_{1}\right)$ implies that $w_{1}(\beta) \in R^{+}$. By Fact 1. above, this implies that $l\left(w_{2}\right)=l\left(w_{1} s_{\beta}\right)>l\left(w_{1}\right)$. Therefore, Lemma 6.3 implies that $\lambda\left(p_{2}\right)>\lambda\left(p_{1}\right)$, as required.

Given a choice of positive roots $R^{+}, \mathcal{O}_{p_{0}}$ can be identified as a $T$-space with the flag variety $G_{\mathbb{C}} / B$, where $G_{\mathbb{C}}$ is the complexification of $G$ and $B$ is the Borel subgroup associated to $R^{+}$. In the Schubert calculus literature, there is a well-know basis for $H_{T}^{*}\left(G_{\mathbb{C}} / B ; \mathbb{Z}\right)$, whose elements are called equivariant Schubert classes.

Define

$$
\Lambda_{w}^{-}=\prod\left\{\eta \in R^{+} \mid w^{-1}(\eta) \in-R^{+}\right\} .
$$

For every $w \in W$ there exists a unique element $K_{w} \in H_{T}^{2 l(w)}\left(G_{\mathbb{C}} / B ; \mathbb{Z}\right)$ satisfying the following conditions:

$(1)^{\prime} K_{w}\left(w^{\prime}\right)=0$ for all $w^{\prime} \in W \backslash\{w\}$ such that $l\left(w^{\prime}\right) \leq l(w)$.

$(2)^{\prime} K_{w}(w)=\Lambda_{w}^{-}$.

Moreover the set $\left\{K_{w}\right\}_{w \in W}$ is a basis for $H_{T}^{*}\left(G_{\mathbb{C}} / B ; \mathbb{Z}\right)$ as a module over $H^{*}(B T ; \mathbb{Z})($ see $[\mathbf{K u}])$.

Proposition 6.5. Let the maximal torus $T$ of a compact simple Lie group $G$ act on a generic coadjoint orbit $\mathcal{O}_{p_{0}} \subset \mathfrak{g}^{*}$ with moment map $\psi: \mathcal{O}_{p_{0}} \rightarrow \mathfrak{t}^{*}$. 
Let $\varphi=\psi^{\xi}$ be a generic component of the moment map that achieves its minimal value at $p_{0} \in \mathfrak{t}^{*}$, and let $\left\{\alpha_{p}\right\}_{p \in \mathcal{O}_{p_{0}}^{T}}$ be the canonical classes. Let $G_{\mathbb{C}}$ be the complexification of $G, B$ the Borel subgroup associated to $R^{+}=\{\alpha \in$ $R \mid(\alpha, \xi)>0\}$, and $\left\{K_{w}\right\}_{w \in W}$ be the set of equivariant Schubert classes. The canonical classes are the equivariant Schubert classes, i.e.,

$$
\alpha_{w\left(p_{0}\right)}\left(w^{\prime}\left(p_{0}\right)\right)=K_{w}\left(w^{\prime}\right) \quad \text { for all } w, w^{\prime} \in W .
$$

Proof. Since, by [GT, Lemma 2.7], canonical classes are characterized by properties (1) and (2) in Definition 0.1, it is sufficient to prove that the equivariant Schubert classes also satisfy these properties. But this follows immediately by observing that Lemma 6.2 , Lemma 6.3 , and (20) imply that $(1)$ is equivalent to $\left(1^{\prime}\right)$ and $(2)$ to $\left(2^{\prime}\right)$.

The explicit description of the canonical graph given in Proposition 6.1 follows immediately from Theorem 2.4, Lemmas 6.2 and 6.3, and the following proposition, which describes the integers $\Theta(p, q)$ that appear in Theorem 2.4.

Proposition 6.6. Let the maximal torus $T$ of a compact simple Lie group $G$ act on a generic coadjoint orbit $\mathcal{O}_{p_{0}} \subset \mathfrak{g}^{*}$ with moment map $\psi: \mathcal{O}_{p_{0}} \rightarrow \mathfrak{t}^{*}$. Let $\varphi=\psi^{\xi}$ be a generic component of the moment map. Then

$$
\Theta(p, q)=1 \quad \text { for all }(p, q) \in E_{\mathrm{GKM}} \text { with } \lambda(q)-\lambda(p)=1 .
$$

Proof. Assume that $\varphi$ achieves its minimum value at $p_{0} \in \mathfrak{t}^{*}$, and let $R^{+}=$ $\{\alpha \in R \mid(\alpha, \xi)>0\}$. Consider an edge $(p, q)=\left(w\left(p_{0}\right), w^{\prime}\left(p_{0}\right)\right) \in E_{\mathrm{GKM}}$ such that $\lambda(q)-\lambda(p)=1$. By Lemma 6.2 , there exists $\beta \in R^{+}$so that $w^{\prime}=w s_{\beta}$ and $\eta(p, q)=w(\beta)$. By (18), we can also write $w^{\prime}=s_{\alpha} w$, where $\alpha=w(\beta)=\eta(p, q)$.

Let $\Pi_{p}^{-}\left(\mathcal{O}_{p_{0}}\right)$ and $\Pi_{q}^{-}\left(\mathcal{O}_{p_{0}}\right)$ denote the set of weights in the negative normal bundle of $\varphi$ at $p$ and $q$, respectively. In order to prove that $\Theta(p, q)=1$, it is sufficient to find a bijection $f: \Pi_{p}^{-}\left(\mathcal{O}_{p_{0}}\right) \rightarrow \Pi_{q}^{-}\left(\mathcal{O}_{p_{0}}\right) \backslash\{\alpha\}$ such that for each $\eta \in \Pi_{p}^{-}\left(\mathcal{O}_{p_{0}}\right), \eta=f(\eta) \bmod \alpha$, i.e. there exists an integer $n$ such that $f(\eta)-\eta=n \alpha$.

Let $w^{\prime}=s_{1} s_{2} \cdots s_{r}$ be a reduced expression for $w^{\prime}$, where $s_{i}=s_{\alpha_{i}}$ for some $\alpha_{i} \in R_{0}$ for all $i$. By Lemma $6.3 l\left(w^{\prime}\right)=l(w)+1>l(w)$. Therefore, by the Strong Exchange Condition (see [Hum, Section 5.8]) $w=s_{1} \cdots \widehat{s_{j}} \cdots s_{r}$ for some (unique) $j$, where $\widehat{s_{j}}$ indicates that we are omitting the $j$ 'th term. Let $\widetilde{w}=s_{1} s_{2} \cdots s_{j-1}$. Then by (18) we have that for all $j \leq k \leq r$,

$$
s_{1} s_{2} \cdots s_{k}=\widetilde{w} s_{j} s_{j+1} \cdots s_{k}=s_{\widetilde{w}\left(\alpha_{j}\right)} \widetilde{w} s_{j+1} \cdots s_{k}=s_{\widetilde{w}\left(\alpha_{j}\right)} s_{1} s_{2} \cdots \widehat{s_{j}} \cdots s_{k} .
$$

In particular, $w^{\prime}=s_{\widetilde{w}\left(\alpha_{j}\right)} w$, and so $s_{\widetilde{w}\left(\alpha_{j}\right)}=s_{\alpha}$. Hence,

$$
s_{1} s_{2} \cdots s_{k}\left(\alpha_{k+1}\right) \equiv s_{1} s_{2} \cdots \widehat{s_{j}} \cdots s_{k}\left(\alpha_{k+1}\right) \quad \bmod \alpha \quad \text { for all } j \leq k<r .
$$


Moreover, the fact that $l\left(w^{\prime}\right)>l(w)$ implies that $\alpha>0$. Therefore, Lemma 6.3 implies that $\widetilde{w}\left(\alpha_{j}\right)=\alpha$,

$$
\begin{aligned}
& \Pi_{q}^{-} \backslash\{\alpha\}=\left\{\alpha_{1}, \ldots, s_{1} \cdots s_{j-2}\left(\alpha_{j-1}\right), s_{1} \cdots s_{j}\left(\alpha_{j+1}\right), \ldots, s_{1} \cdots s_{r-1}\left(\alpha_{r}\right)\right\}, \\
& \text { and } \\
& \Pi_{p}^{-}=\left\{\alpha_{1}, \ldots, s_{1} \cdots s_{j-2}\left(\alpha_{j-1}\right), s_{1} \cdots s_{j-1}\left(\alpha_{j+1}\right), \ldots, s_{1} \cdots \widehat{s_{j}} \cdots s_{r-1}\left(\alpha_{r}\right)\right\} .
\end{aligned}
$$

The claim follows immediately.

Remark 6.7. Let $w_{1}$ and $w_{2}$ be two elements of the Weyl group $W$ such that $l\left(w_{1}\right)<l\left(w_{2}\right)$ and $w_{2}=w_{1} s_{\beta}$, for some $\beta \in R$; in this case, we will write $w_{1} \rightarrow w_{2}$. The Bruhat order is the transitive closure of this order, i.e., $w \prec w^{\prime}$ in the Bruhat order if there exists a sequence of elements of the Weyl group $w_{0}, w_{1}, \ldots, w_{m}$ such that $w_{0}=w, w_{m}=w^{\prime}$ and $w_{i} \rightarrow w_{i+1}$ for all $i=0, \ldots, m-1$. By Lemma $6.2,6.3$ and $6.4, w \prec w^{\prime}$ exactly if there exists an ascending path from $w$ to $w^{\prime}$ in $\left(V, E_{\mathrm{GKM}}\right)$.

6.2. Maps between coadjoint orbits. Before turning to consider individual Lie groups, we need to establish a few facts about the maps between different coadjoint orbits.

Consider two points $p_{0}$ and $\widetilde{p}_{0} \in \mathfrak{t}^{*}$ such that $P_{\widetilde{p}_{0}} \supset P_{p_{0}}$, where $P_{p_{0}}$ and $P_{\widetilde{p}_{0}}$ are the stabilizers of $p_{0}$ and $\widetilde{p}_{0}$, respectively. Let $\mathcal{O}_{p_{0}}$ and $\mathcal{O}_{\widetilde{p}_{0}}$ be the coadjoint orbits through $p_{0}$ and $\widetilde{p}_{0}$, respectively, and let $\left(V, E_{\mathrm{GKM}}\right)$ and $\left(\widetilde{V}, \widetilde{E}_{\mathrm{GKM}}\right)$ be the GKM graphs associated to $\mathcal{O}_{p_{0}}$ and $\mathcal{O}_{\widetilde{p}_{0}}$, respectively. Since $\mathcal{O}_{p_{0}}=G / P_{p_{0}}$ and $\mathcal{O}_{\widetilde{p}_{0}} \simeq G / P_{\widetilde{p}_{0}}$, there is a natural projection map

$$
\pi: \quad \begin{array}{cl}
\mathcal{O}_{p_{0}} & \rightarrow \mathcal{O}_{\widetilde{p}_{0}} \\
g \cdot p_{0} & \mapsto g \cdot \widetilde{p}_{0} .
\end{array}
$$

Proposition 6.8. The natural projection $\pi: \mathcal{O}_{p_{0}} \rightarrow \mathcal{O}_{\widetilde{p}_{0}}$ described above is a strong symplectic fibration.

Proof. It is well known that $\pi$ is a $T$-equivariant fiber bundle with symplectic fibers, isomorphic to $P_{\widetilde{p}_{0}} / P_{p_{0}}$. Moreover, we can choose the complex structures $J$ and $\widetilde{J}$ on $\mathcal{O}_{p_{0}}$ and $\mathcal{O}_{\widetilde{p}_{0}}$ so that $\pi$ intertwines them. Hence, the claim is a direct consequence of the discussion in Example $5.2(i)$.

Given any fixed point $q \in \mathcal{O}_{p_{0}}^{T}$, let $\widehat{\mathcal{O}}_{q}=\pi^{-1}(\pi(q))$ be the fiber over $\pi(q)$. It is a GKM space; the associated GKM graph is just the restriction to the fiber of the GKM graph associated to $\mathcal{O}_{p_{0}}$. These fibers are equivariantly symplectomorphic, but only with respect to a non-trivial automorphism of the torus. In [GSZ], the authors analyze projections of GKM spaces from a combinatorial point of view, and describe how the GKM structure of different fibers changes. As our next result shows, this is particularly well behaved when $\mathcal{O}_{p_{0}}$ is a generic coadjoint orbit. 
Proposition 6.9. Assume that $\mathcal{O}_{p_{0}}$ is a generic coadjoint orbit. Let $\varphi=\psi^{\xi}$ be a generic component of the moment map that achieves its minimal value at $p_{0} \in \mathfrak{t}^{*}$. Given any $s \in \mathcal{O}_{p_{0}}^{T}$, let $\widehat{\alpha}_{s} \in H_{T}^{2 \widehat{\lambda}(s)}\left(\widehat{\mathcal{O}}_{s} ; \mathbb{Z}\right)$ be the canonical class $^{5}$ at $s$ with respect to $\left.\varphi\right|_{\widehat{\mathcal{O}}_{s}}$, regarded as a map from $\widehat{\mathcal{O}}_{s}^{T}$ to $H^{*}(B T ; \mathbb{Z})$. Let $\tau \in W$ be an element of the Weyl group such that $\tau\left(p_{0}\right)$ is the point in $\widehat{\mathcal{O}}_{\tau\left(p_{0}\right)}$ at which $\left.\varphi\right|_{\widehat{\mathcal{O}}_{\tau\left(p_{0}\right)}}$ achieves its minimum value. Then

$$
\widehat{\alpha}_{\tau(r)}(\tau(s))=\tau\left(\widehat{\alpha}_{r}(s)\right) \quad \text { for all } r, s \in \widehat{\mathcal{O}}_{p_{0}}^{T} .
$$

Here, by a slight abuse of notation, $\tau: H^{*}(B T ; \mathbb{Z}) \rightarrow H^{*}(B T ; \mathbb{Z})$ is the map induced by $\tau: \mathfrak{t}^{*} \rightarrow \mathfrak{t}^{*}$ under the identification $H^{*}(B T ; \mathbb{R}) \simeq \operatorname{Sym}\left(\mathfrak{t}^{*}\right)$.

Proof. To begin, consider any element $g$ in $N(T)$, the normalizer of $T$. With respect to the automorphism of $T$ given by $t \mapsto g t g^{-1}$, the maps $f: \mathcal{O}_{p_{0}} \rightarrow$ $\mathcal{O}_{p_{0}}$ and $\tilde{f}: \mathcal{O}_{\widetilde{p}_{0}} \rightarrow \mathcal{O}_{\widetilde{p}_{0}}$ given by left multiplication by $g$ are equivariant symplectomorphisms; moreover, $\tilde{f} \circ \pi=\pi \circ f$. Hence, $f$ induces an equivariant symplectomorphism from $\widehat{\mathcal{O}}_{p_{0}}$ to $\widehat{\mathcal{O}}_{g \cdot p_{0}}$.

So assume that $g$ represents $\tau \in W=N(T) / T$, that is, $\tau=\operatorname{Ad}_{g}^{*}: \mathfrak{t}^{*} \rightarrow$ $\mathfrak{t}^{*}$. Since $\operatorname{Ad}_{g^{-1}}^{*}$ is the transpose of $\operatorname{Ad}_{g}$, the homomorphism $t \mapsto g t g^{-1}$ and the linear transformation $\operatorname{Ad}_{g^{-1}}^{*}=\tau^{-1}$ induce the same automorphism of $H^{*}(B T ; \mathbb{Z}) \subset \operatorname{Sym}\left(\mathfrak{t}^{*}\right)$. Hence, if we fix any $r \in \mathcal{O}_{p_{0}}^{T}$, the map $\widehat{\mathcal{O}}_{p_{0}}^{T} \rightarrow$ $H^{*}(B T ; \mathbb{Z})$ defined by $s \mapsto \tau^{-1}\left(\widehat{\alpha}_{\tau(r)}(\tau(s))\right)$ is an equivariant cohomology class on $\widehat{\mathcal{O}}_{p_{0}}$. In fact, this class is the canonical class on $\widehat{\mathcal{O}}_{p_{0}}$ at $r$ with respect to the restriction of $\psi^{\xi^{\prime}}$, where $\xi^{\prime}=A d_{g^{-1}}(\xi)$.

Finally, since $\mathcal{O}_{p_{0}}$ is a generic coadjoint orbit, the set of weights $\Pi_{s}\left(\mathcal{O}_{p_{0}}\right)$ of the isotropy representation on the tangent space at the fixed point $s \in \mathcal{O}_{p_{0}}$ agrees with $\Pi_{p_{0}}\left(\mathcal{O}_{p_{0}}\right)$ up to sign, that is, $\Pi_{s}\left(\mathcal{O}_{p_{0}}\right) \cup-\Pi_{s}\left(\mathcal{O}_{p_{0}}\right)=\Pi_{p_{0}}\left(\mathcal{O}_{p_{0}}\right) \cup$ $-\Pi_{p_{0}}\left(\mathcal{O}_{p_{0}}\right)$. Since $\pi$ is a strong symplectic fibration, this implies that - up to sign $-\Pi_{s}\left(\widehat{\mathcal{O}}_{p_{0}}\right)$ agrees with $\Pi_{p_{0}}\left(\widehat{\mathcal{O}}_{p_{0}}\right)$ for all fixed points $s \in \widehat{\mathcal{O}}_{p_{0}}$. Because $p_{0}$ is the point in $\widehat{\mathcal{O}}_{p_{0}}$ where both $\psi^{\xi}$ and $\psi^{\xi^{\prime}}$ achieve their minimum value, this implies that $(\alpha, \xi)>0$ exactly if $\left(\alpha, \xi^{\prime}\right)>0$ for every weight $\alpha \in \Pi_{s}\left(\widehat{\mathcal{O}}_{p_{0}}\right)$ and each fixed point $s \in \widehat{\mathcal{O}}_{p_{0}}$. Hence, by [GT, Remark 2.4] the canonical classes on $\widehat{\mathcal{O}}_{p_{0}}$ with respect to the restriction of $\psi^{\xi^{\prime}}$ are exactly the canonical classes with respect to the restriction of $\psi^{\xi}$.

Finally, given the projection $\pi: \mathcal{O}_{p_{0}} \rightarrow \mathcal{O}_{\widetilde{p}_{0}}$, we can describe explicitly how to lift ascending paths in $\left(\widetilde{V}, \widetilde{E}_{\mathrm{GKM}}\right)$.

\footnotetext{
${ }^{5}$ These classes exist by Corollary 5.8 and Proposition 6.8. Here, $2 \widehat{\lambda}(s)$ is the index of $\left.\varphi\right|_{\widehat{\mathcal{O}}_{s}}$ at $s$
} 
Lemma 6.10. Let $\widetilde{\varphi}: \mathcal{O}_{\widetilde{p}_{0}} \rightarrow \mathbb{R}$ be a generic component of the moment map. Given $p \in V$ and an ascending path $\widetilde{\gamma}$ in $\left(\widetilde{V}, \widetilde{E}_{\mathrm{GKM}}\right)$ that begins at $\pi(p)$, there exists a unique path $\gamma$ of length $|\widetilde{\gamma}|$ in $\left(V, E_{\mathrm{GKM}}\right)$ such that

- $\gamma$ begins at $p$,

- $V(\pi(\gamma))=V(\widetilde{\gamma})$, and

- $\lambda\left(\gamma_{i+1}\right)>\lambda\left(\gamma_{i}\right)$ for all $i$.

If $\widetilde{\gamma}_{i+1}=s_{\beta_{i}}\left(\widetilde{\gamma}_{i}\right)$ for $\beta_{i} \in R$ for each $1 \leq i \leq|\widetilde{\gamma}|$, then the endpoint of $\gamma$ is $w(p)$, where

$$
w=s_{\beta_{|\widetilde{\gamma}|}} s_{\beta_{|\widetilde{\gamma}|-1}} \ldots s_{\beta_{1}} .
$$

Proof. Fix $p \in V$. Since $\pi$ is an equivariant fiber bundle, there is a unique lift $\gamma$ of each path $\widetilde{\gamma}$ starting at $p$, that is, a unique path $\gamma$ of length $|\widetilde{\gamma}|$ in $\left(V, E_{\mathrm{GKM}}\right)$ that starts at $p$ such that $\pi\left(\gamma_{i}, \gamma_{i+1}\right)=\left(\widetilde{\gamma}_{i}, \widetilde{\gamma}_{i+1}\right)$ for all $i$. By Lemma 5.12 and Lemma $6.4, \lambda\left(\gamma_{i+1}\right)>\lambda\left(\gamma_{i}\right)$ for all $i$ exactly if $\widetilde{\gamma}$ is ascending; this proves the first claim. The second claim is an consequence of the fact that $\pi: \mathcal{O}_{p_{0}} \rightarrow \mathcal{O}_{\widetilde{p}_{0}}$ satisfies $\pi\left(w\left(p_{0}\right)\right)=w\left(\pi\left(p_{0}\right)\right)$ for all $w \in W$.

6.3. Generic coadjoint orbits of type $\boldsymbol{A}_{\boldsymbol{n}}$. As we will see below, a generic coadjoint orbit of $S U(n)$ is a tower of complex projective spaces over $\mathbb{Z}$. Therefore, Theorem 5.5 (together with the results of Section 6.1) immediately implies that in this case each restriction of any equivariant Schubert class can be expressed as a sum of terms $\Xi(\gamma)$ over certain paths $\gamma$, where each term is the product of distinct positive roots. In Proposition 6.11 below, we give this description explicitly; this formula is equivalent to a particular case of the combinatorial formula given in [AJS] (Appendix D.3) and [B], as proved by Zara in $[\mathbf{Z a}]$.

Let $G=S U(n+1)$, and let $T \subset G$ be the subtorus of diagonal matrices. Under the natural identification of the dual of the Lie algebra of $T$ as $\mathbf{t}^{*}=$ $\left\{\mu \in\left(\mathbb{R}^{n+1}\right)^{*} \mid \sum_{i=1}^{n+1} \mu_{i}=0\right\}$, the roots are the vectors $x_{i}-x_{j} \in \mathfrak{t}^{*}$ for all $1 \leq i \neq j \leq n$. Here, and throughout this section, $\left\{x_{i}\right\}_{i=1}^{n+1}$ is the standard basis of $\left(\mathbb{R}^{n+1}\right)^{*}$. The Weyl group $\mathcal{S}_{n+1}$ of $G$ is the group of permutations of $n+1$ elements.

Proposition 6.11. Let $B \subset G_{\mathbb{C}}$ be the Borel subgroup associated to the positive roots $R^{+}=\left\{x_{i}-x_{j} \mid 1 \leq i<j \leq n+1\right\}$, where $G_{\mathbb{C}}$ is the complexification of $G=S U(n+1)$. Given $w$ and $w^{\prime}$ in $\mathcal{S}_{n+1}$, let $K_{w} \in H_{T}^{2 l(w)}\left(G_{\mathbb{C}} / B ; \mathbb{Z}\right)$ be the equivariant Schubert class associated to $w$. Let $C\left(w, w^{\prime}\right)$ be the set of tuples $\underline{\sigma}=\left(\sigma_{1}, \ldots, \sigma_{|\underline{\sigma}|+1}\right) \in\left(\mathcal{S}_{n+1}\right)^{|\underline{\sigma}|+1}$ such that $\sigma_{1}=w, \sigma_{|\underline{\sigma}|+1}=w^{\prime}$, and the following properties hold for all $1 \leq i \leq|\underline{\sigma}|$ :

- $l\left(\sigma_{i+1}\right)=l\left(\sigma_{i}\right)+1$;

- $\sigma_{i+1}=\sigma_{i} s_{x_{h_{i}}-x_{k_{i}}}$ for some $1 \leq h_{i}<k_{i} \leq n+1$; and

- $h_{i} \leq h_{i+1}$. 
(1) For all $w$ and $w^{\prime}$ in $\mathcal{S}_{n+1}$,

$$
\begin{aligned}
K_{w}\left(w^{\prime}\right) & =\sum_{\underline{\sigma} \in C\left(w, w^{\prime}\right)} \Xi(\underline{\sigma}), \text { where } \\
\Xi(\underline{\sigma}) & =\Lambda_{w^{\prime}}^{-}\left(\prod_{i=1}^{|\underline{\sigma}|} \frac{1}{x_{\sigma_{i}\left(h_{i}\right)}-x_{\sigma_{\mid \underline{\underline{\sigma}}+1}\left(h_{i}\right)}}\right) \quad \text { for all } \underline{\sigma} \in C\left(w, w^{\prime}\right) .
\end{aligned}
$$

(2) For all $\underline{\sigma} \in C\left(w, w^{\prime}\right), \Xi(\underline{\sigma})$ is the product of distinct positive roots.

Proof. For each $0 \leq j \leq n$, fix a point

$$
\mu^{j} \in \mathfrak{t}^{*} \quad \text { such that } \mu_{1}^{j}<\cdots<\mu_{j}^{j}<\mu_{j+1}^{j}=\cdots=\mu_{n+1}^{j} ;
$$

for simplicity assume that $\mu_{j+1}^{j}=\mu_{j}^{j}+1$. Let $\left(\mathcal{O}_{\mu^{j}}, \omega_{j}, \psi_{j}\right)$ be the coadjoint orbit through $\mu^{j}$ for each $j$. Observe that $\mathcal{O}_{\mu^{0}}$ is a single point and that $\mathcal{O}_{\mu^{n}}$ is isomorphic to $\mathcal{F l}\left(\mathbb{C}^{n+1}\right)$, the variety of complete flags in $\mathbb{C}^{n+1}$. The stabilizer of $\mu^{j}$ is

$$
P_{\mu^{j}}=S(U(1) \times \cdots \times U(1) \times U(n-j+1)) \quad \text { for all } j ;
$$

in particular, $P_{\mu^{j+1}} \subset P_{\mu^{j}}$. By Proposition 6.8, the natural projection map $\rho_{j}: \mathcal{O}_{\mu^{j+1}} \rightarrow \mathcal{O}_{\mu^{j}}$ is a strong symplectic fibration with fiber $P_{\mu^{j}} / P_{\mu^{j+1}} \simeq$ $\mathbb{C} P^{n-j}$ for all $0 \leq j<n$. So $\mathcal{O}_{\mu^{n}}$ is a tower of complex projective spaces over $\mathbb{Z}$.

Each element $\sigma \in \mathcal{S}_{n+1}$ can be represented in one line notation by $\sigma=$ $\sigma(1), \ldots, \sigma(n+1)$; the action of $\sigma$ on a point $\mu=\sum_{i=1}^{n+1} \mu_{i} x_{i} \in \mathfrak{t}^{*}$ is given by $\sigma(\mu)=\sum_{i=1}^{n+1} \mu_{i} x_{\sigma(i)}$. Let $\pi_{j}=\rho_{j} \circ \rho_{j+1} \circ \cdots \circ \rho_{n-1}: \mathcal{O}_{\mu^{n}} \rightarrow \mathcal{O}_{\mu^{j}}$, and define

$$
h\left(\sigma, \sigma^{\prime}\right)=\min \left\{j \in\{1, \ldots, n\} \mid \pi_{j}\left(\sigma\left(\mu^{n}\right)\right) \neq \pi_{j}\left(\sigma^{\prime}\left(\mu^{n}\right)\right)\right\} \forall \sigma \neq \sigma^{\prime} \text { in } \mathcal{S}_{n+1} .
$$

Fix any distinct $\sigma$ and $\sigma^{\prime}$ in $\mathcal{S}_{n+1}$. Since $\pi_{j}\left(\sigma\left(\mu^{n}\right)\right)=\sigma\left(\mu^{j}\right)$ and $\mu_{i}^{j}=\mu_{j+1}^{j}$ exactly if $i>j, \pi_{j}\left(\sigma\left(\mu^{n}\right)\right)=\pi_{j}\left(\sigma^{\prime}\left(\mu^{n}\right)\right)$ exactly if $\sigma(i)=\sigma^{\prime}(i)$ for all $0 \leq$ $i \leq j$. Hence,

$$
\begin{gathered}
h\left(\sigma, \sigma^{\prime}\right)=\min \left\{j \in\{1, \ldots, n\} \mid \sigma(j) \neq \sigma^{\prime}(j)\right\} ; \quad \text { in particular } \\
h\left(\sigma, \sigma s_{x_{h}-x_{k}}\right)=h \quad \text { for all } 1 \leq h<k \leq n+1 .
\end{gathered}
$$

Let $\bar{\psi}_{j}=\pi^{*}\left(\psi_{j}\right): \mathcal{O}_{\mu^{n}} \rightarrow \mathfrak{t}^{*}$ for all $j$. Since $\psi_{j}: \mathcal{O}_{\mu^{j}}^{T} \rightarrow \mathfrak{t}^{*}$ is the inclusion map, $\bar{\psi}_{j}\left(\sigma\left(\mu^{n}\right)\right)=\sum_{i=1}^{n+1} \mu_{i}^{j} x_{\sigma(i)}$ for all $j$. Since $\sum_{m=1}^{n+1}\left(x_{\sigma^{\prime}(m)}-x_{\sigma(m)}\right)=0$ 
and $\mu_{j}^{j}+1=\mu_{j+1}^{j}=\cdots=\mu_{n+1}^{j}$, this implies that for all $j$

$$
\begin{aligned}
\bar{\psi}_{j}\left(\sigma^{\prime}\left(\mu^{n}\right)\right)-\bar{\psi}_{j}\left(\sigma\left(\mu^{n}\right)\right) & =\sum_{m=1}^{n+1} \mu_{m}^{j}\left(x_{\sigma^{\prime}(m)}-x_{\sigma(m)}\right) \\
& =\sum_{m=1}^{n+1}\left(\mu_{m}^{j}-\mu_{j+1}^{j}\right)\left(x_{\sigma^{\prime}(m)}-x_{\sigma(m)}\right) \\
& =\sum_{m=1}^{j}\left(\mu_{m}^{j}-\mu_{j+1}^{j}\right)\left(x_{\sigma^{\prime}(m)}-x_{\sigma(m)}\right) ; \text { therefore, } \\
\bar{\psi}_{j}\left(\sigma^{\prime}\left(\mu^{n}\right)\right)-\bar{\psi}_{j}\left(\sigma\left(\mu^{n}\right)\right) & =x_{\sigma(j)}-x_{\sigma^{\prime}(j)} \quad \text { for all } j \leq h\left(\sigma, \sigma^{\prime}\right) .
\end{aligned}
$$

Thus, for all $1 \leq h<k \leq n+1$ we have

$$
\bar{\psi}_{h}\left(\sigma s_{x_{h}-x_{k}}\left(\mu^{n}\right)\right)-\bar{\psi}_{h}\left(\sigma\left(\mu^{n}\right)\right)=x_{\sigma(h)}-x_{\sigma(k)}=\sigma\left(x_{h}-x_{k}\right) .
$$

To conclude, let $\varphi=\psi_{n}^{\xi}: \mathcal{O}_{\mu^{n}} \rightarrow \mathbb{R}$ be a generic component of the moment map that achieves its minimum value at $\mu^{n}$. By the definition of $\mu^{n}$, the set $R^{+}$coincides with $\{\alpha \in R \mid(\alpha, \xi)>0\}$. So by Proposition 6.5 , canonical classes on $\mathcal{O}_{\mu^{n}}$ correspond to equivariant Schubert classes on $G_{\mathbb{C}} / B$ through the usual identification of $\mathcal{O}_{\mu_{n}}^{T}$ with $W$. Both claims now follow directly from Theorem 5.5 and Proposition 6.1. (Here, we also use the fact that $h\left(\sigma_{i}, \sigma_{i+1}\right) \leq h\left(\sigma_{i}, \sigma_{|\underline{\sigma}|+1}\right)$ for any $\left.\underline{\sigma}=\left(\sigma_{1}, \ldots, \sigma_{|\underline{\sigma}|+1}\right) \in C\left(w, w^{\prime}\right).\right)$

6.4. Generic coadjoint orbits of type $\boldsymbol{C}_{\boldsymbol{n}}$. Let $G=S p(n)$ be the symplectic group, i.e. the quaternionic unitary group $U(n ; \mathbb{H})$. As we will see below, a generic coadjoint orbit of $S p(n)$ is a tower of complex projective spaces over $\mathbb{Z}$. Therefore, Theorem 5.5 (together with the results of Section 6.1) immediately implies that in this case each restriction of any equivariant Schubert class can be expressed as a sum of terms $\Xi(\gamma)$ over certain paths $\gamma$, where each term is the product of distinct positive roots. In Proposition 6.12 below, we give this description explicitly, cf. [Za].

Let $T \subset G$ be a maximal torus. We can identify the dual of the Lie algebra of $T$ as $\mathfrak{t}^{*}=\left(\mathbb{R}^{n}\right)^{*}$; the roots are the vectors $\pm x_{i} \pm x_{j} \in \mathfrak{t}^{*}$ and $\pm 2 x_{i} \in \mathfrak{t}^{*}$ for all $1 \leq i \neq j \leq n$. Here, and throughout this section, $\left\{x_{i}\right\}_{i=1}^{n}$ is the standard basis of $\left(\mathbb{R}^{n}\right)^{*}$. The Weyl group $W$ of $G$ is the group of signed permutations of $n$ elements. Each element $\tau \in W$ can be represented in one line notation by $\tau=(-1)^{\epsilon_{1}} \sigma(1), \ldots,(-1)^{\epsilon_{n}} \sigma(n)$, where $\epsilon_{i} \in\{0,1\}$ for all $i$ and $\sigma \in \mathcal{S}_{n}$.

Proposition 6.12. Let $B \subset G_{\mathbb{C}}$ be the Borel subgroup associated to the positive roots $R^{+}=\left\{x_{i} \pm x_{j} \mid 1 \leq i<j \leq n\right\} \cup\left\{2 x_{i} \mid 1 \leq i \leq n\right\}$, where $G_{\mathbb{C}}$ is the complexification of $G=S p(n)$. Given $w$ and $w^{\prime}$ in $W$, let $K_{w} \in H_{T}^{2 l(w)}\left(G_{\mathbb{C}} / B ; \mathbb{Z}\right)$ be the equivariant Schubert class associated to w. Let 
$C\left(w, w^{\prime}\right)$ be the set of tuples $\underline{\tau}=\left(\tau_{1}, \ldots, \tau_{|\tau|+1}\right) \in W^{|\underline{\tau}|+1}$ such that $\tau_{1}=w$, $\tau_{|\underline{\mid}|+1}=w^{\prime}$ and the following properties hold for all $1 \leq i \leq|\underline{\tau}|$ :

- $l\left(\tau_{i+1}\right)=l\left(\tau_{i}\right)+1$;

- either $\tau_{i+1}=\tau_{i} s_{x_{h_{i}} \pm x_{k_{i}}}$ for some $1 \leq h_{i}<k_{i} \leq n$ or $\tau_{i+1}=\tau_{i} s_{2 x_{h_{i}}}$ for some $1 \leq h_{i} \leq n$; and

- $h_{i} \leq h_{i+1}$.

Let $\tau_{i}=(-1)^{\epsilon_{1}^{i}} \sigma_{i}(1), \ldots,(-1)^{\epsilon_{n}^{i}} \sigma_{i}(n)$, where $\sigma_{i} \in \mathcal{S}_{n}$ and $\epsilon_{j}^{i} \in\{0,1\}$ for all $i$ and $j$.

(1) For all $w$ and $w^{\prime}$ in $W$,

$$
K_{w}\left(w^{\prime}\right)=\sum_{\underline{\tau} \in C\left(w, w^{\prime}\right)} \Xi(\underline{\tau}), \quad \text { where }
$$

$\Xi(\underline{\tau})=\Lambda_{w^{\prime}}^{-}\left(\prod_{i=1}^{|\underline{\tau}|} \frac{1}{(-1)^{\frac{\epsilon \underline{\tau}_{i} \mid+1}{h_{i}}} x_{\sigma_{|\underline{\tau}|+1}\left(h_{i}\right)}-(-1)^{\epsilon_{h_{i}}} x_{\sigma_{i}\left(h_{i}\right)}}\right)$ for all $\underline{\tau} \in C\left(w, w^{\prime}\right)$.

(2) For all $\underline{\tau} \in C\left(w, w^{\prime}\right), \Xi(\underline{\tau})$ is the product of distinct positive roots.

Proof. For each $0 \leq j \leq n$, fix a point

$$
\mu^{j} \in \mathfrak{t}^{*} \quad \text { such that } \mu_{1}^{j}<\cdots<\mu_{j}^{j}<0=\mu_{j+1}^{j}=\cdots=\mu_{n}^{j} ;
$$

for simplicity assume that $\mu_{j}^{j}=-1$. Let $\left(\mathcal{O}_{\mu^{j}}, \omega_{j}, \psi_{j}\right)$ be the coadjoint orbit through $\mu^{j}$ for each $j$. Observe that $\mathcal{O}_{\mu^{0}}$ is a single point. The stabilizer of $\mu^{j}$ is

$$
P_{\mu^{j}}=S^{1} \times \cdots \times S^{1} \times U(n-j ; \mathbb{H}) \quad \text { for all } j ;
$$

in particular, $P_{\mu^{j+1}} \subset P_{\mu^{j}}$. By Proposition 6.8, the natural projection map $\rho_{j}: \mathcal{O}_{\mu^{j+1}} \rightarrow \mathcal{O}_{\mu^{j}}$ is a strong symplectic fibration with fiber $P_{\mu^{j}} / P_{\mu^{j+1}} \simeq$ $\mathbb{C P}^{2(n-j)-1}$ for all $0 \leq j<n$. So $\mathcal{O}_{\mu^{n}}$ is a tower of complex projective spaces over $\mathbb{Z}$.

Let $\tau=(-1)^{\epsilon_{1}} \sigma(1), \ldots,(-1)^{\epsilon_{n}} \sigma(n)$, where $\epsilon_{i} \in\{0,1\}$ for all $i$ and $\sigma \in$ $\mathcal{S}_{n}$; the action of $\tau$ on a point $\mu=\sum_{i=1}^{n} \mu_{i} x_{i} \in \mathfrak{t}^{*}$ is given by $\tau(\mu)=$ $\sum_{i=1}^{n}(-1)^{\epsilon_{i}} \mu_{i} x_{\sigma(i)}$. Let $\pi_{j}=\rho_{j} \circ \rho_{j+1} \circ \cdots \circ \rho_{n-1}: \mathcal{O}_{\mu^{n}} \rightarrow \mathcal{O}_{\mu^{j}}$, and define

$$
h\left(\tau, \tau^{\prime}\right)=\min \left\{j \in\{1, \ldots, n\} \mid \pi_{j}\left(\tau\left(\mu^{n}\right)\right) \neq \pi_{j}\left(\tau^{\prime}\left(\mu^{n}\right)\right)\right\} \forall \tau \neq \tau^{\prime} \text { in } W .
$$

Let $\tau=(-1)^{\epsilon_{1}} \sigma(1), \ldots,(-1)^{\epsilon_{n}} \sigma(n)$ and $\tau^{\prime}=(-1)^{\epsilon_{1}^{\prime}} \sigma^{\prime}(1), \ldots,(-1)^{\epsilon_{n}^{\prime}} \sigma^{\prime}(n)$ in $W$ be distinct. Since $\pi_{j}\left(\tau\left(\mu^{n}\right)\right)=\tau\left(\mu^{j}\right)$ and $\mu_{i}^{j}=\mu_{j+1}^{j}=0$ exactly if $i>j$, $\pi_{j}\left(\tau\left(\mu^{n}\right)\right)=\pi_{j}\left(\tau^{\prime}\left(\mu^{n}\right)\right)$ exactly if $\sigma(i)=\sigma^{\prime}(i)$ and $\epsilon_{i}=\epsilon_{i}^{\prime}$ for all $0 \leq i \leq j$. 
Hence,

$$
\begin{aligned}
h\left(\tau, \tau^{\prime}\right) & =\min \left\{j \in\{1, \ldots, n\} \mid \sigma(j) \neq \sigma^{\prime}(j) \text { or } \epsilon_{j} \neq \epsilon_{j}^{\prime}\right\} ; \text { in particular, } \\
h\left(\tau, \tau s_{x_{h} \pm x_{k}}\right) & =h \quad \text { for all } 1 \leq h<k \leq n, \quad \text { and } \\
h\left(\tau, \tau s_{2 x_{h}}\right) & =h \quad \text { for all } 1 \leq h \leq n .
\end{aligned}
$$

Let $\bar{\psi}_{j}=\pi^{*}\left(\psi_{j}\right): \mathcal{O}_{\mu^{n}} \rightarrow \mathfrak{t}^{*}$ for all $j$. Since $\psi_{j}: \mathcal{O}_{\mu^{j}}^{T} \rightarrow \mathfrak{t}^{*}$ is the inclusion $\operatorname{map}, \bar{\psi}_{j}\left(\tau\left(\mu^{n}\right)\right)=\sum_{i=1}^{n} \mu_{i}^{j}(-1)^{\epsilon_{i}} x_{\sigma(i)}$ for all $j$. Hence,

$\bar{\psi}_{j}\left(\tau^{\prime}\left(\mu^{n}\right)\right)-\bar{\psi}_{j}\left(\tau\left(\mu^{n}\right)\right)=\sum_{m=1}^{j} \mu_{m}^{j}\left((-1)^{\epsilon_{m}^{\prime}} x_{\sigma^{\prime}(m)}-(-1)^{\epsilon_{m}} x_{\sigma(m)}\right) \quad$ for all $j$,

and so

$\bar{\psi}_{j}\left(\tau^{\prime}\left(\mu^{n}\right)\right)-\bar{\psi}_{j}\left(\tau\left(\mu^{n}\right)\right)=\left((-1)^{\epsilon_{j}} x_{\sigma(j)}-(-1)^{\epsilon_{j}^{\prime}} x_{\sigma^{\prime}(j)}\right) \quad$ for all $j \leq h\left(\tau, \tau^{\prime}\right) ;$

therefore

$$
\bar{\psi}_{h}\left(\tau s_{x_{h} \pm x_{k}}\left(\mu^{n}\right)\right)-\bar{\psi}_{h}\left(\tau\left(\mu^{n}\right)\right)=(-1)^{\epsilon_{h}} x_{\sigma(h)} \mp(-1)^{\epsilon_{k}} x_{\sigma(k)}=\sigma\left(x_{h} \pm x_{k}\right)
$$

for all $1 \leq h<k \leq n$, and

$$
\bar{\psi}_{h}\left(\tau s_{2 x_{h}}\left(\mu^{n}\right)\right)-\bar{\psi}_{h}\left(\tau\left(\mu^{n}\right)\right)=(-1)^{\epsilon_{h}}\left(2 x_{\sigma(h)}\right)=\sigma\left(2 x_{h}\right) \quad \text { for all } 1 \leq h \leq n .
$$

To conclude, let $\varphi=\psi_{n}^{\xi}: \mathcal{O}_{\mu^{n}} \rightarrow \mathbb{R}$ be a generic component of the moment map that achieves its minimum value at $\mu^{n}$. By the definition of $\mu^{n}$, the set $R^{+}$coincides with $\{\alpha \in \mathbb{R} \mid(\alpha, \xi)>0\}$. So by Proposition 6.5 , canonical classes on $\mathcal{O}_{\mu^{n}}$ correspond to equivariant Schubert classes on $G_{\mathbb{C}} / B$ through the usual identification of $\mathcal{O}_{\mu^{n}}^{T}$ with $W$. The claim now follows directly from Theorem 5.5 and Proposition 6.1. (Here, we use the fact that $h\left(\tau_{i}, \tau_{i+1}\right) \leq$ $h\left(\tau_{i}, \tau_{|\underline{\tau}|+1}\right)$ for any $\underline{\tau}=\left(\tau_{1}, \ldots, \tau_{|\underline{\mid}|+1}\right) \in C\left(w, w^{\prime}\right)$.)

6.5. Generic coadjoint orbits of type $\boldsymbol{B}_{\boldsymbol{n}}$. The main result of this section is an inductive positive integral formula that expresses the restrictions of the equivariant Schubert classes on a generic coadjoint orbit of type $B_{n}$ in terms of products of distinct positive roots with positive integer coefficients, and the restriction of equivariant Schubert classes on a generic coadjoint orbit of type $B_{n-1}$. To find this formula, we will apply Corollary 5.8 to the natural projection from a generic coadjoint orbit of $S O(2 n+1)$ to $G r_{2}^{+}\left(\mathbb{R}^{2 n+1}\right)$, the Grassmannian of oriented 2-planes in $\mathbb{R}^{2 n+1}$.

Let $G=S O(2 n+1), T \subset G$ be a maximal torus, and $W$ be the associated Weyl group; assume $n>1$. We can identify the dual of the Lie algebra of $T$ with $\left(\mathbb{R}^{n}\right)^{*}$ so that the set of roots is

$$
R=\left\{ \pm x_{i} \pm x_{j} \mid 1 \leq i<j \leq n\right\} \cup\left\{ \pm x_{i} \mid 1 \leq i \leq n\right\} \subset \mathfrak{t}^{*} .
$$


Let $\widehat{G}=S O(2 n-1)$. We can identify the dual of the Lie algebra of a maximal torus $\widehat{T}$ of $\widehat{G}$ with the set of $\left(a_{1}, \ldots, a_{n}\right) \in \mathfrak{t}^{*}$ such that $a_{1}=0$. This identifies the roots of $\widehat{G}$ with the set

$$
\widehat{R}=\left\{ \pm x_{i} \pm x_{j} \mid 2 \leq i<j \leq n\right\} \cup\left\{ \pm x_{i} \mid 2 \leq i \leq n\right\} \subset R,
$$

and the Weyl group $\widehat{W}$ of $\widehat{G}$ with the subgroup of $W$ generated by reflections across the roots in $\widehat{R}$; moreover, it induces a map from $H^{*}(B \widehat{T} ; \mathbb{Z})$ to $H^{*}(B T ; \mathbb{Z})$. Equivalently, let $\widetilde{V}=\left\{ \pm x_{1}, \pm x_{2}, \ldots, \pm x_{n}\right\} ; \widehat{W}$ is the kernel of the map $\pi: W \rightarrow \widetilde{V}$ defined by $\pi(w)=w\left(-x_{1}\right)$.

To state our main theorem, we will need several additional definitions. Let

$$
R^{+}=\left\{x_{i} \pm x_{j} \mid 1 \leq i<j \leq n\right\} \cup\left\{x_{i} \mid 1 \leq i \leq n\right\} \subset R
$$

be the set of positive roots. Define

$$
E=\left\{\left(\tau, \tau s_{\beta}\right) \in W \times W \mid l\left(\tau s_{\beta}\right)=l(\tau)+1 \text { and } \beta \in R\right\} .
$$

Given $w$ and $w^{\prime} \in W$, let $\bar{\Sigma}\left(w, w^{\prime}\right)$ denote the set of paths $\gamma=\left(\gamma_{1}, \ldots, \gamma_{|\gamma|+1}\right)$ from $w$ to $w^{\prime}$ in $(W, E)$ such that $\pi\left(\gamma_{i}\right) \neq \pi\left(\gamma_{i+1}\right)$ for all $i$. Equivalently, $\bar{\Sigma}\left(w, w^{\prime}\right)$ is the set of paths from $w$ to $w^{\prime}$ such that each edge is of the form $\left(\tau, \tau s_{\beta}\right)$, where $l\left(\tau s_{\beta}\right)=l(\tau)+1$ and $\beta \in R \backslash \widehat{R}$. Given a sequence $\widetilde{\gamma} \in \widetilde{V}^{k}$, let $V(\widetilde{\gamma})$ be the set of "vertices" of $\widetilde{\gamma}$ :

$$
V(\widetilde{\gamma})=\left\{\widetilde{\gamma}_{1}, \ldots, \widetilde{\gamma}_{k}\right\} \subset \widetilde{V}
$$

We need the following lemma, which we prove on page 222 .

Lemma 6.13. Given any path $\gamma=\left(\gamma_{1}, \gamma_{2}, \ldots, \gamma_{|\gamma|+1}\right) \in \bar{\Sigma}\left(w, w^{\prime}\right)$, the sequence $\tilde{\gamma}=\pi(\gamma)=\left(\pi\left(\gamma_{1}\right), \pi\left(\gamma_{2}\right), \ldots, \pi\left(\gamma_{|\gamma|+1}\right)\right)$ is a subsequence of $\left(-x_{1},-x_{2}, \ldots,-x_{n}, x_{n}, \ldots, x_{2}, x_{1}\right)$.

Definition 6.14 A path $\gamma \in \bar{\Sigma}\left(w, w^{\prime}\right)$ with $\pi(\gamma)=\widetilde{\gamma}$ is incomplete if both the following conditions are satisfied:

(i) $\left\{\pi\left(w^{\prime}\right),-\pi\left(w^{\prime}\right)\right\} \subset V(\widetilde{\gamma})$, and

(ii) $\gamma$ does not contain any edge $e$ of the form $\left(\tau, \tau s_{x_{1}}\right)$, that is, an edge such that $\pi(e)=\left(-x_{j}, x_{j}\right)$ for some $j \in\{1, \ldots, n\}$.

Otherwise $\gamma$ is complete.

Definition 6.15 A path $\gamma \in \bar{\Sigma}\left(w, w^{\prime}\right)$ with $\pi(\gamma)=\widetilde{\gamma}$ is relevant if either it is complete or if it is incomplete and $x_{k(\gamma)+1} \in V(\widetilde{\gamma})$, where ${ }^{6} k(\gamma)=\max \{j \mid$ $\left.\left\{-x_{j}, x_{j}\right\} \subset V(\widetilde{\gamma})\right\}$.

\footnotetext{
${ }^{6}$ Observe that if $\gamma$ is incomplete then condition (i) in the definition above implies that $\left\{j \mid\left\{-x_{j}, x_{j}\right\} \subset V(\widetilde{\gamma})\right\} \neq \emptyset$ and-by Lemma 6.13 - condition (ii) implies that $k(\gamma)<n$.
} 
Finally, given a path $\gamma \in \bar{\Sigma}\left(w, w^{\prime}\right)$, define

$$
P(\gamma)=\widetilde{\Lambda}_{\pi\left(w^{\prime}\right)}^{-}\left(\prod_{i=1}^{|\gamma|} \frac{1}{\pi\left(w^{\prime}\right)-\pi\left(\gamma_{i}\right)} \frac{\pi\left(\gamma_{i+1}\right)-\pi\left(\gamma_{i}\right)}{\eta\left(\gamma_{i}, \gamma_{i+1}\right)}\right),
$$

where $\widetilde{\Lambda}_{\pi\left(w^{\prime}\right)}^{-}$is the product of the $\alpha \in R^{+}$such that $\left\langle\pi\left(w^{\prime}\right), \alpha\right\rangle>0$ and $\pi\left(s_{\alpha}\left(w^{\prime}\right)\right) \neq \pi\left(w^{\prime}\right)$.

The main theorem of this section can be stated as follows.

Theorem 6.16. Let $B \subset G_{\mathbb{C}}$ and $\widehat{B} \subset \widehat{G}_{\mathbb{C}}$ be the Borel subgroups associated to $R^{+}$and $R^{+} \cap \widehat{R}$, respectively, where $G_{\mathbb{C}}$ and $\widehat{G}_{\mathbb{C}}$ are the complexifications of $G=S O(2 n+1)$ and $\widehat{G}=S O(2 n-1)$, and other symbols are defined as above. Given $w$ and $w^{\prime}$ in $W$, let $K_{w} \in H_{T}^{2 l(w)}\left(G_{\mathbb{C}} / B ; \mathbb{Z}\right)$ be the equivariant Schubert class associated to $w$, and let $\tau \in W$ be the shortest element such that $\pi(\tau)=$ $\pi\left(w^{\prime}\right)$. For all $\widehat{w} \in \widehat{W}$, let $\widehat{K}_{\widehat{w}} \in H_{\widehat{T}}^{2 l(\widehat{w})}\left(\widehat{G}_{\mathbb{C}} / \widehat{B} ; \mathbb{Z}\right)$ be the equivariant Schubert class associated to $\widehat{w}$, and let $R(w, \tau \widehat{w}) \subset \bar{\Sigma}(w, \tau \widehat{w})$ denote the set of relevant paths from $w$ to $\tau \widehat{w}$ in $(W, E)$.

(1) For all $w$ and $w^{\prime}$ in $W$

$$
K_{w}\left(w^{\prime}\right)=\sum_{\widehat{w} \in \widehat{W}}\left(\sum_{\gamma \in R(w, \tau \widehat{w})} Q(\gamma)\right) \tau\left(\widehat{K}_{\widehat{w}}\left(\tau^{-1} w^{\prime}\right)\right),
$$

where for every $\gamma \in R(w, \tau \widehat{w})$

$$
Q(\gamma)= \begin{cases}P(\gamma) & \text { if } \gamma \text { is complete } \\ P(\gamma) \frac{2 \pi\left(w^{\prime}\right)}{\pi\left(w^{\prime}\right)+x_{k(\gamma)+1}} & \text { if } \gamma \text { is incomplete. }\end{cases}
$$

(2) $Q(\gamma)$ is the product of distinct positive roots and a constant which is either 1 or 2 for all $\gamma \in R(w, \tau \widehat{w})$.

Example 6.17 Consider the case that $G=S O(5)$. Let $w=s_{2}$ and $w^{\prime}=$ $s_{1} s_{2} s_{1} s_{2}$, where $s_{1}=s_{x_{1}-x_{2}}$ and $s_{2}=s_{x_{2}}$. We want to compute $K_{w}\left(w^{\prime}\right)$ using Theorem 6.16. Since $\pi^{-1}\left(\pi\left(w^{\prime}\right)\right)=\left\{\tau, w^{\prime}\right\} \subset W$, where $\tau=s_{1} s_{2} s_{1}, \tau$ is the shortest element in $\pi^{-1}\left(\pi\left(w^{\prime}\right)\right)$. Since $\widehat{W}=\left\{\mathrm{Id}, s_{2}\right\}$ and $\tau s_{2}=w^{\prime}$, we need to find the sets of relevant paths $R(w, \tau)$ and $R\left(w, w^{\prime}\right)$. It is straightforward to check that the following hold:

- $\bar{\Sigma}(w, \tau)=\left\{\gamma^{1}, \gamma^{2}\right\}$, where $\widetilde{\gamma}^{1}=\pi\left(\gamma^{1}\right)=\left(-x_{1}, x_{2}, x_{1}\right)$ and $\widetilde{\gamma}^{2}=$ $\left(-x_{1},-x_{2}, x_{1}\right)$; so the paths $\gamma^{1}$ and $\gamma^{2}$ are incomplete, and $\gamma^{1}$ is relevant. Hence $R(w, \tau)=\left\{\gamma^{1}\right\}$; moreover, $Q\left(\gamma^{1}\right)=x_{1}$.

- $\bar{\Sigma}\left(w, w^{\prime}\right)=\left\{\gamma^{3}\right\}$, where $\widetilde{\gamma}^{3}=\pi\left(\gamma^{3}\right)=\left(-x_{1},-x_{2}, x_{2}, x_{1}\right)$; so the path $\gamma^{3}$ is complete and hence relevant. So $R\left(w, w^{\prime}\right)=\left\{\gamma^{3}\right\}$ and $Q\left(\gamma^{3}\right)=1$. 
Moreover $\widehat{K}_{\text {Id }} \equiv 1, \widehat{K}_{s_{2}}\left(s_{2}\right)=x_{2}$ and $\tau\left(x_{2}\right)=x_{2}$. Therefore, Theorem 6.16 implies that

$$
K_{w}\left(w^{\prime}\right)=Q\left(\gamma^{1}\right) \tau\left(\widehat{K}_{\text {Id }}\left(s_{2}\right)\right)+Q\left(\gamma^{3}\right) \tau\left(\widehat{K}_{s_{2}}\left(s_{2}\right)\right)=x_{1}+x_{2} .
$$

To prove Theorem 6.16, we need to translate it into geometrical language. Fix a point

$\mu^{j} \in \mathfrak{t}^{*}$ such that $\mu_{1}^{j}<\cdots<\mu_{j}^{j}<0=\mu_{j+1}^{j}=\cdots=\mu_{n}^{j}$ for each $0 \leq j \leq n ;$

for simplicity assume that $\mu_{j}^{j}=-1$. Let $\left(\mathcal{O}_{\mu^{j}}, \omega_{j}, \psi_{j}\right)$ be the coadjoint orbit through $\mu^{j}$ for each $j$. The stabilizer of $\mu^{j}$ is

$$
S O(2) \times \cdots \times S O(2) \times S O(2 n-2 j+1) \text { for all } j ;
$$

in particular, $P_{\mu^{i}} \subseteq P_{\mu^{j}}$ for all $1 \leq j \leq i \leq n$. Moreover, let $\varphi=\psi_{n}^{\xi}: \mathcal{O}_{\mu^{n}} \rightarrow$ $\mathbb{R}$ be a generic component of the moment map that achieves its minimum value at $\mu^{n}$. Observe that, by the definition of $\mu^{n}$, the set $R^{+}$coincides with $\{\alpha \in R \mid(\alpha, \xi)>0\}$. By Proposition 6.1, there exists a canonical class $\alpha_{p} \in H_{T}^{2 \lambda(p)}\left(\mathcal{O}_{\mu^{n}} ; \mathbb{Z}\right)$ for each $p \in \mathcal{O}_{\mu^{n}}^{T}$. The map from $W$ to $\mathcal{O}_{\mu^{n}}^{T}$ given by $w \mapsto w\left(\mu^{n}\right)$ identifies the canonical graph of $\mathcal{O}_{\mu^{n}}$ with $(W, E)$; we shall identify these without further comment. Moreover, by Proposition 6.6 and Lemma 6.3, $\Theta\left(r, r^{\prime}\right)=1$ for each edge $\left(r, r^{\prime}\right)$ in $E$.

Let $\pi: \mathcal{O}_{\mu^{n}} \rightarrow \mathcal{O}_{\mu^{1}}$ be the natural projection. Note that $\bar{\Sigma}\left(w, w^{\prime}\right)$ is exactly the set of horizontal paths (with respect to $\pi$ ) from $w$ to $w^{\prime}$; see Definition 5.9. Moreover, given any $\gamma \in \bar{\Sigma}\left(w, w^{\prime}\right)$, the projection $\widetilde{\gamma}=\pi(\gamma)$ is an ascending path in the GKM graph $\left(\widetilde{V}, \widetilde{E}_{\mathrm{GKM}}\right)$ associated to $\left(\mathcal{O}_{\mu^{1}}, \omega_{1}, \psi_{1}\right)$ by Lemmas 5.11 and 5.12 ; this proves Lemma 6.13 . Finally, note that $\left(\widetilde{V}, \widetilde{E}_{\mathrm{GKM}}\right)$ is a complete graph, where $\widetilde{V}=\left\{ \pm x_{1}, \pm x_{2}, \ldots, \pm x_{n}\right\}$, and that $\widetilde{\Lambda}_{\pi\left(w^{\prime}\right)}^{-}$is the equivariant Euler class of the negative normal bundle of $\widetilde{\varphi}=\psi_{1}^{\xi}: \mathcal{O}_{\mu^{1}} \rightarrow \mathbb{R}$ at $\pi\left(w^{\prime}\right)$.

Remark 6.18. By Proposition 6.8, the natural projection map $\rho_{j}: \mathcal{O}_{\mu^{j+1}} \rightarrow$ $\mathcal{O}_{\mu^{j}}$ is a strong symplectic fibration with fiber the Grassmannian $P_{\mu^{j}} / P_{\mu^{j+1}} \simeq G r_{2}^{+}\left(\mathbb{R}^{2 n-2 j+1}\right)$ for all $0 \leq j<n$. Hence, since $H^{*}$ $\left(G r_{2}^{+}\left(\mathbb{R}^{2 n-2 j+1}\right) ; \mathbb{Z}\left[\frac{1}{2}\right]\right) \simeq H^{*}\left(\mathbb{C P}^{2 n-2 j-1} ; \mathbb{Z}\left[\frac{1}{2}\right]\right)$ and $\Theta\left(r, r^{\prime}\right)=1$ for each edge $\left(r, r^{\prime}\right)$ in $E$, Theorem 5.5 (together with Proposition 6.5) immediately implies that, for any $w, w^{\prime} \in W$ we can express the restriction $K_{w}\left(w^{\prime}\right)$ as a sum of terms $\Xi(\gamma)$ over paths $\gamma \in C\left(w, w^{\prime}\right)$ where each term is a polynomial in the simple roots with positive rational coefficients; more precisely, $\Xi(\gamma)$ is the product of distinct positive roots and a constant that is a (possibly negative) power of 2 ; cf. [Za]. 
To prove the theorem, we need to analyze how the expression of $P(\gamma)$ changes depending on whether $\gamma$ is complete or incomplete.

Proposition 6.19. Let $\gamma=\left(\gamma_{1}, \ldots, \gamma_{|\gamma|+1}\right)$ be a path in $\bar{\Sigma}(p, s)$. Let $\widetilde{\gamma}=$ $\pi(\gamma)$ and let $S V(\widetilde{\gamma})$ be the skipped vertices of $\widetilde{\gamma}$; see Definition 5.23. Then

$$
P(\gamma)=c \prod_{r \in S V(\widetilde{\gamma})} \eta(r, \pi(s)), \quad \text { where } c= \begin{cases}1 \text { or } 2 & \text { if } \gamma \text { is complete, and } \\ \frac{1}{2} & \text { if } \gamma \text { is incomplete. }\end{cases}
$$

More precisely, $c=2$ exactly if neither condition in Definition 6.14 is satisfied.

Proof. The edge $\left(-x_{j}, x_{j}\right) \in \widetilde{E}_{\mathrm{GKM}}$ has magnitude 2 for all $j$; all the other edges $\left(r, r^{\prime}\right) \in \widetilde{E}_{\mathrm{GKM}}$ have magnitude 1; see Definition 5.17. Moreover, by Lemma $6.13, \widetilde{\gamma}$ can have at most one edge of type $\left(-x_{j}, x_{j}\right)$. Therefore, since $\Theta\left(r, r^{\prime}\right)=1$ for all $\left(r, r^{\prime}\right) \in E$, the claim follows from Lemma 5.24.

We also need the following two lemmas.

Lemma 6.20. Let $\gamma$ be a path in $\bar{\Sigma}(p, s)$. If $\left\{-x_{l}, x_{l}\right\} \subset V(\pi(\gamma))$ for some $l<n$, then $\left\{-x_{l+1}, x_{l+1}\right\} \cap V(\pi(\gamma)) \neq \emptyset$.

Lemma 6.21. Let $\gamma$ be a path in $\bar{\Sigma}(p, s)$ such that $\left\{-x_{l}, x_{l}\right\} \subset V(\pi(\gamma))$ for some l. If $\left\{-x_{l+1}, x_{l+1}\right\} \cap V(\pi(\gamma))=\left\{x_{l+1}\right\}$ for some $l$, then there exists a unique path $\gamma^{\prime} \in \bar{\Sigma}(p, s)$ such that $V\left(\pi\left(\gamma^{\prime}\right)\right)$ is obtained from $V(\pi(\gamma))$ by replacing the vertex $x_{l+1}$ with $-x_{l+1}$. That is, $-x_{l+1} \in V\left(\pi\left(\gamma^{\prime}\right)\right)$ and $V\left(\pi\left(\gamma^{\prime}\right)\right) \backslash\left\{-x_{l+1}\right\}=V(\pi(\gamma)) \backslash\left\{x_{l+1}\right\}$. A similar claim holds if $\left\{-x_{l+1}, x_{l+1}\right\} \cap V(\pi(\gamma))=\left\{-x_{l+1}\right\}$.

To simplify the proof of these lemmas, let $s_{l}=s_{x_{l}-x_{l+1}}$ denote the reflection across the root $x_{l}-x_{l+1}$ for all $l \in\{1, \ldots, n-1\}$,

We recall the following relations; for all $l \in\{1, \ldots, n-1\}$ and $j \in$ $\{1,2, \ldots, n\}$ with $j \notin\{l, l+1\}$

$$
\begin{aligned}
s_{x_{l}} & =s_{l} s_{x_{l+1}} s_{l}, \\
s_{x_{l} \pm x_{j}} & =s_{l} s_{x_{l+1} \pm x_{j}} s_{l}, \\
s_{x_{l}+x_{l+1}} & =s_{l} s_{x_{l}+x_{l+1}} s_{l} .
\end{aligned}
$$

Proof of Lemma 6.20. Let $\widetilde{\gamma}=\pi(\gamma)$. Suppose that, on the contrary, $\left\{-x_{l}, x_{l}\right\} \subset V(\widetilde{\gamma})$ but $\left\{-x_{l+1}, x_{l+1}\right\} \cap V(\widetilde{\gamma})=\emptyset$. Let $\widetilde{\gamma}^{\prime}$ be the ascending path in $\left(\widetilde{V}, \widetilde{E}_{\mathrm{GKM}}\right)$ such that $V\left(\widetilde{\gamma}^{\prime}\right)=\left\{-x_{l+1}, x_{l+1}\right\} \cup V(\widetilde{\gamma})$. There exists $\beta_{i} \in R$ such that $\widetilde{\gamma}_{i+1}=s_{\beta_{i}}\left(\widetilde{\gamma}_{i}\right)$ for all $i=1, \ldots,|\widetilde{\gamma}|$, and there exists $\delta_{i} \in R$ such that $\widetilde{\gamma}_{i+1}^{\prime}=s_{\delta_{i}}\left(\widetilde{\gamma}_{i}^{\prime}\right)$ for all $i=1, \ldots,\left|\widetilde{\gamma}^{\prime}\right|$. Define $w$ and $w^{\prime}$ in the Weyl group $W$ of $G$ by

$$
w=s_{\beta_{|\widetilde{\gamma}|}} s_{\beta_{|\widetilde{\gamma}|-1}} \cdots s_{\beta_{1}} \text { and } \quad w^{\prime}=s_{\delta_{\left|\tilde{\gamma}^{\prime}\right|}} s_{\delta_{\left|\tilde{\gamma}^{\prime}\right|-1}} \cdots s_{\delta_{1}} .
$$


- If $\widetilde{\gamma}=\left(\ldots,-x_{l}, x_{l}, \ldots\right)$, then $w=w_{1} s_{x_{l}} w_{2}$ and $w^{\prime}=w_{1} s_{l} s_{x_{l+1}} s_{l} w_{2}$ for some $w_{1}$ and $w_{2} \in W$. Hence $w=w^{\prime}$ by (22).

- If $\left(-x_{l}, x_{l}\right)$ is not an edge of $\widetilde{\gamma}$, then there exists $i$ and $h>l$ so that $w=w_{1} s_{x_{l} \pm x_{h}} w_{0} s_{x_{l} \pm x_{i}} w_{2}$ and $w^{\prime}=w_{1} s_{l} s_{x_{l+1} \pm x_{h}} w_{0} s_{x_{l+1} \pm x_{i}} s_{l} w_{2}$ for some $w_{0}, w_{1}$, and $w_{2} \in W$ such that $w_{0}$ commutes with $s_{l}$. Hence by (22) we again have $w^{\prime}=w_{1} s_{l} s_{x_{l+1} \pm x_{h}} s_{l} w_{0} s_{l} s_{x_{l+1} \pm x_{i}} s_{l} w_{2}=$ $w_{1} s_{x_{l} \pm x_{h}} w_{0} s_{x_{l} \pm x_{i}} w_{2}=w$.

Moreover, by Lemma 6.10 there exists a path $\gamma^{\prime}$ of length $\left|\widetilde{\gamma}^{\prime}\right|$ in $\left(V, E_{\mathrm{GKM}}\right)$ that starts at $p$ such that $V\left(\pi\left(\gamma^{\prime}\right)\right)=V\left(\widetilde{\gamma}^{\prime}\right)$ and $\lambda\left(\gamma_{i+1}^{\prime}\right)>\lambda\left(\gamma_{i}^{\prime}\right)$ for all $1 \leq$ $i \leq\left|\widetilde{\gamma}^{\prime}\right|$. Moreover, since $w=w^{\prime}$, the endpoints $s=w(p)$ of $\gamma$ and $s^{\prime}=w^{\prime}(p)$ of $\gamma^{\prime}$ are equal. On the other hand, the fact that $\gamma \in \bar{\Sigma}(p, s) \subset \Sigma(p, s)$ implies that $\lambda(s)-\lambda(p)=|\widetilde{\gamma}|$. Moreover, $\lambda\left(\gamma_{i+1}^{\prime}\right)>\lambda\left(\gamma_{i}^{\prime}\right)$ for all $1 \leq i \leq\left|\widetilde{\gamma}^{\prime}\right|$. Hence, $\lambda\left(s^{\prime}\right)-\lambda(p) \geq\left|\widetilde{\gamma}^{\prime}\right|=|\widetilde{\gamma}|+2$. Since $s=s^{\prime}$, this is impossible.

Proof of Lemma 6.21. Let $\widetilde{\gamma}=\pi(\gamma)$. Assume that $\left\{-x_{l+1}, x_{l+1}\right\} \cap V(\widetilde{\gamma})=$ $\left\{x_{l+1}\right\}$. Let $\widetilde{\gamma}^{\prime}$ be the ascending path in $\left(\widetilde{V}, \widetilde{E}_{\mathrm{GKM}}\right)$ such that $V\left(\widetilde{\gamma}^{\prime}\right)$ is obtained from $V(\widetilde{\gamma})$ by replacing the vertex $x_{l+1}$ with $-x_{l+1}$. As before, there exists $\beta_{i} \in R$ such that $\gamma_{i+1}=s_{\beta_{i}}\left(\gamma_{i}\right)$ for all $i=1, \ldots,|\gamma|$, and there exists $\delta_{i} \in R$ such that $\gamma_{i+1}^{\prime}=s_{\delta_{i}}\left(\gamma_{i}^{\prime}\right)$ for all $i=1, \ldots,\left|\gamma^{\prime}\right|$. By Lemma 6.10, this implies that $\widetilde{\gamma}_{i+1}=s_{\beta_{i}}\left(\widetilde{\gamma}_{i}\right)$ and $\widetilde{\gamma}_{i+1}^{\prime}=s_{\delta_{i}}\left(\widetilde{\gamma}_{i}^{\prime}\right)$ for all $i$. Define $w$ and $w^{\prime} \in W$ by

$$
w=s_{\beta_{|\tilde{\gamma}|}} s_{\beta_{|\tilde{\gamma}|-1}} \cdots s_{\beta_{1}} \quad \text { and } \quad w^{\prime}=s_{\delta_{\left|\tilde{\gamma}^{\prime}\right|}} s_{\delta_{\left|\tilde{\gamma}^{\prime}\right|-1}} \cdots s_{\delta_{1}} .
$$

- If $\widetilde{\gamma}=\left(\ldots,-x_{l}, x_{l+1}, x_{l}, \ldots\right)$ then $w=w_{1} s_{l} s_{x_{l}+x_{l+1}} w_{2}$ and $w^{\prime}=$ $w_{1} s_{x_{l}+x_{l+1}} s_{l} w_{2}$ for some $w_{1}, w_{2} \in W$. Hence $w=w^{\prime}$ by $(22)$.

- If $\left(-x_{1}, x_{l+1}\right)$ is not an edge of $\widetilde{\gamma}$, then there exists $h$ and $i>l+1$ so that $w=w_{1} s_{l} s_{x_{l+1} \pm x_{h}} w_{0} s_{x_{l} \pm x_{i}} w_{2}$ and $w^{\prime}=w_{1} s_{x_{l} \pm x_{h}} w_{0} s_{x_{l+1} \pm x_{i}} s_{l} w_{2}$ for some $w_{0}, w_{1}$, and $w_{2} \in W$ such that $w_{0}$ commutes with $s_{l}$. Hence again by $(22)$ we have $w=w_{1} s_{l} s_{x_{l+1} \pm x_{h}} s_{l} w_{0} s_{l} s_{x_{l} \pm x_{i}} w_{2}=$ $w_{1} s_{x_{l} \pm x_{h}} w_{0} s_{x_{l+1} \pm x_{i}} s_{l} w_{2}=w^{\prime}$.

One the other hand, the fact that $\gamma \in \bar{\Sigma}(p, s)$ implies that $\lambda(s)-\lambda(p)=|\gamma|$. Moreover, since $\widetilde{\gamma}^{\prime}$ is an ascending path, Lemmas 5.12 and 6.4 together imply that $\lambda\left(\gamma_{i+1}^{\prime}\right)-\lambda\left(\gamma_{i}^{\prime}\right) \geq 1$ for all $1 \leq i \leq\left|\gamma^{\prime}\right|=|\gamma|$. But this is impossible unless $\lambda\left(\gamma_{i+1}^{\prime}\right)-\lambda\left(\gamma_{i}^{\prime}\right)=1$ for all $i$, which implies that $\gamma^{\prime} \in \bar{\Sigma}(p, s)$.

We are now ready to prove Theorem 6.16

Proof of Theorem 6.16. Let $p=w\left(\mu^{n}\right)$ and $q=w^{\prime}\left(\mu^{n}\right)$. For all $s \in \widehat{\mathcal{O}}_{q}^{T}$, let $\widehat{\alpha}_{s} \in H_{T}^{*}\left(\widehat{\mathcal{O}}_{q} ; \mathbb{Z}\right)$ be the canonical class on the fiber $\widehat{\mathcal{O}}_{q}=\pi^{-1}(\pi(q)) \subset \mathcal{O}_{\mu^{n}}$. Since Proposition 6.8 implies that $\pi$ is a strong symplectic fibration and $\psi_{1}$ is the inclusion, Corollary 5.8, Proposition 6.1 and Lemma 6.2 together imply 
that

$$
\alpha_{p}(q)=\sum_{s \in \widehat{\mathcal{O}}_{q}^{T}}\left(\sum_{\gamma \in \bar{\Sigma}(p, s)} P(\gamma)\right) \widehat{\alpha}_{s}(q) .
$$

If $\gamma \in \bar{\Sigma}(p, s)$ is complete, then $\gamma$ is relevant and $P(\gamma)=Q(\gamma)$. On the other hand, by Lemmas 6.20 and 6.21 , the set of incomplete paths can be decomposed into pairs of paths $\gamma$ and $\gamma^{\prime}$, so that $V\left(\widetilde{\gamma}^{\prime}\right)=V\left(\pi\left(\gamma^{\prime}\right)\right)$ is obtained from $V(\widetilde{\gamma})=V(\pi(\gamma))$ by replacing $x_{k(\gamma)+1}$ by $-x_{k(\gamma)+1}$, where $k(\gamma)=\max \left\{j \mid\left\{-x_{j}, x_{j}\right\} \subset V(\widetilde{\gamma})\right\}$. In particular, $S V(\widetilde{\gamma}) \backslash\left\{-x_{k(\gamma)+1}\right\}=$ $S V\left(\widetilde{\gamma}^{\prime}\right) \backslash\left\{x_{k(\gamma)+1}\right\}$. Additionally, by the definition of $k(\gamma), \pi(s) \neq \pm x_{k(\gamma)+1}$, and so $\eta\left( \pm x_{k(\gamma)+1}, \pi(s)\right)=\pi(s) \mp x_{k(\gamma)+1}$. Hence, by Proposition 6.19

$$
\begin{aligned}
Q(\gamma) & =\pi(s) \prod_{r \in S V(\widetilde{\gamma}) \cap S V\left(\widetilde{\gamma}^{\prime}\right)} \eta(r, \pi(s)) \\
& =\frac{\eta\left(-x_{k(\gamma)+1}, \pi(s)\right)+\eta\left(x_{k(\gamma)+1}, \pi(s)\right.}{2}\left(\prod_{r \in S V(\widetilde{\gamma}) \cap S V\left(\widetilde{\gamma}^{\prime}\right)} \eta(r, \pi(s))\right) \\
& =P(\gamma)+P\left(\gamma^{\prime}\right) .
\end{aligned}
$$

Since $\gamma$ is relevant, but $\gamma^{\prime}$ is not, this implies that

$$
\sum_{\gamma \in \bar{\Sigma}(p, s)} P(\gamma)=\sum_{\gamma \in R(p, s)} Q(\gamma) .
$$

By Propositions 6.5 and 6.9 , this proves part (1) of Theorem 6.16. Finally, by the definition of $S V(\widetilde{\gamma}), \eta(r, \pi(s))$ is a positive root for all $r \in S V(\widetilde{\gamma})$. Hence, if $\gamma$ is complete then $Q(\gamma)=P(\gamma)$ is the product of distinct positive roots and a constant which is either 1 or 2 . On the other hand, if $\gamma$ is incomplete then, by definition of incomplete path, $\pi(s)$ must be a positive root. and so again $Q(\gamma)$ is the product of distinct positive roots.

6.6. Generic coadjoint orbit of type $\boldsymbol{D}_{\boldsymbol{n}}$. The main result of this section is an inductive positive integral formula that expresses the restrictions of the equivariant Schubert classes on a generic coadjoint orbit of type $D_{n}$ in terms of products of distinct positive roots with positive integer coefficients, and the restriction of equivariant Schubert classes on a generic coadjoint orbit of type $D_{n-1}$. To find this formula, we will apply Corollary 5.8 to the natural projection from a generic coadjoint orbit of $S O(2 n)$ to $G r_{2}^{+}\left(\mathbb{R}^{2 n}\right)$, the Grassmannian of oriented two-planes in $\mathbb{R}^{2 n}$ for all $n \geq 4$. (If $n=3$ then a generic coadjoint orbit of type $D_{n}$ is also the complete flag on $\mathbb{C}^{4}$, and so we can use the techniques of Section 6.3.) Despite the fact that $H^{2 n-2}\left(G r_{2}^{+}\left(\mathbb{R}^{2 n}\right) ; \mathbb{R}\right)=\mathbb{R}^{2} \neq \mathbb{R}=H^{2 n-2}\left(\mathbb{C P}^{2 n-2} ; \mathbb{R}\right)$, we will then proceed as in Section 6.5. 
Let $G=S O(2 n), T \subset G$ be a maximal torus, and $W$ be the associated Weyl group; assume $n>1$. We can identify the dual of the Lie algebra of $T$ with $\mathfrak{t}^{*}=\left(\mathbb{R}^{n}\right)^{*}$, so that the set of roots is

$$
R=\left\{ \pm x_{i} \pm x_{j} \mid 1 \leq i<j \leq n\right\} \subset \mathfrak{t}^{*} .
$$

Let $\widehat{G}=S O(2 n-2)$. We can identify the dual of the Lie algebra of a maximal torus $\widehat{T}$ of $\widehat{G}$ with the set of $\left(a_{1}, \ldots, a_{n}\right) \in \mathfrak{t}^{*}$ such that $a_{1}=0$. This identifies the roots of $\widehat{G}$ with the set

$$
\widehat{R}=\left\{ \pm x_{i} \pm x_{j} \mid 2 \leq i<j \leq n\right\} \subset R,
$$

and the Weyl group $\widehat{W}$ of $\widehat{G}$ with the subgroup of $W$ generated by reflections across the roots in $\widehat{R}$; moreover, it induces a map from $H^{*}(B \widehat{T} ; \mathbb{Z})$ to $H^{*}(B T ; \mathbb{Z})$. Equivalently, let $\widetilde{V}=\left\{ \pm x_{1}, \pm x_{2}, \ldots, \pm x_{n}\right\} ; \widehat{W}$ is the kernel of the map $\pi: W \rightarrow \widetilde{V}$ defined by $\pi(w)=w\left(-x_{1}\right)$.

To state our main theorem, we will need several additional definitions. Let

$$
R^{+}=\left\{x_{i} \pm x_{j} \mid 1 \leq i<j \leq n\right\} \subset R
$$

be the set of positive roots. Define

$$
E=\left\{\left(\tau, \tau s_{\beta}\right) \in W \times W \mid l\left(\tau s_{\beta}\right)=l(\tau)+1 \text { and } \beta \in R\right\} .
$$

Given $w$ and $w^{\prime} \in W$, let $\bar{\Sigma}\left(w, w^{\prime}\right)$ denote the set of paths $\gamma=\left(\gamma_{1}, \ldots, \gamma_{|\gamma|+1}\right)$ from $w$ to $w^{\prime}$ in $(W, E)$ such that $\pi\left(\gamma_{i}\right) \neq \pi\left(\gamma_{i+1}\right)$ for all $i$. Equivalently, $\bar{\Sigma}\left(w, w^{\prime}\right)$ is the set of paths from $w$ to $w^{\prime}$ such that each edge is of the form $\left(\tau, \tau s_{\beta}\right)$, where $l\left(\tau s_{\beta}\right)=l(\tau)+1$ and $\beta \in R \backslash \widehat{R}$. Given a sequence $\widetilde{\gamma} \in \widetilde{V}^{k}$, let $V(\widetilde{\gamma})$ be the set of "vertices" of $\widetilde{\gamma}$ :

$$
V(\widetilde{\gamma})=\left\{\widetilde{\gamma}_{1}, \ldots, \widetilde{\gamma}_{k}\right\} \subset \widetilde{V} .
$$

We need the following lemma, which we prove on page 228 .

Lemma 6.22. Given any path $\gamma=\left(\gamma_{1}, \gamma_{2}, \ldots, \gamma_{|\gamma|+1}\right) \in \bar{\Sigma}\left(w, w^{\prime}\right)$, the sequence $\widetilde{\gamma}=\pi(\gamma)=\left(\pi\left(\gamma_{1}\right), \pi\left(\gamma_{2}\right), \ldots, \pi\left(\gamma_{|\gamma|+1}\right)\right)$ is a subsequence of $\left(-x_{1},-x_{2}, \ldots,-x_{n}, x_{n}, \ldots, x_{2}, x_{1}\right)$.

Definition 6.23 A path $\gamma \in \bar{\Sigma}\left(w, w^{\prime}\right)$ with $\pi(\gamma)=\widetilde{\gamma}$ is incomplete if $\left\{-\pi\left(w^{\prime}\right), \pi\left(w^{\prime}\right)\right\} \subset V(\widetilde{\gamma})$. Otherwise $\gamma$ is complete.

Definition 6.24 A path $\gamma \in \bar{\Sigma}\left(w, w^{\prime}\right)$ with $\pi(\gamma)=\widetilde{\gamma}$ is relevant if either it is complete or if it is incomplete and $x_{k(\gamma)+1} \in V(\widetilde{\gamma})$, where ${ }^{7} k(\gamma)=\max \{j \mid$ $\left.\left\{-x_{j}, x_{j}\right\} \subset V(\widetilde{\gamma})\right\}$.

\footnotetext{
${ }^{7}$ Observe that if $\gamma$ is incomplete then by definition $\left\{j \mid\left\{-x_{j}, x_{j}\right\} \subset V(\widetilde{\gamma})\right\} \neq \emptyset$. Moreover, since $\pm x_{i}$ is not a root $\gamma$ cannot contain any edge whose projection is $\left(-x_{n}, x_{n}\right)$, and so-by Lemma $6.22-k(\gamma)<n$.
} 
Finally, given a path $\gamma \in \bar{\Sigma}\left(w, w^{\prime}\right)$, define

$$
P(\gamma)=\widetilde{\Lambda}_{\pi\left(w^{\prime}\right)}^{-}\left(\prod_{i=1}^{|\gamma|} \frac{1}{\pi\left(w^{\prime}\right)-\pi\left(\gamma_{i}\right)} \frac{\pi\left(\gamma_{i+1}\right)-\pi\left(\gamma_{i}\right)}{\eta\left(\gamma_{i}, \gamma_{i+1}\right)}\right),
$$

where $\widetilde{\Lambda}_{\pi\left(w^{\prime}\right)}^{-}$is the product of the $\alpha \in R^{+}$such that $\left\langle\pi\left(w^{\prime}\right), \alpha\right\rangle>0$ and $\pi\left(s_{\alpha}\left(w^{\prime}\right)\right) \neq \pi\left(w^{\prime}\right)$.

The main theorem of this section can be stated as follows.

Theorem 6.25. Fix $n>3$. Let $B \subset G_{\mathbb{C}}$ and $\widehat{B} \subset \widehat{G}_{\mathbb{C}}$ be the Borel subgroups associated to $R^{+}$and $R^{+} \cap \widehat{R}$, respectively, where $G_{\mathbb{C}}$ and $\widehat{G}_{\mathbb{C}}$ are the complexifications of $G=S O(2 n)$ and $\widehat{G}=S O(2 n-2)$, and other symbols are defined as above. Given $w$ and $w^{\prime}$ in $W$, let $K_{w} \in H_{T}^{2 l(w)}\left(G_{\mathbb{C}} / B ; \mathbb{Z}\right)$ be the equivariant Schubert class associated to $w$, and let $\tau \in W$ be the shortest element such that $\pi(\tau)=\pi\left(w^{\prime}\right)$. For all $\widehat{w} \in \widehat{W}$, let $\widehat{K}_{\widehat{w}} \in H_{\widehat{T}}^{2 l(\widehat{w})}\left(\widehat{G}_{\mathbb{C}} / \widehat{B} ; \mathbb{Z}\right)$ be the equivariant Schubert class associated to $\widehat{w}$, and let $R(w, \tau \widehat{w}) \subset \bar{\Sigma}(w, \tau \widehat{w})$ denote the set of relevant paths from $w$ to $\tau \widehat{w}$ in $(W, E)$.

(1) For all $w$ and $w^{\prime}$ in $W$

$$
K_{w}\left(w^{\prime}\right)=\sum_{\widehat{w} \in \widehat{W}}\left(\sum_{\gamma \in R(w, \tau \widehat{w})} Q(\gamma)\right) \tau\left(\widehat{K}_{\widehat{w}}\left(\tau^{-1} w^{\prime}\right)\right),
$$

where for every $\gamma \in R(w, \tau \widehat{w})$

$$
Q(\gamma)= \begin{cases}P(\gamma) & \text { if } \gamma \text { is complete, } \\ P(\gamma) \frac{2 \pi\left(w^{\prime}\right)}{\pi\left(w^{\prime}\right)+x_{k(\gamma)+1}} & \text { if } \gamma \text { is incomplete. }\end{cases}
$$

(2) $Q(\gamma)$ is the product of distinct positive roots for all $\gamma \in R(w, \tau \widehat{w})$.

To prove Theorem 6.25 , we need to translate it into geometrical language. Fix a point

$\mu^{j} \in \mathfrak{t}^{*}$ such that $\mu_{1}^{j}<\cdots<\mu_{j}^{j}<0=\mu_{j+1}^{j}=\cdots=\mu_{n}^{j}$ for each $0 \leq j \leq n$; for simplicity assume that $\mu_{j}^{j}=-1$. Let $\left(\mathcal{O}_{\mu^{j}}, \omega_{j}, \psi_{j}\right)$ be the coadjoint orbit through $\mu^{j}$ for each $j$. The stabilizer of $\mu^{j}$ is

$$
S O(2) \times \cdots \times S O(2) \times S O(2 n-2 j) \quad \text { for all } j ;
$$

in particular, $P_{\mu^{i}} \subseteq P_{\mu^{j}}$ for all $1 \leq j \leq i \leq n$. Moreover, let $\varphi=\psi_{n}^{\xi}: \mathcal{O}_{\mu^{n}} \rightarrow$ $\mathbb{R}$ be a generic component of the moment map that achieves its minimum value at $\mu^{n}$. Observe that, by the definition of $\mu^{n}$, the set $R^{+}$coincides with $\{\alpha \in R \mid(\alpha, \xi)>0\}$. By Proposition 6.1, there exists a canonical class $\alpha_{p} \in H_{T}^{2 \lambda(p)}\left(\mathcal{O}_{\mu^{n}} ; \mathbb{Z}\right)$ for each $p \in \mathcal{O}_{\mu^{n}}^{T}$. The map from $W$ to $\mathcal{O}_{\mu^{n}}^{T}$ given 
by $w \mapsto w\left(\mu^{n}\right)$ identifies the canonical graph of $\mathcal{O}_{\mu^{n}}$ with $(W, E)$; we shall identify these without further comment. Moreover, by Proposition 6.6 and Lemma 6.3, $\Theta\left(r, r^{\prime}\right)=1$ for each edge $\left(r, r^{\prime}\right)$ in $E$.

Let $\pi: \mathcal{O}_{\mu^{n}} \rightarrow \mathcal{O}_{\mu^{1}}$ be the natural projection. Note that $\bar{\Sigma}\left(w, w^{\prime}\right)$ is exactly the set of horizontal paths (with respect to $\pi$ ) from $w$ to $w^{\prime}$; see Definition 5.9. Moreover, given any $\gamma \in \bar{\Sigma}\left(w, w^{\prime}\right)$, the projection $\widetilde{\gamma}=\pi(\gamma)$ is an ascending path in the GKM graph $\left(\widetilde{V}, \widetilde{E}_{\mathrm{GKM}}\right)$ associated to $\left(\mathcal{O}_{\mu^{1}}, \omega_{1}, \psi_{1}\right)$ by Lemmas 5.11 and 5.12 ; this proves Lemma 6.22 . Note that $\left(\widetilde{V}, \widetilde{E}_{\mathrm{GKM}}\right)$ is not a complete graph, because it does not contain the edge $\left(-x_{j}, x_{j}\right)$ for any $j$, but it does contain all other edges. Finally, observe that $\widetilde{\Lambda}_{\pi\left(w^{\prime}\right)}^{-}$is the equivariant Euler class of the negative normal bundle of $\widetilde{\varphi}=\psi_{1}^{\xi}: \mathcal{O}_{\mu^{1}} \rightarrow \mathbb{R}$ at $\pi\left(w^{\prime}\right)$.

Remark 6.26. By Proposition 6.8, the natural projection map $\rho_{j}: \mathcal{O}_{\mu^{j+1}} \rightarrow$ $\mathcal{O}_{\mu^{j}}$ is a strong symplectic fibration with fiber the Grassmannian $P_{\mu^{j}} / P_{\mu^{j+1}} \simeq G r_{2}^{+}\left(\mathbb{R}^{2 n-2 j}\right)$ for all $0 \leq j<n$. However, since $H^{2 n-2 j-2}$ $\left(G r_{2}^{+}\left(\mathbb{R}^{2 n-2 j}\right) ; \mathbb{R}\right)=\mathbb{R}^{2}$, we can not use Theorem 5.5 to express the restriction $K_{w}\left(w^{\prime}\right)$ as a sum of polynomial terms.

To prove the theorem, we need to analyze how the expression of $P(\gamma)$ changes depending on whether $\gamma$ is complete or incomplete.

Proposition 6.27. Let $\gamma=\left(\gamma_{1}, \ldots, \gamma_{|\gamma|+1}\right)$ be a path in $\bar{\Sigma}(p, s)$. Let $\widetilde{\gamma}=$ $\pi(\gamma)$, and let $S V(\widetilde{\gamma})$ be the skipped vertices of $\widetilde{\gamma}$; see Definition 5.23. Then

$$
P(\gamma)=\left\{\begin{array}{cl}
\prod_{r \in S V(\widetilde{\gamma}) \backslash\{-\pi(s)\}} \eta(r, \pi(s)) & \text { if } \gamma \text { is complete, and } \\
\frac{1}{2 \pi(s)} \prod_{r \in S V(\widetilde{\gamma})} \eta(r, \pi(s)) & \text { if } \gamma \text { is incomplete. }
\end{array}\right.
$$

Proof. All the edges $\left(r, r^{\prime}\right) \in \widetilde{E}_{\mathrm{GKM}}$ have magnitude 1; see Definition 5.17. Therefore, since $\Theta\left(r, r^{\prime}\right)=1$ for all $\left(r, r^{\prime}\right) \in E$, the claim follows from an argument similar to the proof of Lemma 5.24.

We are now ready to prove Theorem 6.25.

Proof of Theorem 6.25. Let $p=w\left(\mu^{n}\right)$ and $q=w^{\prime}\left(\mu^{n}\right)$. For all $s \in \widehat{\mathcal{O}}_{q}^{T}$, let $\widehat{\alpha}_{s} \in H_{T}^{*}\left(\widehat{\mathcal{O}}_{q} ; \mathbb{Z}\right)$ be the canonical class on the fiber $\widehat{\mathcal{O}}_{q}=\pi^{-1}(\pi(q)) \subset \mathcal{O}_{\mu^{n}}$. Since Proposition 6.8 implies that $\pi$ is a strong symplectic fibration and $\psi_{1}$ is the inclusion, Corollary 5.8, Proposition 6.1 and Lemma 6.2 together imply that

$$
\alpha_{p}(q)=\sum_{s \in \widehat{\mathcal{O}}_{q}^{T}}\left(\sum_{\gamma \in \bar{\Sigma}(p, s)} P(\gamma)\right) \widehat{\alpha}_{s}(q) .
$$


If $\gamma \in \bar{\Sigma}(p, s)$ is complete, then $\gamma$ is relevant and $P(\gamma)=Q(\gamma)$. On the other hand, Lemmas 6.20 and 6.21 still hold when $G=S O(2 n)$ instead of $S O(2 n+1)$. Indeed, the proof is identical, except that in the proof of Lemma 6.20 we no longer need to consider the case that $\left(-x_{l}, x_{l}\right)$ is an edge of $\widetilde{\gamma}$. Hence, as before, the set of incomplete paths can be decomposed into pairs of paths $\gamma$ and $\gamma^{\prime}$, so that $V\left(\widetilde{\gamma}^{\prime}\right)=V\left(\pi\left(\gamma^{\prime}\right)\right)$ is obtained from $V(\widetilde{\gamma})=V(\pi(\gamma))$ by replacing $x_{k(\gamma)+1}$ by $-x_{k(\gamma)+1}$, where $k(\gamma)=\max \left\{j \mid\left\{-x_{j}, x_{j}\right\} \subset V(\widetilde{\gamma})\right\}$. In particular, $S V(\widetilde{\gamma}) \backslash\left\{-x_{k(\gamma)+1}\right\}=S V\left(\widetilde{\gamma}^{\prime}\right) \backslash\left\{x_{k(\gamma)+1}\right\}$. Additionally, by the definition of $k(\gamma), \pi(s) \neq \pm x_{k(\gamma)+1}$, and so $\eta\left( \pm x_{k(\gamma)+1}, \pi(s)\right)=\pi(s) \mp$ $x_{k(\gamma)+1}$. Hence by Proposition 6.27

$$
\begin{aligned}
Q(\gamma) & =\prod_{r \in S V(\tilde{\gamma}) \cap S V\left(\tilde{\gamma}^{\prime}\right)} \eta(r, \pi(s)) \\
& =\frac{\eta\left(-x_{k(\gamma)+1}, \pi(s)\right)+\eta\left(x_{k(\gamma)+1}, \pi(s)\right)}{2 \pi(s)}\left(\prod_{r \in S V(\tilde{\gamma}) \cap S V\left(\tilde{\gamma}^{\prime}\right)} \eta(r, \pi(s))\right) \\
& =P(\gamma)+P\left(\gamma^{\prime}\right) .
\end{aligned}
$$

By Propositions 6.5 and 6.9, this proves part (1) of Theorem 6.25. The proof of part (2) also proceeds analogously to the proof of Theorem 6.16 (2) in the previous subsection.

\section{References}

[AJS] H.H. Andersen, J.C. Jantzen and W. Soergel. Representations of quantum groups at a pth root of unity and of semisimple groups in characteristic p: independence of p. Astérisque No. 220 (1994).

[B] S.C. Billey, Kostant polynomials and the cohomology ring for $G / B$, Duke Math. J., 96(1) (1999), 205-224.

[Hum] J. Humphreys, Reflection groups and Coxeter groups, Cambridge Studies in Advanced Mathematics, 29, Cambridge University Press, 1990.

[Ki] F. Kirwan, Cohomology of quotients in symplectic and algebraic geometry. Mathematical Notes, Princeton University Press, 1984.

[Kn99] A. Knutson, A Littelmann-type formula for Duistermaat-Heckman measures, Invent. Math., 135(1) (1999), 185-200.

[Kn08] A. Knutson, A compactly supported formula for equivariant localization and simplicial complexes of Bialynicki-Birula decompositions, to appear in Pure App. Math. Q, 6(2) (2010), 501-544.

[Ku] S. Kumar, Kac-Moody groups, their Flag varieties and representation theory, Progress in Mathematics 204. Birkhäuser Boston Inc., Boston, MA, 2002.

[MS] D. McDuff and D. Salamon, Introduction to Symplectic Topology, Oxford Mathematical Monographs, 2004. 
[GHZ] V. Guillemin, T. Holm, and C. Zara, A GKM description of the equivariant cohomology ring of homogeneous space, J. Algebraic Combin., 23(1) (2006), 21-41.

[GSZ] V. Guillemin, S. Sabatini, and C. Zara. Cohomology of GKM fiber bundles, J. Algebr. Combin., 35(1) (2012), 19-59.

[GZ] V. Guillemin and C. Zara, Combinatorial formulas for products of Thom classes, Proceedings of the Geometry, Mechanics, and Dynamics Workshop, 363-405, Springer-Verlag, New York, 2002.

[GKM] M. Goresky, R. Kottwitz, and R. MacPherson, Equivariant cohomology, Koszul duality and the localization theorem, Invent. Math. 131(1) (1998), 25-83.

[GT] R. Goldin and S. Tolman, Towards Generalizing Schubert Calculus in the Symplectic Category, J. Symplec. Geometry, 7(4) (2009), 449-473.

[LT] H. Li and S. Tolman, Hamiltonian circle actions with minimal fixed sets, Int. J. Math. 23(8) (2012), 1250071 (36 pages).

[T] S. Tolman, On a symplectic generalization of Petrie's conjecture, Transactions of the AMS, 362(8) (2010), 3963-3996.

[TW] S. Tolman and J. Weitsman, On the cohomology rings of Hamiltonian T-spaces. Northern California Symplectic Geometry Seminar, 251258, Amer. Math. Soc. Transl. Ser. 2, 196, Amer. Math. Soc., Providence, RI, 1999.

[Za] C. Zara, Positivity of Equivariant Schubert Classes Through Moment Map Degeneration, J. Symplec. Geom., 8(4) (2010), 381-402.

École Polytechnique Fédérale de Lausanne

CH-1015 LAusanne

E-mail address: silvia.sabatini@epfl.ch

UNIVERSITY OF ILLINOIS URBANA-CHAMPAIGN

1409 W. Green Street

URBANA, IL 61801

E-mail address: stolman@math.uiuc.edu

Received 04/13/2010, accepted 07/20/2012

The second author (S.T.) was partially supported by NSF-DMS Grant no. 0707122. The authors would particularly like to thank Victor Guillemin; without his support and encouragement this project would not have been possible. They would also like to thank Catalin Zara for his mathematical insights, and Rebecca Goldin for her support. Finally, we would like to thank the anonymous referee, who made numerous suggestions that improved the exposition. 